(Aus dem pharmakologischen Institut der Universität Greifswald. Geh. Med.-Rat Prof. Dr. H. Schulz.)

\title{
Der Einfluss des Alkohols am Hungertier auf Lebensdauer und Stoffumsatz ${ }^{1}$ ).
}

Von

Privatdozent Dr. med. Martin Kochmann und cand. med. WaIter Hall.

(Mit 21 Textfiguren.)

Die Aufgabe zu untersuchen, ob der Alkohol am Hungertiere Lebensdaver und Stoffumsatz beinflusse, scheint sich auf den ersten Blick in nichts von der bekannten Frage zu unterscheiden, ob der Alkohol ein "Nahrungsmittel" sei oder werigstens ein Nahrungsmittel oder einen Nährstoff vertreten könne. Mit anderen Worten: man könnte der Ansicht sein, dass die gestellte Aufgabe noch einmal von einem anderen Gesichtspunkte aus die Beantwortung der Frage anregen wollte, ob der Alkohol Eiweiss sparen, Kohlehydrate und Fett ersetzen könne. Abgesehen davon, dass diese Frage als gelöst betrachtet werden darf, und zwar im positiven Sinne, so glauben wir, dass die neue Fragestellung sich wesentlich von der früheren unterscheidet. Einmal konnte sich der hungernde Organismus anders verhalten als der genügend ernährte, und dann bot die Aufgabe auch praktisches Interesse, da es vom militärischen und sportlichen Standpunkt aus von Wichtigkeit ist, zu entscheiden, ob der Alkohol beim Fehlen aller Nahrung das Leben wenigstens eine Zeitlang verlängern könne. Dabei waren wir uns von Anfang an darüber klar, dass der Alkohol, in grossen Gaben einverleibt, sicherlich einen schädlichen Einfluss auf Lebensdauer und Stoffumsatz haben würde,

1) Dieses Thema wurde von dem „Verein abstinenter Arzte des deutschen Sprachgebietes, in seiner elften Jahresversammlung vom 6. Oktober 1906 als Aufgabe eines Preisausschreibens gestellt. Die vorliegende Bearbeitung erhielt den ausgesetzten Preis. 
ebenso wie grosse Dosen auf den Blutkreislauf und das Herz, auf Atmung und Nervensystem lähmend wirken müssen.

Wenn nun auch die gestellte Aufgabe sich in wesentlichen Dingen von den Untersuchungen unterscheidet, welche als genügend beantwortet bezeichnet wurden, so steht sie doch immerhin in hinreichend naher Beziehung zu ihnen, so dass es sich wohl verlobnt, einen Blick auf das Material zu werfen, welches zur Beantwortung dieser Frage gedient hat.

Wenn man die Menge der Veröffentlichungen, welche über den Einfluss des Alkohols auf den Organismus erscheinen, mit der Zahl der wirklich experimentellen Arbeiten vergleicht, so sieht man, dass viel zu viel Unberufene, welche gar keine Erfahrung besitzen, sich an der Diskussion beteiligen. Auf dem Gebiete, das uns hier speziell beschäftigt, haben allerdings die wertvollen kritischen Zusammenstellungen, Rosenfeld's ${ }^{1}$ ) und besonders Rosemann's ${ }^{2}$ ), Licht und Klarheit geschaffen; ihre Kritiken sind um so berechtigter und ihre Ansichten, welche sie über den Einfluss des Alkohols auf den Stoffumsatz haben, um so begründeter, als sie selbst zur Klärung der Frage durch eine Reihe eigener experimenteller Arbeiten wesentlich beigetragen haben. Es wäre unnätz, hier in extenso das zu wiederholen, was die beiden genannten Forscher in ihren ausführlichen Mitteilungen schon niedergelegt und trotz strengster Kritik als richtig erkannt haben. Wir können uns aus diesen Gründen darauf beschränken, die Resultate der experimentellen Tatsachen in aller Kürze wiederzugeben, wobei wir den Darlegungen der beiden genannten Autoren folgen.

Wie Rosemann überzeugend nachgewiesen hat, befinden sich fast alle experimentellen Arbeiten, welche sich einwandfreier Methoden bedienten, in erfreulicher Übereinstimmung, so dass sich aus der grossen Zahl mühseliger Untersuchungen folgendes als Ergebnis herauskristallisieren lässt.

1) G. Rosenfeld, Der Einfluss des Alkohols auf den Organismus. Wiesbaden 1901.

2) R. Rosemann, Der Einfluss des Alkohols auf den Eiweissstoffwechsel. Zusammenfassende kritische Darstellung nebst Mittheilung neuerer Stoffwechselversuche am Menschen. Sep.-Abdr. a. d. Arch. f. d. ges. Physiol. Bd. 86 S. 307. 1901. - Derselbe, Der Einfluss des Alkohols auf den Eiweissstoffwechsel. Nachtrag zu der zusammenfassenden kritischen Darstellung in Bd. 86 d. Arch. f. d. ges. Physiol, Bd. 94 S. 557. 
Der in den Organismus eingeführte Alkohol wird, wie $\mathrm{Binz}^{1}$ ) und seine Schüler Heubach ${ }^{2}$, Schmidt ${ }^{3}$ ), Bodländer ${ }^{4}$ ) nachweisen konnten, zum weitaus grössten Teile verbrannt. Der letztgenaunte Autor zeigte, dass nur 3\% der eingeführten Alkoholmengen. in nicht oxydiertem Zustande ausgeschieden werden. Strassmann ${ }^{5}$ ) sowie Atwater ${ }^{6}$ ) und Benedict ${ }^{7}$ ) konnten im wesentlichen die Resultate der Binz'schen Schule bestätigen, ebenso wie neuerdings Goddard ${ }^{8}$ ), so dass man annehmen kann, dass mindestens $90 \%$ bis $95 \%$ des eingeführten Alkohols im Organismus der Oxydation zu Wasser und Kohlensäure anheimfallen. Andererseits konnten dann Geppert ${ }^{9}$ ) und $\mathrm{Zuntz}^{10}$ ) in Gemeinschaft mit Berdez am Menschen in exaktester Weise zeigen, dass die $\mathrm{CO}_{2}$-Ausscheidung und die O-Aufnahme nach Einverleibung von Alkohol keine in Betracht kommenden Veränderungen erleide. Der Alkohol musste infolgedessen auf Kosten anderer Substanzen, nämlich der Kohlehydrate und des Fettes, oxydiert worden und diese von der Verbrennung verschont geblieben sein. Dann konnte von einer ganzen Anzahl von Autoren, unter denen Mogilianski ${ }^{11}$ ), Neumann ${ }^{12}$ ), Atwater

1) C. Binz, Die Ausscheidung des Weingeistes durch Nieren und Lunge. Nach den Versuchen der Herren H. Heubach und A. Schmidt. Arch. f. exper. Path. u. Pharm. Bd. 6 S. 287. 1877.

2) H. Heubach, Inaug.-Diss. Bonn 1875. - Quantitative Bestimmung des. Alkohols im Harn. Arch. f. exper. Path. u. Pharm. Bd. 8 S. 446. 1878.

3) A. Schmidt, Inaug.-Diss. Bonn 1876.

4) G. Bodländer, Die Ausscheidung aufgenommenen Weingeistes aus dem Körper. Arch. f. d. ges. Physiol. Bd. 32 S. 398. 1883.

5) Fr. Strassmann, Untersuchungen über den Nährwerth und die Ausscheidung des Alkohols. Arch. f. d. ges. Physiol. Bd. 49 S. 315. 1891.

6) u. 7) Atwater and Benedict, Experiments on the metabolism of matter and energy in the human body. Bulletin no. 69 U.S. Department of agriculture, Office of experiments stations. Washington 1899.

8) W. H. God dard zeigt allerdings, dass nur mässige Quantitäten des eingeführten Alkohols zu $95 \%$ oxydiert werden, sehr grosse Mengen dagegen würden zu $50 \%$ unverändert ausgeschieden. Alcohol as a food. The Lancet vol. II p. 1132.1904 .

9) J. Geppert, Die Einwirkung des Alkohols auf den Gaswechsel des Menschen. Arch. f. exper. Path. u. Pharm. Bd. 22 S. 367. 1887.

10) N. Zuntz, Beitrag zur Kenntnis der Einwirkung des Weingeistes anf den Respirationsprozess des Menschen. Fortschr. d. Med. 1887 S. 1.

11) A. M. Mogilianski, Materialien zur Diätetik des Alkohols. Der Einfluss des Alkohols auf die Assimilation und den Stoffwechsel des Stickstoffes und die Assimilation der Fette. Inaug.-Diss. St. Petersburg 1889.

12) R. O. Neumann, Über die eiweisssparende Kraft des Alkohols. Neue Stoffwechselversuche am Menschen. Vorläufige Mittheilung. Münch. med. Wochenschrift Bd. 48 S. 1126.1901. 
und Benedict ${ }^{1}$ ), Rosenfeld-Chotzen ${ }^{2}$ ), Clopatt ${ }^{3}$ ), Offer ${ }^{4}$ ) und Rosemann ${ }^{5}$ ) genannt werden müssen, konstatiert werden, dass der Alkohol ähnlich wie Fett Eiweiss sparen könne. Von Rosemann wurden in seiner kritischen Zusammenfassung wohl auch alle Ursachen angegeben, welche einzelne Autoren $\mathrm{zu}$ anderen Resultaten kommen liessen, sei es, dass die Methoden nicht exakt genug waren, wie bei dem ersten Versuch Offer's, oder dass die Versuche nicht lange genug durchgeführt wurden [Miura $\left.\left.{ }^{6}\right)\right]$. Die Eiweisssparung durch Alkohol tritt, wie Atwater und Benedict ${ }^{7}$ ) und besonders Rosemann zeigen konnten, erst nach einiger Zeit ein, während nach Beginn der Alkoholdarreichung ein vermehrter Eiweisszerfall oder doch wenigstens eine vermehrte N-Ausscheidung stattfindet, was auf einen toxischen Einfluss des Alkohols bezogen wird, der durch Gewöhnung aber bald wieder vorübergeht. Bei allen Arbeiten fällt es übrigens auf, dass mit ganz ausserordentlich hohen Alkoholgaben gearbeitet wurde, und dies noch zum Teil bei Leuten, welche an Alkohol gar nicht oder nur wenig gewöhnt waren. Übrigens macht auch Rosemann schon darauf aufmerksam, dass manche Verschiedenheiten in den Versuchsergebnissen auf diesen Umstand zurückzuführen wären. Es verlohnt sich schon, einen Augenblick bei den Quantitäten eingeführten Alkohols zu verweilen. Mogilianski ${ }^{8}$ ) gibt $60-140 \mathrm{cem}$ Alcohol absolutus, im Durchschnitt also $100 \mathrm{cem}$, Stammreich $\left.{ }^{9}\right) 70,4 \mathrm{~g}$, Miura $\left.{ }^{10}\right) 65 \mathrm{~g}$,

1) Atwater and Benedict, l. c.

2) Rosenfeld-Chotzen, Der Alkohol als Nahrungsmittel. Therapie der Gegenwart. Februar 1900.

3) A. Clopatt, Über die Einwirkung des Alkohols auf den Stoffwechsel des Menschen. Skandinav. Arch. f. Physiol. Bd. 11 S. 354. 1901.

4) Th. R. Offer, Inwiefern ist Alkohol ein Eiweisssparer? Wiener klin. Wochenschr. Bd. 12 Nr. 41 S. 1009.1899.

5) Rosemann, l. c.

6) K. Miura, Über die Bedeutung des Alkohols als Eiweisssparer in der Ernährung des gesunden Menschen. Zeitschr. f. klin. Medizin Bd. 20 s. 1. 1892.

7) Atwater and Benedict, Memoirs of the national acad. of sciences. vol. 8. Sixth memoir. Washington 1902.

8) Mogilianski, l. c.

9) M. Stammreich, Über den Einfluss des Alkohols auf den Stoffwechsel des Menschen. Inaug.-Diss. Berlin 1891.

10) Miura, l. c.

E. Pflüger, Archiv für Physiologie. Bd. 127. 
Strøm $\left.{ }^{1}\right) 52-86 \mathrm{~g}$, Schmidt $\left.{ }^{2}\right) 80-120 \mathrm{~g}$, Schöneseiffen ${ }^{3}$ ) $135 \mathrm{~g}$, Neumann ${ }^{4}$ ) $100 \mathrm{~g}$, Atwater und Benedict $\left.{ }^{5}\right) 72,5 \mathrm{~g}$, Rosenfeld-Chotzen ${ }^{6}$ ) 120 g, Bjerre ${ }^{7}$ ) 167,6 g, Clopatt 8 ) 87,04 g, Rosemann ${ }^{9}$ ) 143,5 g, Offer ${ }^{10}$ ) $100 \mathrm{~g}$. Das sind sicher ganz erhebliche Mengen. Auf Rot- oder Rheinwein von 10\% Alkoholgehalt umgerechnet, bedeutet das Mengen von durchschnittlich 1,5 Liter, Mengen, welche zum Teil an Alkohol ungewohnte Personen verabreicht wurden. Und noch eins ist zu bedenken: Nicht alle Versuche wurden wie die Rosemann's mit Wein, welcher $10 \%$ Alkohol enthielt, angestellt, die meisten nahmen ihn viel konzentrierter zu sich, sei es als einfache hochkonzentrierte Alkohollösung, sei es als Kognak, Whisky und ähnlichem mehr. Wer sich mit pharmakologischen Problemen jemals beschäftigt hat, weiss, wieviel die Konzentration zur Wirkung beiträgt, indem einerseits die Resorption durch die steigende Konzentration verändert werden kann, andererseits auch auf einmal grössere Massen zur Wirksamkeit gelangen. Die verabreichten Mengen Alkohols müssen besonders dann als grosse imponieren, wenn man sie mit jenen vergleicht, durch welche $\mathrm{Koch}$ $\operatorname{man} n^{11}$ ) beim gesunden Menschen schon den Blutkreislauf im Sinne einer begünstigenden Wirkung beeinflussen konnte, Versuche, welche von Bachem ${ }^{12}$ ) nachgeprüft und bestätigt werden konnten. 40 bis

1) H. Str $\emptyset \mathrm{m}$, Aethylalkohols Indvirkning paa Kvaelstofomsaetningen. Inaugural-Dissertation. Kjøbenhavn 1894.

2) E. Schmidt, Über den Einfluss des Alkohols auf den Eiweissstoffwechsel des menschlichen Körpers. Inang.-Diss. Greifswald 1898.

3) 0. Schöneseiffen, Über den Wert des Alkohols als eiweisssparendes Mittel. Inaug.-Diss. Greifswald 1899.

4) R. O. Neumann, Die Bedeutung des Alkohols als Nahrungsmittel, Arch. f. Hygiene Bd. 36 S. 1. 1899.

5) Atwater and Benedict, l. c.

6) Rosenfeld-Chotzen, 1. c.

7) P. Bjerre, Über den Nährwerth des Alkohols. Skandinav. Arch. f. Physiol. Bd. 9 S. 323. 1900.

8) Clopatt, l. c.

9) Rosemann, l. c.

10) Offer, 1. c.

11) M. Kochmann, Experimentelle Beiträge zur Wirkung des Alkohols auf den Blutkreislauf des Menschen. Arch. internat. de Pharmacodynamie et de Thérapie t. 15 p. 443.1905.

12) C. Bachem, Über den Einfluss kleinerer Mengen alkoholischer Getränke auf den Blutdruck des Menschen. Arch. f. d. ges. Physiol. Bd. 114 S. 508. 1906. 
$100 \mathrm{ccm} 10 \%$ igen Alkohols genügten schon, um eine Blutdrucksteigerung zu erzeugen, und diese Mengen variierten, je nachdem es sich um alkoholgewöhnte oder abstinente Individuen handelte. Dass der Alkohol in den grossen Gaben toxisch wirken würde, ergab sich in manchen der Versuche aus der Störung des subjektiven Befindens; so wird berichtet, dass Dr. Chotzen nach der Alkoholaufnahme "verkatert" gewesen sei. Es verlohnte sich vielleicht, die Versuche mit wesentlich geringeren Alkoholmengen am Menschen anzustellen, und es wäre nicht ausgeschlossen, dass alsdann eine schädigende Wirkung des Alkohols auf den Eiweissstoffwechsel selbst in den ersten Tagen nicht stattfindet. In neuester Zeit hat Rosenfeld $^{1}$ ) von Walter und Pringsheim²) die Frage nach der Eiweisssparung durch Alkohol wieder aufnehmen und durch Versuche am Menschen (Selbstversuch) zeigen lassen können, dass der Alkohol besser als Kohlehydrate das Körpereiweiss schont. Diese Erscheinung tritt, was besonders interessant ist, noch bei Gaben auf, welche die Intelligenzleistungen, die Muskelkraft und das Herz schon erheblich schädigen. Es wird auch besonders betont, dass der Alkohol als Zugabe zu einer genügenden Kost gleich von Anfang an den Eiweissbestand schone und dies nicht erst nach einer Periode vermehrter $N$-Ausfuhr (Rosemann) bewerkstellige.

Auf den Unterschied, welchen kleinere und grössere Alkoholgaben in der Wirkung auf den N-Stoffwechsel haben können, hat $\mathrm{Munk}^{3}$ ) in seinen Versuchen am Hunde hingewiesen, wenn er sah, dass Gaben von $1-1,5 \mathrm{ccm}$ Alcohol absolutus pro Kilogramm Tier eine Eiweisssparung erzeugten, grössere Gaben aber eine Eiweissschädigung veranlassten. Bei den Versuchen Chauveau's ${ }^{4}$ ) am Hunde wurden, wie Rosemann mit Recht hervorhebt, ganz enorme Dosen von Alkohol gegeben, so dass man schon aus diesem Grunde gegen eine Verallgemeinerung der Ergebnisse dieser experimentellen Arbeit Einspruch erheben darf und muss.

1) G. Rosenfeld, Der Alkohol als Nahrungsmittel. Centralblatt f. innere Med. Heft 12. 1906.

2) J. Pringsheim, Alkohol und Eiweissstoffwechsel. Selbstversuch. Zeitschrift f. diät. u. physik. Therapie Bd. 10 S. 274. 1906.

3) J. Munk, Der Einfluss des Alkohols und des Eisens auf den Eiweirsstoffwechsel. Arch. f. Anat. u. Physiol. 1879; zit. nach Ros enfeld.

4) Chaureau (zit. nach Rosemann). Compt. rend. t. 132 p. 65-70 et p. $110-114.1901$. 
Die sonstigen Versuche, welche an Tieren angestellt wurden, sind nicht ganz einwandfrei, und wir können es uns versagen, auf sie näher einzugehen. Weiske und Flechsig ${ }^{1}$ ) zeigten durch ihre Versuche, dass beim Hammel eine eiweisssparende Wirkung des Alkohols nicht zu bemerken sei; wenn übrigens vom Hammel auf alle Herbivoren geschlossen wird, so glauben wir, dass dabei zu weit gegangen wird, da der Hammel in gewisser Beziehung einen anderen Stoffwechsel hat wie z. B. ein Kaninchen; es soll dabei nur erinnert werden, dass der Hammel nach Weber ${ }^{2}$ ) nur Spuren von Phosphorsäure im Urin ausscheidet, während das Kaninchen sich ähnlich wie der Hund verhält.

Die Versuche am kranken Menschen von Diakonow ${ }^{3}$ ) sind nicht einwandfrei genug, wie Rosemann hervorhebt, als dass man bindende Schlüsse daraus ziehen dürfte, und ebensowenig beweisen die Stoffwechselversuche Hirschfeld' $\mathrm{s}^{4}$ ) bei Diabetikern mit Sicherheit eine günstige Wirkung des Alkohols im Sinne einer Eiweisssparung, wenn man auch zugeben muss, dass eine solche höchstwahrscheinlich vorliegt.

Sehr interessant sind die Versuche von Benedict und Törö $\mathrm{k}^{5}$ ), die fanden, dass der Alkohol nicht nur die Ausscheidung des Acetons beim kohlehydratfrei ernährten Gesunden, sondern besonders auch beim Diabetiker vermindere; wenn auch beim Diabetiker infolge der Alkoholdarreichung der Zuckerumsatz herabgesetzt. werde, so dass die Zuckerausscheidung zurückgeht, so wird die Verminderung des Acetons doch darauf zurückgeführt, dass ein Teil des „acetonbildenden Fettes" durch nichtacetonbildenden Alkohol ersetzt wird. Besser als Kohlehydrate und Fett vermag nach den genannten

1) Weiske u. Flechsig, Versuche über die Wirkung von Alkoholaufnahme bei Herbivoren. Journ. f. Landwirtsch. Bd. 34 S. 153, 1886 u. Bd. 37 S. 327.1889 .

2) S. W eber, Versuche über künstliche Einschränkung des Eiweissumsatzes bei einem fiebernden Hammel. Arch. f. exper. Path. u. Pharm. Bd. 47 S. 19.1902.

3) D. J. Diakonow, Zur Frage nach dem Einfluss des Alkohols auf die Assimilation und den Stoffwechsel des Stickstof's beim Typhus. Inaug.-Diss. St. Petersburg 1890.

4) F. Hirschfeld, Die Anwendung des Alkohols bei der Zuckerharnruhr. Berl. klin. Wochenschr. Bd. 32 s. 95.1895.

5) H.'Benedict und B. Török, Der Alkohol in der Ernährung der Zuckerkranken. Experimentelle Untersuchung. Zeitschr. f. klin. Mediz. Bd. 60 S. 329. 1906. 
Autoren der Alkohol den Eiweissbestand des Organismus der Diabetiker zu schonen.

Versuche, experimentell zu bestimmen, ob auch im fiebernden Organismus bei Ersatz von $100 \mathrm{~g}$ Zucker durch $60 \mathrm{~g}$ Alkohol in $35 \%$ Lösung Eiweiss gespart werde, fielen hei einwandfreier Methode in bejahendem Sinne aus [Ott $\left.\left.{ }^{1}\right)\right]$. Der Alkohol war, ohne eine toxische Wirkung zu entfalten, in vollem Umfange für die Kohlehydrate eingetreten.

In direktem Zusammenhange mit unserer Aufgabe stehen die Arbeiten, welche sich mit der Einwirkung des Alkohols auf den Stoffwechsel des hungernden Mensehen befassen. Romey ${ }^{2}$ ) gab seinen Versuchspersonen 48-63 Stunden nur Wasser zu trinken, entzog ihnen aber sonst vollkommen die Nahrung. Einige Zeit nach Beginn des Versuches wurde ihnen dann eine bestimmte Menge Alkohols (30-51 cem mit ungefähr $100 \mathrm{ccm}$ Wasser vermischt) gereicht. Wenn nun auch nach der Alkoholaufnahme keine Zeichen wirklicher Trunkenheit auftraten, so waren bei den Versuchspersonen doch immer Depressionserscheinungen und Brechneigung zu konstatieren, der in einigen Fällen auch nachgegeben wurde. Trotz mancher Einwände, welche Rosemann gegen die Versuche erhebt, ist doch aus ihnen mit Sicherheit $z u$ entnehmen, dass bei den immerhin erheblichen Alkoholmengen, welche zur Verwendung gelangten, von einer eiweisssparenden Wirkung des Alkohols nicht die Rede sein konnte; allerdings kann auch nicht mit viel mehr Recht von einem toxischen Einfluss gesprochen werden.

Rosemann stellte ebenfalls mehrere Versuche an, die im folgenden wörtlich angeführt sein mögen, weil sie wohl in jeder Beziehung den berechtigten Anforderungen entsprechen: „Bei meinen eigenen Versuchen nahm ich Rücksicht auf die (oben) bereits mitgeteilten Resultate der reinen Hungerversuche, bei denen sich herausgestellt hatte, dass die Stickstoffausscheidung des Hungernden am zweiten Tage schon an und für sich eine ausgesprochene Tendenz zur Steigerung besitzt. Dagegen zeigt die Stickstoffausscheidung am Nachmittage des ersten Hungertages im allgemeinen ein gleichmässiges

1) A. Ott, Über den Einfluss des Alkohols auf den Eiweissstoffwechsel bei Fiebernden. Arch. f. exper. Path. u. 'Pharm. Bd. 47 S. 267. 1902.

2) Romeyn, Onderzoekingen over den invloed van Alkohol op den mensch. Academisch Proefschrift. Nijmegen 1887. 
'Abfallen, und es ist somit diese Zeit am geeignetsten zur Ausführung der Alkoholversuche. Im ganzen wurden drei Versuche mit Alkohol am hungernden Menschen angestellt. Die Versuchsanordnung war stets die gleiche. Die letzte Mahlzeit wurde am Abend des vorhergehenden Tages um $7 \mathrm{Uhr}$ genommen. Alle zwei Stunden, mit Ausnahme der Nacht, wurden $150 \mathrm{ccm}$ Wasser getrunken, um ein Zurückbleiben von Stoffwechselendprodukten infolge mangelhafter Diurese nach Möglichkeit zu vermeiden. Der Harn wurde stets in zweistündigen Perioden gesammelt, mit Ausnahme der Zeit von 12 Uhr Abends bis 7 Uhr Morgens, in welcher der Gesammturin aufgefangen wurde. Die Stickstoffbestimmung geschah nach Kjeldahl. Am Nachmittag des ersten Hungertages wurden sodann $50 \mathrm{ccm}$ Alkohol von 96 Vol. $\%$ mit $100 \mathrm{ccm}$ Wasser vermischt getrunken. Besonders auffällige Symptome der Alkoholwirkung kamen nur bei dem letzten Versuche vor, bei welchem sowohl um 1 Uhr als auch um 5 Uhr Nachmittags je $50 \mathrm{ccm}$ Alkohol genommen wurden. Während die erste Dosis ohne weitere besondere Symptome vertragen wurde, stellten sich im Anschluss an die zweite Alkoholaufnahme höchst unangenehme, collapsähnliche Erscheinungen ein: allgemeines, sehr starkes Unbehagen, schwacher Puls, starker, kalter Schweiss; das Gehen wurde der Versuchsperson schwer. Diese Symptome verschwanden wieder von selbst, nachdem die Versuchsperson sich eine Zeit lang ruhig verhalten hatte. Alles Weitere ergeben die folgenden Tabellen.

\begin{tabular}{l|l|l|l|l}
\hline Harnmenge & Prozent-N & Gesamt-N & $\begin{array}{c}\text { Alkohol } \\
\text { ccm }\end{array}$ \\
\hline
\end{tabular}

Versuch IV. Versuchsperson R.

$7-9 \mathrm{~h}$ vorm.
$9-11 \mathrm{~h} "$
$11-1 \mathrm{~h} "$
$1-3 \mathrm{~h}$ nachm.
$3-5 \mathrm{~h}$ "
$5-7 \mathrm{~h}$ "
$7-9 \mathrm{~h}$ abends
$9-11 \mathrm{~h} "$

\begin{tabular}{|r}
89 \\
314 \\
420 \\
242 \\
814 \\
94 \\
87 \\
69
\end{tabular}

0,9492
0,4284
0,2604
0,4592
0,1386
0,8064
0,8288
1,1004

0,8484
1,3452
1,0937
1,1113
1,1282
0,7580
0,7211
0,7593

Versuch V, Versuchsperson L.

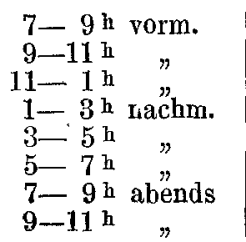

69
108
207
43
36
400
85
36




\begin{tabular}{l|l|l|l|l}
\hline \hline & Harnmenge & Prozent-N & Gesamt- $\mathrm{N}$ & $\begin{array}{c}\text { Alkohol } \\
\mathrm{ccm}\end{array}$ \\
\hline
\end{tabular}

Versuch VI. Versuchsperson L.

\begin{tabular}{|c|c|c|c|}
\hline $\begin{array}{l}7-9 \mathrm{~h} \text { vorm. } \\
9-11 \mathrm{~h} \quad " \\
11-1 \mathrm{~h} \text { " } \\
1-3 \mathrm{~h} \text { nachm. } \\
3-5 \mathrm{~h} \quad " \\
5-7 \mathrm{~h} \quad " \\
7-9 \mathrm{~h} \text { abends } \\
9-11 \mathrm{~h} \quad "\end{array}$ & $\begin{array}{r}57 \\
144 \\
84 \\
368 \\
157 \\
510 \\
77 \\
85\end{array}$ & $\begin{array}{l}1,6340 \\
0,6104 \\
0,8848 \\
0,2464 \\
0,4632 \\
0,1820 \\
1,0722 \\
1,1986\end{array}$ & $\begin{array}{l}0,9314 \\
0,8790 \\
0,7432 \\
0,9068 \\
0,7273 \\
0,9282 \\
0,8256 \\
1,0188\end{array}$ \\
\hline
\end{tabular}

In Versuch IV zeigt sich eine nur geringfügige Wirkung des Alkohols, indem die Stickstoffausscheidung von 1,1113 in der Periode $\mathrm{L}-3^{\mathrm{h}}$, auf 1,1282 in der Periode $3-5^{\mathrm{h}}$ steigt. Wenn man aber berücksichtigt, dass sonst in der Periode $3-5$ Uhr die Stickstoffausscheidung regelmässig niedriger ist als in der Periode $1-3 \mathrm{~h}$, so ergibt sich, dass auch in diesem Versuche im Anschluss an die Alkoholaufnahme eine Erhöhung der Stickstoffausscheidung eingetreten ist. Ganz ausgesprochen ist dies in Versuch V und VI. Doch erstreckt sich regelmässig die Steigerung der Stickstoffausscheidung gerade nur auf die zweistündige Periode, in welcher der Alkohol genommen wurde; in der nächsten Periode ist regelmässig die Sticksoffausscheidung schon wieder niedriger.

Es ergibt sich also aus diesen Versuchen, in Übereinstimmung mit mehreren Versuchen Romeyn's, dass im Anschluss an die Alkoholaufnahme beim Hungernden eine allerdings sehr geringfügige Erhöhung der Stickstoffausscheidung eintritt. Für die Erklärung dieser Erscheinung wird man zunächst auf die vermebrte Diurese Bezug nehmen müssen, die unter diesen Verhältnissen in der Mehrzahl der Versuche eine sehr starke Zunahme zeigt. Es fragt sich aber, ob die Steigerung der Stickstoffausscheidung ganz auf Rechnung der Diurese gesetzt werden kann. Ich habe, um darüber Aufschluss zu erhalten, den Versuch VI angestellt, in welchem eine zweimalige Alkoholaufnahme um 1 Uhr und um 5 Uhr Nachmittags stattfand. Wenn die Erhöhung der Stickstoffausscheidung nach der ersten Alkoholzufuhr als eine Ausspülung liegen gebliebener Stoffwechselendprodukte aufzufassen war, so hätte man erwarten sollen, dass bei der zweiten Alkokolzufuhr die Steigerung der Stickstoffausscheidung ausbleiben oder wesentlich geringer hätte werden müssen, da ja durch die erste Alkoholzufuhr bereits eine Ausspülung des Körpers 
stattgefunden hatte. Wie man sieht, ist aber die Erhöhung der Stickstoffausscheidung im Anschluss an die zweite Alkoholaufnahme sogar noch grösser als die nach der ersten. Ich glaube daher nicht, dass die Vermehrung der Stickstoffausscheidung einzig und allein durch die Diurese bedingt ist, sondern dass wir es vielmehr mit einer direkten Wirkung des eingeführten Alkohols auf die Eiweisszersetzung des Hungernden zu tun haben. Es bleibt aber weiterhin zweifelhaft, ob diese Wirkung dem Alkohol allein zukommt, oder ob nicht überhaupt die Einführung jedes Nahrungsmittels in den leeren Magendarmkanal des Hungernden eine derartige geringfügige Erhöhung der Stickstoffausscheidung veranlassen würde. Ich babe daher zum Vergleich noch die folgenden Hungerversuche angestellt, bei denen am Nachmittage des ersten Hungertages $70 \mathrm{~g}$ Zucker resp. $35 \mathrm{~g}$ Butter, die $50 \mathrm{ccm}$ Alkohol äquivalenten Mengen, natürlich ohne jede weitere Zugabe, aufgenommen wurden." (Folgen in der Arbeit Rosemann's die Tabellen.)

"In Versuch VII hatte die Aufnahme des Zuckers keinen Einfluss auf die Stickstoffausscheidung. Dagegen ist ein solcher deutlich vorhanden in Versuch VIII und IX. Die Einführung der Butter (Versuch X) hatte wieder keine Wirkung. Das letztere negative Re. sultat lässt sich vielleicht so erklären, dass die Resorption der Butter längere Zeit in Anspruch nimmt, während der Zucker, ebenso wie der Alkohol, sehr viel schneller zur Aufnahme kommt. Weshalb in Versuch VII nach dem Zucker eine Wirkung ausgeblieben ist, lässt sich natürlich nicht sagen. So viel geht jedenfalls aus Versuch VIII und IX deutlich hervor, dass auch nach Einführung von Zucker, also einem verhältnismässig indifferenten Stoffe, eine Erhöhung der Stickstoffausscheidung des Hungernden eintreten kann. Ich glaube daher, dass die Steigerung der Stickstoffausseheidung beim Hungernden durch den Alkohol nichts für diesen Charakteristisches hat, und dass daher auf dieselbe ein wesentliches Gewicht nicht zu legen ist."

Das einzige, was auch bei diesen Versuchen Rosemann's zu bemängeln wäre, würden die verhältnismässig grossen Alkokolgaben sein, welche ja auch wenigstens in dem einen Fall zu ganz erheblichen Störungen des subjektiven und objektiven Befindens geführt haben. Die Alkoholgaben müssen auch deshalb schon als grosse bezeichnet werden, weil die Konzentration ungefähr $30 \%$ betrug, also dem Alkoholgehalt guten Kognaks entsprach, und sie in den hungernden 
und deshalb weniger widerstandsfähigen Organismus eingeführt wurden. Man bedenke nur, was es heisst, 1-1/2 Weinglas eines starken Schnapses auf nüchternen Magen zu sich zu nehmen oder gar, wie in den Versuchen Rosemann's, 20 Stunden nach der letzten Mahlzeit. Wenn bei einer solchen Dosierung des Alkohols toxische Effekte erreicht werden, so ist das eigentlich nicht wunderbar, und es spricht für die relative Unschädlichkeit einer e inma ligen grösseren Dosis, wenn in den Versuchen Rosemann's eine toxische Wirkung nicht zu bemerken war.

Es versteht sich von selbst, dass man die Versuche am Menschen, welche die Frage nach der Wirkung des Alkohols auf den Stoffumsatz beantworten sollten, nicht bis zum Äussersten forsetzen konnte, was gleichbedeutend wäre mit der Lösung unserer Aufgabe, ob der Alkohol die Lebensdauer des hungernden Organismus im positiven oder negativen Sinne beeinflussen könne. Die Antwort auf diese Frage mussten Tierversuche bringen, und über diese soll in extenso in folgendem berichtet werden. Doch vorher wollen wir noch Umschau halten, was sonst über den Einfluss des Alkohols auf Stoffwechselvorgänge bekannt ist.

Die Harnsäureausscheidung unter dem Einfluss des Alkohols ist verschiedentlich der Gegenstand experimenteller Untersuchungen geworden. Doch sind die Ergebnisse keineswegs derart beschaffen, dass daraus eindeutige Schlüsse gezogen werden könnten. Es liegen sowohl Versuche am Hund wie am Menschen vor, die aber in Anbetracht des gerade bezüglich der Harnsäure und der Purinkörper verschiedenen Verhaltens des Hundes und des Menschen kaum verglichen werden können. Donogány und Tibáld ${ }^{x}$ ) fanden, dass kleinere Alkoholgaben die Harnsäureausscheidung vergrössern, während grössere Dosen sie unverändert lassen. Chittenden ${ }^{2}$ ) zeigte, dass bei Hunden nach allerdings erheblichen Alkoholgaben (1-4 ccm Alkohol pro Kilogramm Tier) eine Vermehrung der Harnsäure auftritt.

Die Versuche am Menschen geben ein sehr wechselndes Bild:

1) Donogány und Tibáld, Einfluss des Alkohols auf den Eiweisszerfall. Ungar. Arch. f. Med. Bd. 3. S. 189. 1895.

2) R. H. Chittenden, The influence of Alcohol on Proteid metabolism. The journ. of Physiology vol. 12 p. 220. Cambridge 1891. 
[Herter und $S \operatorname{mith}^{1}$ ), Leber ${ }^{2}$ )], bald ein Gleichbleiben, bald ein Ansteigen [Hermann ${ }^{3}$ ), Rosenfeld ${ }^{4}$ )], bald wieder einen Rückgang der Harnsäureausscheidung [Leber, Haeser $\left.\left.{ }^{5}\right)\right]$. Auch hier sind die Alkoholgaben recht versehiedene und vor allen Dingen als sehr hohe zu bezeichnen. Dann aber sind die Abweichungen der ausgeschiedenen Harnsäuremengen während der Alkoholperiode auch bei den methodisch einwandfreien Versuchen im Verhältnis zu den normalen Schwankungen $\left.\left[\mathrm{Tomaschn}{ }^{6}\right)\right]$ so kleine, dass irgendwelche weitgehende Schlussfolgerungen nicht gezogen werden können.

Auch wenn man den nur wenig Schwankungen aufweisenden Verlauf der Harnsäureausscheidung beim Hungernden unter dem Einfluss des Alkohols verfolgt, ist doch keine erhebliche Veränderung in quantitativer und qualitativer Hinsicht zu beobachten [Rosemann $\left.{ }^{7}\right)$. Rosenfeld glaubte aus der geringen Vermehrung der Harnsäure bei gleichzeitiger Verminderung der Stickstoffausfuhr ab•eiten zu können, dass die Nukleoalbumine zerstört, während die anderen Eiweissstoffe gespart würden. Die auch von Rosemann zitierte Stelle in der Arbeit von Rosenfeld lautet wörtlich folgendermassen: „Durch den Alkohol tritt eine Steigerung der Harnsäureausscheidung um $71 \mathrm{mg}=14 \%$ gegen die Vorperiode ein. Das lässt tief blicken; denn, da wir wissen, dass die Harnsäure von den nucleinhaltigen Eiweisskörpern abstammt, ist anzunehmen, dass in diesen ein $14 \%$ höherer Konsum stattgefunden hat. So entsteht

1) Herter and Smith, Observations on the Excretion of Uric Acid in Health and Desease. Newyork med. journ. vol. 55 p. 617.

2) H. L eber, Zur Physiologie und Pathologie der Harnsäureansscheidung beim Menschen. Berl. klin. Wochenschr. Bd. 34 S. 956. 1897.

3) A. Herrmann, Über die Abhängigkeit der Harnsäureausscheidung von Nahrungs- und Genussmitteln mit Rücksicht auf die Gicht. Deutsches Arch. f. klin. Medizin Bd. 43 S. 273. 1888.

4) G. Rosenfeld, Der Alkohol als Nahrungsmittel. Therapie der Gegenwart 1900 Heft 2.

5) H. Haeser, Der Einfluss des Alkohols auf die Harnsäureausscheidung. Inaug.-Diss. Greifswald 1901.

6) V. Tomaschny, Über den Verlauf der Harnsäureausscheidung beim Menschen. Inaug.-Diss. Greifswald 1898.

7) R. Rosemann, Über den Einfluss des Alkohols auf die Harnsäureausscheidung. Deutsche med. Wochenschr. 1901 Nr. 32. 
die Vorstellung, dass der Alkohol mit einer gewissen - sit venia verbo - Scheinheiligkeit die nucleinfreien Eiweisskörper geschont und gespart hat, und zwar in so hohem Masse, dass er unter den Nucleoalbuminen desto schlimmer wüten konnte, ohne dass es an der N-Bilanz sich verriet."

Roseman n glaubt aber auf Grund seiner Versuche, dass dieser Schluss zu weitgehend sei, und dass der Alkohol keinen Einfluss ausübe.

Auch die Versuche Beebe's ${ }^{1}$ ) geben verschiedene Resultate. 70-80 cem Alkohol, den Getränken beigemischt, bewirken eine Vermehrung der Harnsäuremengen, besonders wenn der Alkohol in Form von Portwein oder Bier gereicht wurde. In einem Fall, in dem nur $50 \mathrm{ccm}$ Alcohol absolutus mit Wasser verdünnt gegeben wurden, trat trotz Auftretens einer Diurese Verminderung der Harnsäureausscheidung auf. Da aus dem Referat, das uns zur Verfügung stand - die Arbeit ist in einer uns nicht zugänglichen Zeitschrift erschienen -, die genauen Versuchsbedingungen (Nahrung, Kotanalyse usw.) nicht ersichtlich sind, so kann diese Angabe nur mit Vorsicht verwertet werden. Jedoch darf wohl auf den Wirkungsunterschied grösserer und kleinerer Gaben auch an dieser Stelle hingewiesen werden. In der schon zitierten Arbeit von Pringsheim²) wird wieder darauf hingewiesen, dass trotz Eiweisssparung die Harnsäureausscheidung vermehrt sei. Pringsheim zieht wie Rosenfeld den Schluss, dass dies auf einem Zerfall von Nukleinen beruhe. Abgesehen von den schon besprochenen Einwänden, welche man gegen diese Ansicht geltend machen kann, muss auch der Umstand berücksichtigt werden, dass wir über das Entstehen der Harnsäure doch noch recht im Unklaren sind. Wenn wir eine Vermehrung der Harnsäure im Urin finden, so kann dies ja in der Tat daran liegen, dass mehr Harnsäure z. B. aus den Nukleinen gebildet worden ist; es könnte aber auch darauf zurückgeführt werden, dass die primär gebildete Harnsäure nicht im normalen Umfang zerstört wurde. Der Alkohol hätte alsdann auf Grund einer solchen Anschauung die Harnsäure zerstörende Tätigkeit gewisser Organe (Niere, Muskel,

1) S. P. Beebe, The Effect of Alcohol and Alcoholic Finids upon the Excretion of Uric Acid in Man. Report of the proceedings of the Am. physiolog. Society. Am. Journ. of Physiol. vol. 9 no. 5 p. 11.

2) Pringsheim, l. c. 
Leber) bzw. das „urikolytische“ Ferment [Schittenhelm ${ }^{1}$ )] hemmen können. Jedenfalls liegen die Verhältnisse viel zu kompliziert, als dass die Ansicht Pringsheim's mit Sicherheit zu Recht bestände.

In gewissem Zusammenhang mit der Harnsäureausscheidung steht auch die Phosphorsäureausscheidung. Auch darüber sind die vorhandenen Angaben wenig zufriedenstellend. Die einen [z. B. Strübing $\left.{ }^{2}\right)$ ] fanden bei Hunden eine Vermehrung der Phosphorsäure im Urin, Chittenden ${ }^{3}$ ) dagegen wieder geringe Verminderung der ausgeschiedenen Phosphorsäure. Beim Menschen wurde von Keller ${ }^{4}$ ), Bjerre ${ }^{5}$ ) und Leber ${ }^{6}$, auch Strübing ${ }^{7}$ ) eine Vermehrung der Phosphorsäureausscheidung gefunden. Doch halten diese Versuche aus verschiedenen Gründen einer strengen Kritik nicht stand (Rosenfeld). Auch ist gegen dièse Versuche der Einwand zu erbeben, dass die Dosierung vernachlässigt worden ist. So sagt z. B. Strübing, dass eine seiner Versuchspersonen Alkohol bis zur "Ebrietas" bekam. Diese Ausserachtlassung der Mengen des wirksamen Agens spielt nach unserer Ansicht eine geradezu verhängnisvolle Rolle. Es ist merkwürdig, dass dieser Umstand bisher so sehr vernachlässigt wurde, der doch bei allen anderen pharmakologiseh wirksamen Substanzen so seharf betont wird. Es sei da nur an ein ganz landläufiges Beispiel erinnert, an die Wirkung der Digitalis, die sich bei verschiedener Dosierung bezüglich ihrer Beziehung zum Vagus recht verschieden verhält.

\section{Eigene Versuche.}

Als Versuchstiere wählten wir Kaninchen. Die Gründe, welche uns bestimmten, Kaninchen den Hunden vorzuziehen, war vor allem

1) A. Schittenhelm, Über die Harnsäurebildung und die Harnsäurezersetzung in den Auszügen der Rinderorgane. Zeitschr. f. physiol. Chemie Bd. 45 S. 121.

2) P. Strübing, Über die. Phosphorsäure im Urin unter dem Finflusse excitierender und deprimierender Mittel. Arch. f. exper. Path. u. Pharm. Bd. 6 S. 266. 1877.

3) Chittenden, 1. c.

4). Keller (zit. nach Rosenfeld). Zeitschr. f. physiol. Chemie Bd. 13.

5) Bjerre, l. c.

6) Leber, l. c.

7) Strrübing, l. c. 
der Umstand, dass Hunde in engen Käfigen krank werden, zum mindesten eine ziemlich erhebliche Eiweissausscheidung im Urin auftritt, und dass Hunde von gleicher Grösse, gleichem Alter und gleicher Rasse in genügender Zahl schwer oder gar nicht zu beschaffen sind. Auch mussten wir uns sagen, dass an Hunden nur einzelne Versuche möglich waren, während an Kaninchen grössere Versuchsreihen in oftmaliger Wiederholung angestellt werden konnten. Dann durfte anch bei Kaninchen bei vollkommener Nahrungsentziehung und geringerer oder grösserer Flüssigkeitszufuhr erfahrungsgemäss auf eine kürzere Lebensdauer gerechnet werden als bei Hunden, was bei Fehlschlagen einer Versuchsserie infolge falscher Dosierung des Alkohols oder anderer nicht vorauszusehender Umstände eine Wiederholung der Versuche schwieriger gestaltet hätte. Trotzdessen waren wir uns bewusst, dass Kaninchen für Stoffwechselversuche etwas weniger geeignet sind als Hunde; doch erschienen die ersten Gründe massgebender und stichhaltiger zu sein als die Bedenken, so dass wir unsere Versuche schliesslich nur an Kaninchen anstellten.

\section{Versuchsreihe.}

Die erste Versuchsserie erstreckte sich auf sechs Tiere, welche in Zinkkäfigen untergebracht wurden, die ihnen einige Bewegungsfreiheit gewährten und das gesonderte Auffangen des Urins gestatteten. Das Körpergewicht wurde täglich festgestellt, der Urin gemessen, sein spezifisches Gewicht, seine Reaktion untersucht und auf Anwesenheit von Eiweiss und Zucker geprüft. Stoffwechseluntersuchungen wurden bei dieser Serie noch nicht gemacht, da sie lediglich den Zweck verfolgte, die Einwirkung des Alkohols auf die Lebensdauer zu konstatieren. Bevor den Tieren die Nahrung entzogen wurde, wurden sie 6 Tage lang bei gleichbleibender Nahrung belassen, die aus $150 \mathrm{~g}$ Mohrrüben und $50 \mathrm{~g}$ Hafer bestand. Am Nachmittag des sechsten Tages erhielten vier der Tiere 120 cem 10\% igen (Volumprozent) Alkohols mittels Schlundsonde in den Magen, die übrigen zwei Tiere dienten als Kontrolltiere und bekamen $120 \mathrm{ccm}$ Wasser einverleibt. Die Einzelheiten ergeben sich aus den nachstehenden Tabellen. 


\section{Serie.}

Tabelle I. Kaninchen 1.

\begin{tabular}{|c|c|c|c|c|c|c|c|c|}
\hline $\begin{array}{c}\text { Datum } \\
1904\end{array}$ & हैं & 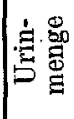 & 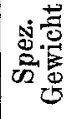 & $\begin{array}{c}\text { Re- } \\
\text { aktion }\end{array}$ & $\begin{array}{c}\text { Al- } \\
\text { bumen }\end{array}$ & 递 & $\begin{array}{c}\text { Futter bzw. } \\
\text { Alkohol }\end{array}$ & Bemerkungen \\
\hline 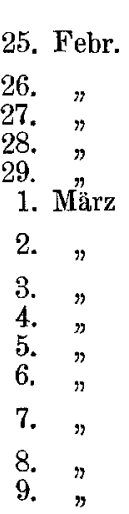 & $\begin{array}{l}1740 \\
1680 \\
1620 \\
- \\
1570 \\
1575 \\
1460\end{array}$ & $\begin{array}{r}\text { cem } \\
- \\
58 \\
80 \\
136 \\
138 \\
190\end{array}$ & $\begin{array}{c}1018 \\
1017 \\
1019 \\
- \\
1011 \\
1012 \\
1010\end{array}$ & $\begin{array}{c}\text { - } \\
\text { alkal. } \\
n \\
" \\
" \\
" \\
" \\
\text { sohwach alk. } \\
" \\
" \\
" \\
\text { sauer }\end{array}$ & $\begin{array}{c}- \\
- \\
- \\
- \\
- \\
\text { schwache } \\
\text { Truibung } \\
+ \\
+ \\
- \\
- \\
\text { soliwache } \\
\text { Tribung }\end{array}$ & - & $\begin{array}{c}150 \mathrm{~g} \text { Mohrrüben, } \\
50 \mathrm{~g} \text { Hafer } \\
" \\
" \\
120 \mathrm{ccm} \text { "Wasser } \\
"\end{array}$ & $\begin{array}{l}\text { Reaktion } \\
\text { Eiwoiss : Kochprobe } \\
\text { Zucker : Trommer } \\
\text { Alkalinitat: Iackmus }\end{array}$ \\
\hline
\end{tabular}

Tabelle II. Kaninchen 2.

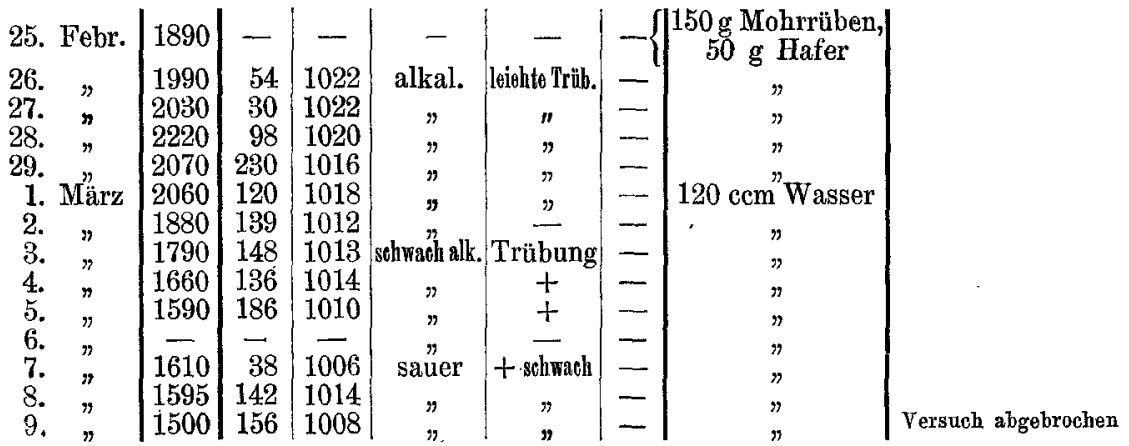

Tabelle III. Kaninchen 3.

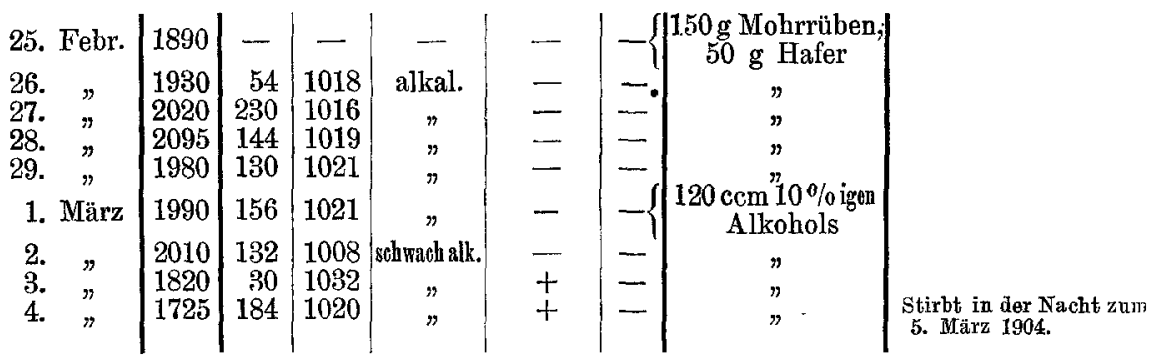

Sektion ergab: Herz und Lunge ohne Besonderheiten. Nieren: feste Konsistenz, hellbraune Färbung, anscheinend geringer Blutgehalt. Rinde und 
Mark durch eine rotbraune Zwischenzone deutlich geschieden. Färbung des Markes mit einem Stich ins Gelbliche. Leber: ziemlich feste Konsistenz, undeutliche Zeichnung, hellbraune Färbung. Magen: besonders auf der rötlich verfärbten Mukosa der grossen Kurvatur eine grössere Anzahl Flecken von schwarzbrauner bis schwarzer Färbung, welche von verschiedenem Durchmesser scharf, gegen die Umgebung abgesetzt, über die Oberfläche erhaben sind.

Diagnose: Beginnende Nephritis, Hämorrhagien in der Magenschleimhaut.

Tabelle IV. Kaninchen 4 .

\begin{tabular}{|c|c|c|c|c|c|c|c|c|}
\hline $\begin{array}{c}\text { Datum } \\
1904\end{array}$ & 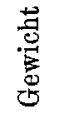 & 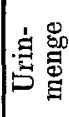 & 递 & $\begin{array}{l}\text { Re- } \\
\text { aktion }\end{array}$ & $\begin{array}{c}\text { Al- } \\
\text { bumen }\end{array}$ & 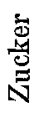 & $\begin{array}{c}\text { Futter bzw. } \\
\text { Alkohol }\end{array}$ & Bemerkungen \\
\hline 25. Febr. & $\stackrel{g}{g}$ & ccm & 一 & - & - & -\{ & $\begin{array}{c}150 \mathrm{~g} \text { Mohrrüben, } \\
50 \mathrm{~g} \text { Hafer }\end{array}$ & \\
\hline 26. & 1890 & 81 & 1020 & alkal. & - & - & 0 & \\
\hline 27. " & 1770 & 30 & 1023 & $"$ & - & - & $"$ & \\
\hline $38 . \quad 7$ & 1687 & 46 & 1036 & $n$ & - & - & $"$ & \\
\hline 13. & 1670 & 90 & 1040 & $"$ & Trïhnng & 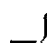 & $120 \mathrm{ccm}^{\prime \prime} 10 \%$ igon & \\
\hline 1. Marz & 1690 & 04 & 1045 & $m$ & 2tuing & ) & Alkohols & \\
\hline $\begin{array}{l}2 . \quad " \\
3 .\end{array}$ & 1090 & 38 & - & $"$ & $\stackrel{+}{-}$ & - & $"$ & $\begin{array}{l}\text { Stirbt in der Nacht rom } \\
\text { 2. zum 3. März 1904, }\end{array}$ \\
\hline
\end{tabular}

Sektion ergab: Keine Besonderheiten. Lungen überall lufthaltig.

Tabelle V. Kaninchen 5 .

\begin{tabular}{|c|c|c|c|c|c|c|c|c|}
\hline Febr. Fe. & 1870 & - & - & - & 一 & & $150 \mathrm{~g}$ Mohrrüben, & \\
\hline & 1990 & 100 & 1016 & alkal. & $\ldots$ & -1 & 50 g Hafer & \\
\hline$"$ & 1940 & 210 & 1016 & arkate & - & - & $"$ & \\
\hline$n$ & 2010 & 170 & 1017 & $"$ & laichte Trüb. & - & $"$ & \\
\hline 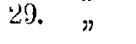 & 1900 & 148 & 1014 & $"$ & is & - & 100 "1010 & \\
\hline 1. März & 1930 & 118 & 1014 & " & + & 一 & $\begin{array}{c}120 \mathrm{ccm} 10 \% \text { igen } \\
\text { Alkohols }\end{array}$ & \\
\hline " & 1800 & 200 & 1009 & & leich te Trüb. & 一 & $n$ & \\
\hline$"$ & $\begin{array}{l}1740 \\
1510\end{array}$ & $\begin{array}{l}188 \\
146\end{array}$ & $\begin{array}{l}1014 \\
1014\end{array}$ & schwach alk. & $\stackrel{t}{t}$ & & 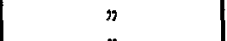 & \\
\hline$"$ & 1430 & 148 & 1019 & alkal. & + & - & $"$ & $\begin{array}{l}\text { 6. März } 1904 \text { gestorben, } \\
\text { nachdem es seit demi } \\
\text { 5. März } i \text {, d.Agoneliegt. }\end{array}$ \\
\hline
\end{tabular}

Sekti o n ergab: Fettpolster gering. Schleimhaut des Larynx und der Trachea gerötet. Lungen lufthaltig, an einzelnen Stellen rote nicht abwischbare Flecken. Herz ohne Besonderheiten. Nieren: Konsistenz fest. Starker Blutreichtum. Mark gelblich. Leber: undeutliche Zeichnung, starke Blutfüllung. Rotbraune Färbung. In der rosenrot verfärbten Magenschleimhaut der grossen Kurvatur und des Pylorus schwarzbraune, über die Oberfläche erhabene Flecken, zum Teil strichförmig angeordnet. Auch Duodenalschleimhaut leicht gerötet.

Diagnose: Hämorrhagien in der Magenschleimhant. Beginnende Nephritis. 
Tabelle VI. Kaninchen 6 .

\begin{tabular}{|c|c|c|c|c|c|c|c|c|}
\hline $\begin{array}{c}\text { Datum } \\
1904\end{array}$ & & 息 & 悹苞 & $\begin{array}{c}\text { Re- } \\
\text { aktion }\end{array}$ & $\begin{array}{c}\text { Al- } \\
\text { bumen }\end{array}$ & 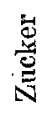 & $\begin{array}{c}\text { Futter bzw. } \\
\text { Alkohol }\end{array}$ & Bemerkungen \\
\hline 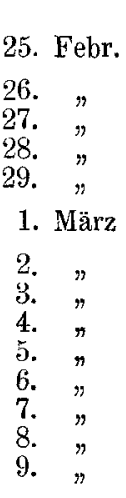 & $\begin{array}{r}1910 \\
1780 \\
1690 \\
1610 \\
- \\
1520 \\
1550 \\
1420\end{array}$ & $\begin{array}{r}262 \\
176 \\
48 \\
78 \\
-178 \\
44 \\
124\end{array}$ & $\begin{array}{l}1007 \\
1007 \\
1013 \\
1022 \\
-\overline{1015} \\
1015 \\
1010\end{array}$ & $\begin{array}{c}- \\
\text { alkal. } \\
" \\
" \\
" \\
" \\
\text { selwwhth alk. } \\
\text { sauer } \\
\text { sauer } \\
" \\
"\end{array}$ & $\begin{array}{c}- \\
- \\
+ \\
+ \\
+ \\
+ \\
+ \\
+ \\
+ \\
\text { stark } \\
+ \\
+\end{array}$ & $\begin{array}{l}- \\
- \\
- \\
+? \\
+? \\
-\{ \\
- \\
+ \\
- \\
- \\
-\end{array}$ & $\begin{array}{c}150 \mathrm{~g} \text { Mohrrübell, } \\
50 \mathrm{~g} \text { Hafer } \\
" \\
" \\
" \\
120 \mathrm{ccm} " 10 \% \text { igen } \\
\text { Alkohols } \\
" \\
" \\
" \\
" \\
" \\
"\end{array}$ & In der Nacht gestorben \\
\hline
\end{tabular}

Sektio n erga b: Geringes Fettpolster. Herz: ohne Besonderheiten. Lungen: eine alte Narbe, sonst lufthaltig. Nieren: Mark und Rinde durch eine braunrote Zwischenschicht scharf geschieden, Rinde mit gelblichem Timbre. Leber: an einigen Stellen gelblich verfärbt, sonst ohne Besonderheiten. Magen: in der Schleimhaut des Fundus zahlreiche Hämorrhagien, wie sie auch bei den anderen Kaninchen schon oben beschrieben worden sind. Duodenalschleimhaut: gerötet.

Diagnose: Nephritis acuta. Hämorrhagien im Magen.

Es zeigt sich also, dass der Alkohol von 10 Volumprozent in der verabreichten Gabe von $120 \mathrm{ccm}$ die Lebensdauer der Tiere erheblich verkürzt. Die Hungertiere, welchen nur $120 \mathrm{ccm}$ Wasser verabreicht wurden, lebten noch, als auch das letzte mit Alkohol behandelte Tier zugrunde gegangen war, und erholten sich wieder vollkommen, als ihnen die frühere Nahrung gegeben wurde. Die Alkoholtiere starben bei ungefähr gleichem Anfangsgewicht von 1990, 1710,1930 und $1970 \mathrm{~g}$ nach $4,3,5$ und 9 Tagen. Bei diesen Tieren zeigten sich auch ziemlich erhebliche annatomische Läsionen, welche besonders in Blutungen der Magensehleimhaut und beginnender Entzündung des Nierenparenchyms bestanden, Läsionen, welche schon an und für sich wohl den Tod hätten bedingen können.

\section{Versuchsreihe.}

In der zweiten Versuchsserie glaubten wir die Schädigungen von seiten des Alkohols dadurch besser vermeiden zu können, dass 
wir den Tieren weniger Alkohol darreichten. Zwei derselben er-

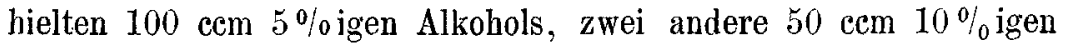
Alkohols in physiologischer Kochsalzlösung. Zwei weitere Tiere dienten als Kontrolltiere, und das eine von diesen bekam $50 \mathrm{ccm}$ physiologischer Kochsalzlösung, das andere $100 \mathrm{ccm}$. Bevor die Nahrung von täglich $150 \mathrm{~g}$ Mohrrüben und $50 \mathrm{~g}$ Hafer ausgesetzt wurde, wurden die Tiere 5 Tage lang voll ernährt und beobachtet. Die Einzelheiten sind aus den nachstehenden Tabellen zu ersehen.

Serie II.

Tabelle VII. Kaninchen 7 .

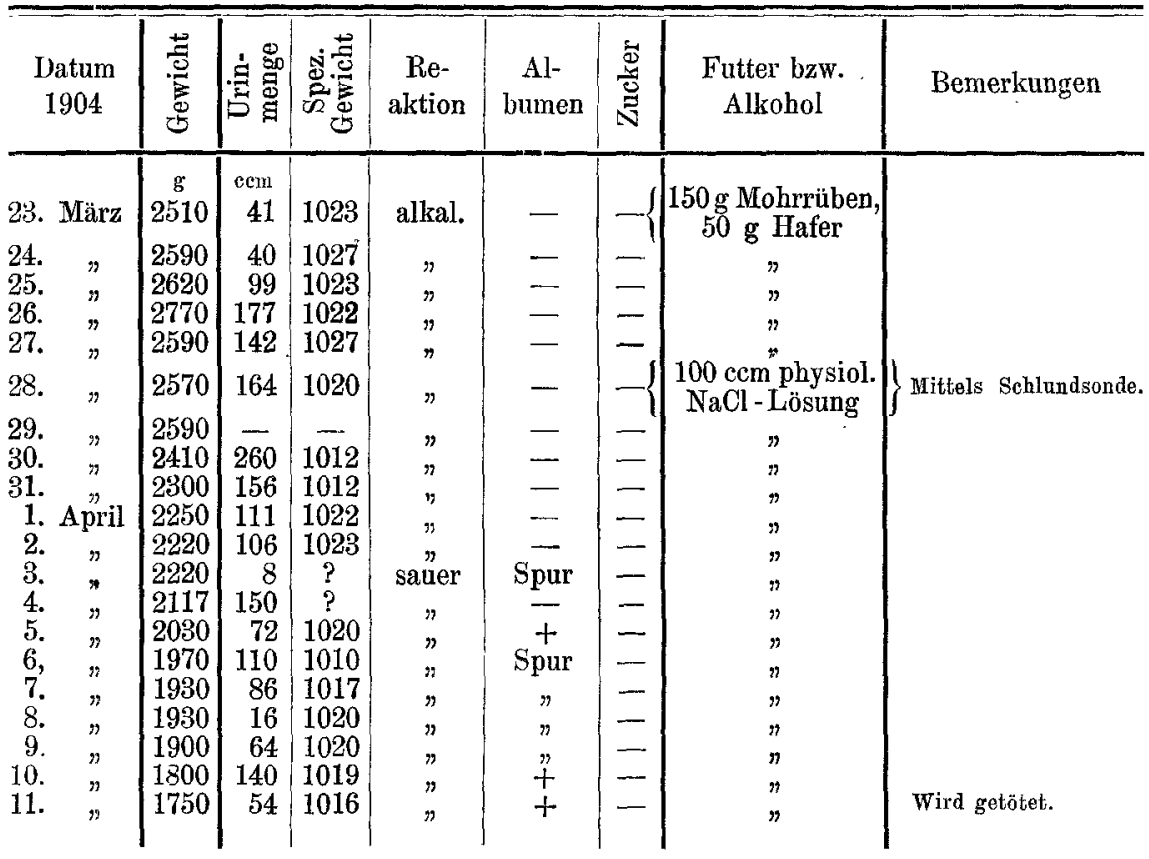

Sektion ergab: Keinerlei pathologisch-anatomische Veränderungen.

Tabelle VIII. Kaninchen 8.

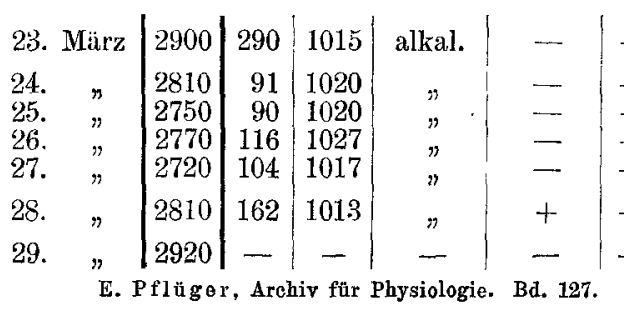

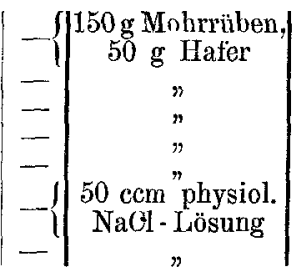


Tabelle VIII. (Fortsetzung.)

\begin{tabular}{|c|c|c|c|c|c|c|c|c|}
\hline $\begin{array}{c}\text { Datum } \\
1904\end{array}$ & 苞 & 常离 & 总蓄 & $\begin{array}{c}\text { Re- } \\
\text { aktion }\end{array}$ & $\begin{array}{c}\text { Al- } \\
\text { bumen }\end{array}$ & 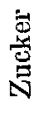 & $\begin{array}{c}\text { Futter bzw. } \\
\text { Alkohol }\end{array}$ & Bemerkungen \\
\hline 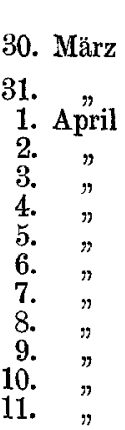 & $\begin{array}{l}\mathrm{g} \\
2870 \\
2670 \\
2610 \\
2510 \\
2360 \\
2365 \\
2220 \\
2220 \\
2115 \\
2090 \\
2000 \\
1930 \\
1910\end{array}$ & $\begin{array}{l}\mathrm{ecm} \\
- \\
170 \\
42 \\
2 \\
- \\
- \\
168 \\
10 \\
150 \\
62 \\
30 \\
114 \\
-\end{array}$ & $\begin{array}{c}\overline{ } \\
1015 \\
1035 \\
\overline{-} \\
\overline{1015} \\
\overline{1020} \\
1020 \\
1022 \\
1022 \\
1025\end{array}$ & $\begin{array}{c}- \\
\text { alkal. } \\
" \\
\overline{-} \\
\text { sauer } \\
- \\
\text { sauer } \\
" \\
" \\
" \\
"\end{array}$ & $\begin{array}{l}- \\
\frac{+}{+} \\
\overline{-} \\
\text { Spur } \\
\text { Spur } \\
" \\
" \\
"\end{array}$ & $\begin{array}{l}- \\
- \\
- \\
- \\
- \\
- \\
- \\
-\end{array}$ & $\begin{array}{c}50 \mathrm{ccm} \text { physiol. } \\
\text { NaCl-Lösung } \\
" \\
" \\
" \\
" \\
" \\
" \\
" \\
" \\
" \\
"\end{array}$ & Lebt. Vers abgebrochen \\
\hline
\end{tabular}

Bei gewöhnlicher Nahrung erholt sich das Tier vollkommen.

\section{Tabelle IX. Kaninchen 9 .}

\begin{tabular}{|c|c|c|c|c|c|c|c|c|}
\hline 23. März & 2600 & 120 & 1017 & alkal. & - & -\{ & $\begin{array}{c}150 \mathrm{~g} \text { Mohrrüben. } \\
50 \mathrm{~g} \text { Hafer }\end{array}$ & \\
\hline 24. & 2520 & 142 & 1020 & 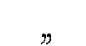 & Spur & - & ou g nater & \\
\hline 25. & 2500 & 140 & 1023 & " & - & - & $"$ & \\
\hline 26. & 2580 & 92 & 1027 & $"$ & - & - & $"$ & \\
\hline 27. & & 74 & 1022 & $n$ & - & - & $100 \mathrm{ccm}^{\prime \prime} 5 \%$ igen & \\
\hline 28. & 2610 & 189 & 1023 & $n$ & - & -\{ & Alkohols & \\
\hline 29. & 2700 & - & - & " & - & - & $n$ & \\
\hline $30 . \quad "$ & 2550 & 264 & 1013 & 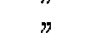 & - & - & $"$ & \\
\hline 31. April & $\begin{array}{l}2410 \\
2330\end{array}$ & $\begin{array}{l}76 \\
97\end{array}$ & $\begin{array}{l}1024 \\
1018\end{array}$ & $n$ & - & - & $"$ & \\
\hline 2. $"$ & 2270 & 132 & 1025 & sauer & + & - & $"$ & $\begin{array}{l}\text { Chernisch u. mikroskop. } \\
\text { Blut im Urin. }\end{array}$ \\
\hline $\begin{array}{l}3 . \quad \\
4 .\end{array}$ & $\begin{array}{l}2090 \\
1940\end{array}$ & $\begin{array}{l}158 \\
120\end{array}$ & $\begin{array}{l}1020 \\
1009\end{array}$ & $"$ & + & - & $"$ & 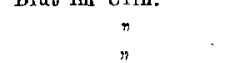 \\
\hline 5. & 1850 & 100 & 1035 & $"$ & + & - & $"$ & Kein Blut." \\
\hline 6. & 1795 & 104 & 1035 & $n$ & $t$ & - & $"$ & \\
\hline$"$ & 1785 & 26 & 1035 & 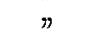 & + & 一 & $"$ & \\
\hline$"$ & $\begin{array}{l}1760 \\
1610\end{array}$ & $\begin{array}{r}60 \\
198\end{array}$ & 1035 & $"$ & + & - & $"$ & \\
\hline 10. & 1470 & 38 & 1035 & $"$ & + & - & $"$ & Liegt im Sterben. \\
\hline
\end{tabular}

Sektion ergab: Nephritis acuta. Hämorrhagien in der Magenschleimhaut.

\section{Tabelle X. Kaninchen 10 .}

\begin{tabular}{|c|c|c|c|c|c|c|c|}
\hline 23. März & 2610 & 159 & 1016 & alkal. & - & -\{ & $150 \mathrm{~g}$ Mohrrüben, \\
\hline 24. & 2570 & 104 & 1019 & & 一 & - & $"$ \\
\hline$"$ & 2480 & 178 & 1020 & $n$ & 一 & - & $"$ \\
\hline " & 2520 & 70 & 1023 & $"$ & - & - & $\%$ \\
\hline & 12500 & - & - & - & - & & $"$ \\
\hline
\end{tabular}


Tabelle X. (Fortsetzung.)

\begin{tabular}{|c|c|c|c|c|c|c|c|c|}
\hline $\begin{array}{c}\text { Datum } \\
1904\end{array}$ & 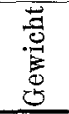 &. & 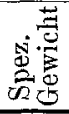 & $\begin{array}{c}\text { Re- } \\
\text { aktion }\end{array}$ & $\begin{array}{c}\text { Al- } \\
\text { bumen }\end{array}$ & 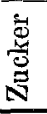 & $\begin{array}{c}\text { Futter bzw. } \\
\text { Alkohol }\end{array}$ & Bemerkungen \\
\hline 28. März & $\begin{array}{c}g \\
2430\end{array}$ & $\begin{array}{l}\text { cem } \\
148\end{array}$ & - & alkal. & - & & $100 \mathrm{ccm} 5 \%$ igen & \\
\hline & 2510 & - & 1018 & 一 & - & - & Alkonols & \\
\hline & 2420 & 244 & 1013 & alkal. & + & - & $"$ & \\
\hline $31 . "$ & 2290 & 96 & 1019 & 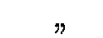 & + & - & $"$ & \\
\hline 1. April & 2110 & 80 & 1017 & sauer & + & - & $"$ & \\
\hline 2. & 2070 & 166 & 1027 & 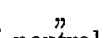 & + & - & $"$ & \\
\hline 3. & 2040 & 68 & 1020 & neutral & + & - & $"$ & \\
\hline 4. & 1900 & $\overline{160}$ & $1 \overline{017}$ & - & - & - & $"$ & \\
\hline 5. & 1970 & 160 & 1017 & sauer & + & - & $"$ & \\
\hline 7 & 1615 & 110 & 1040 & $"$ & + & - & $n$ & \\
\hline 8. & 1010 & 110 & 1019 & $"$ & $t$ & 一 & $"$ & \\
\hline 9. & 1510 & 20 & $100 x$ & $"$ & + & - & $"$ & Stirbt in der Nacht. \\
\hline
\end{tabular}

Tabelle XI. Kaninchen 11.

\begin{tabular}{|c|c|c|c|c|c|c|c|c|}
\hline 23. März & 2650 & 175 & 1019 & alkal & - & & $150 \mathrm{~g}$ Mohrrüben, & \\
\hline 24. & 0575 & & 20 & & & 1 & $50 \mathrm{~g}$ Hafer & \\
\hline 25. & $\begin{array}{l}2575 \\
2560\end{array}$ & 138 & 1023 & $"$ & - & - & $"$ & \\
\hline$"$ & 2540 & $\begin{array}{l}108 \\
108\end{array}$ & $\begin{array}{l}1020 \\
1031\end{array}$ & $"$ & - & - & $m$ & \\
\hline$"$ & 2510 & - & - & - & - & - & $"$ & \\
\hline 28. & 2500 & 158 & 1019 & alkal. & - & -\{ & $\begin{array}{c}50 \mathrm{ccm} 10 \% \text { igen } \\
\text { Alkohols }\end{array}$ & Durchfall, \\
\hline " & 2550 & - & - & - & - & - & MIAULUID & Kein Durchfall mehr. \\
\hline$"$ & 2340 & 195 & 1019 & alkal. & - & $\ldots$ & " & \\
\hline 31. $"$ & 2190 & 150 & 1019 & $n$ & 一 & - & $n$ & \\
\hline 1. April & 2180 & $\overline{10}$ & $\overline{1000}$ & $n$ & - & - & $"$ & \\
\hline$"$ & $\begin{array}{l}2080 \\
2050\end{array}$ & 164 & 1029 & $"$ & + & - & " & \\
\hline$"$ & 2110 & $\overline{176}$ & 1024 & sauer & $\bar{t}$ & - & $"$ & \\
\hline 5. & 2010 & - & - & Deter & - & - & $"$ & \\
\hline ", & 1800 & 122 & 1085 & $"$ & + & - & $"$ & \\
\hline$n$ & 1785 & 136 & 1019 & $n$ & + & - & " & \\
\hline$n$ & 1750 & $\overline{c o}$ & $\overline{10}$ & $n$ & - & - & $"$ & \\
\hline$\eta$ & $\begin{array}{l}1>0 \\
1700\end{array}$ & $\begin{array}{r}60 \\
128\end{array}$ & $\begin{array}{l}1018 \\
1023\end{array}$ & $"$ & + & - & " & \\
\hline & 1490 & 100 & 1023 & $"$ & + & - & $n$ & Im Urin Blut. Stirbt in \\
\hline
\end{tabular}
Sektion ergab: Nephritis hämorrhagica. Keine Hämorrhagien im Magen.

Tabelle XII. Kaninchen 12.

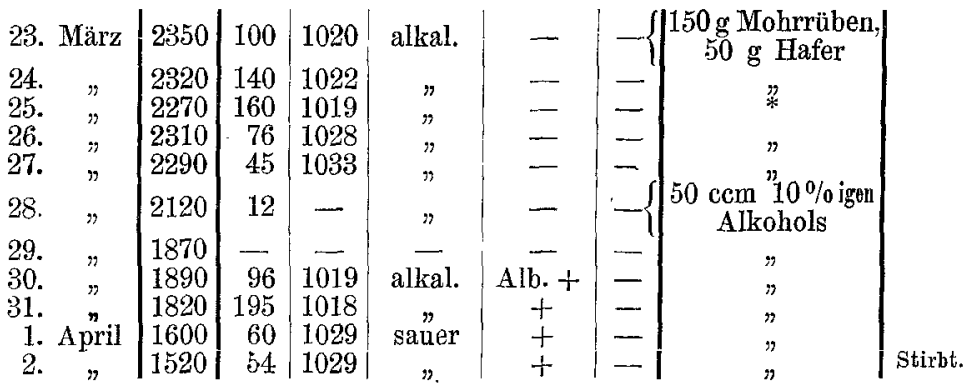

Sektion ergab: Nephritis acuta. Hämorrhagien in der Magenschleimhaut. 
Auch aus dieser Versuchsreihe ergibt sich die Tatsache, dass der Alkohol in der verabreichten Gabe bei sonstiger Nahrungsentziehung den Tod der Tiere beschleunigt. Die schweren anatomischen Veränderungen, Blutungen in der Magenschleimhant, sowie vor allen Dingen die degenerativen Vorgänge in der Niere, zeigen mit Sicherheit, dass der Alkohol auch bei dieser Versuchsreihe stark toxisch gewirkt hat. Nur ganz im Anfang der Alkoholdarreichung könnte es den Ansehein haben, als ob der Alkohol den Abfall des Körpergewichtes wenigstens bei den Tieren Nr. 9, 10 und 11 etwas hingehalten hätte. So nimmt Kaninchen Nr. 7 während der ersten 3 Tage der Inanition um 7,8\% seines Anfangsgewichtes ab, Nr. 8 um 12,6\%, Nr. 9, 10 und 11 jedoch nur um $0,4 \%$, $3,2 \%$ und $6 \%$. Man muss zugestehen, dass die Unterschiede nur gering sind, aber sie legten uns doch den Gedanken nahe, dass es vielleicht möglich sei, das Leben der Versuchstiere zu verlängern, wenn die richtige Dosierung des Alkohols getroffen würde, welche die toxische Wirkung vermied. Und so begannen wir denn eine neue Serie, allerdings unter gänzlich anderen Versuchsbedingungen.

\section{Versuchsreihe.}

Auch zu dieser Serie wurden sechs Tiere eingestellt. Je zwei, ein grösseres und ein kleineres, wurden in einen Glaskäfig gebracht. Die Käfige waren grosse Säureballons aus grünem Glase, wie sie für den Versand und die Aufbewahrung von Schwefelsäure gebraucht werden. Die Böden wurden in der bekannten Weise abgeschnitten, die Ballons umgestülpt, so dass der Urin der in ihnen untergebrachten Tiere durch den Hals des Ballons in ein untergestelltes Gefäss ablaufen konnte, in welchem sich $3 \mathrm{ccm}$ Toluol befanden. Der in den Käfigen noch anhaftende Urin wurde mit destilliertem Wasser abgespült und gemessen. Die Tiere sassen auf einem starken Drahtgitter, dessen Maschenweite so gross gewählt war, dass der Kot durehfallen konnte. Durch einen Glastrichter, welcher auf das Urinsammelgefäss aufgesetzt war, und in welchem sich ein zweiter eingepasster Trichter aus engem Drahtgeflecht befand, konnte es vermieden werden, dass der Kot in den Urin fiel und ausgelaugt wurde. Alle 2 Tage wurde das Körpergewicht der Tiere festgestellt, die Urinmenge von je zwei in einem Käfig befindlichen Kaninehen. gemessen, nachdem die Blase durch vorsichtiges Ausdrücken entleert war. In einem aliquoten Teil des Urins bestimmten wir dann den 
Stickstoff nach Kjeldahl, den Gesamtschwefel nach H. Schulz ${ }^{1}$ ) und in späteren Serien auch die Phosphorsäure durch Titration mit Uranoxyd. Die Stickstoffbestimmung nach $\mathrm{Kjeldahl}$ wurde in der gewöhnlichen Weise vorgenommen: Zerstörung mit konzentrierter Schwefelsäure unter Zusatz von Kupfersulfat und Kalium sulfuricum, Neutralisation durch 25\% ige Natronlauge und Destillation unter Zusatz von Talkum in $\mathrm{n} / 10$ Schwefelsäure, Titration durch n/10 Natronlauge mit Cochenilletinktur als Indikator. Die Schwefelbestimmung nach H. Schulz, auf deren schnelle, bequeme und genaue Ausführung hingewiesen zu werden verdient, wurde zunächst in der III. Serie so vorgenommen, dass mittels rauchender Salpetersäure 5-10 ccm Urin in einem Glaskölbchen oxydiert wurden, welches seitwärts einen Glasrohransatz trug, der in ein mit Wasser gefülltes Becherglas tauchte. Nach vollkommener Zerstörung des Urins wurde. der Salzrückstand in Wasser gelöst, mit Salzsäure gekocht, die Schwefelsäure mit Baryumchlorid gefällt und als schwefelsaurer Baryt gewogen. In den späteren Versuchsreihen bedienten wir uns der neuen Modifikationen von $\mathrm{H} . \mathrm{Schul}^{2}$ ). Der Urin wird dabei in einem kleinen Kjeldahlkolben mit rauchender Salpetersäure oxydiert, indem die Flüssigkeit bis zur Trockenheit eingedampft wird, wobei sich dichte gelbbraune Wolken entwickeln; der Rückstand wird in Wasser gelöst, mit Salzsäure aufgekocht und die Salzlösung in ein Beqherglas übergespült, in welchem dann die schwefelsauren Salze als Baryumsulfat gefällt wurden. Die Niederschläge wurden dann abfiltriert und gewogen. Diese einfache Methode ermöglichte es uns, an einem Tage zwölf Schwefelbestimmungen auszuführen, was mit den anderen Methoden nach Mohr, bei der Einschmelzmethode mit Salpeter oder nach Carius nicht möglich gewesen wäre. Die Zahlen, welche nach dieser neuen Methode gewonnen werden, stimmen mit den nach der Carius'schen Methode erhaltenen gut überein.

Bei der Bestimmung der Pbosphorsäure hielten wir uns genau an die Vorschriften, wie sie Huppert ${ }^{3}$ ) in seinem Lehrbuch wieder-

1) H. Schulz, Die Bestimmung des gesamten Schwefelgehaltes im Harne. Arch. f. d. ges. Physiol. Bd. 57 s. 57. 1894.

2) H. Scth ulz, Die quantitative Bestimmung des Gesamtschwefels im Harn. Arch. f. d. ges. Physiol. Bd. 121 S. 114. 1907.

3) H. Huppert, Neubauer und Vogel, Analyse des Harns. Wiesbaden 1908 . 
gibt. Es wurden bei allen Analysen immer je zwei Kontrollbestimmungen vorgenommen. Nur vom Kot, dessen Stickstoffgehalt wir feststellten, wurde eine einzige Analyse gemacht, da wir auf die Kotanalysen bei Kaninchen wenig Wert legen. Es ist bekannt, dass die Kaninchen die Ingesta ausserordentlich lange in dem immer

Serie III.

Tabelle XIII.

\begin{tabular}{|c|c|c|c|c|c|c|c|}
\hline \multirow{2}{*}{$\begin{array}{c}\text { Datum } \\
1907\end{array}$} & \multicolumn{2}{|c|}{ Körpergew. } & \multicolumn{2}{|c|}{ Gewichtsverlust in $g$ und $\%$} & \multirow{2}{*}{$\begin{array}{l}\text { Urin- } \\
\text { menge }\end{array}$} & \multicolumn{2}{|c|}{ N-Bestimmung } \\
\hline & a & $\mathrm{b}$ & a & $\mathrm{b}$ & & inl ganzen & auf $1 \mathrm{~kg}$ Giew. \\
\hline 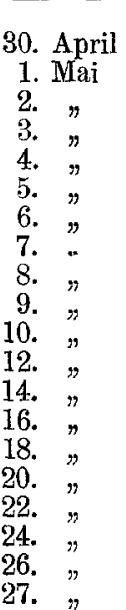 & $\begin{array}{c}\mathrm{g} \\
1787 \\
1773 \\
1753 \\
1745 \\
1743 \\
1679 \\
1720 \\
1770 \\
1771 \\
1738 \\
1745 \\
1550 \\
1415 \\
1378 \\
1270 \\
1118\end{array}$ & $\begin{array}{l}g \\
2180 \\
2168 \\
2170 \\
2140 \\
2135 \\
2118 \\
2153 \\
2155 \\
2202 \\
2088 \\
2025 \\
1925 \\
1812 \\
1712 \\
1637 \\
1560 \\
1483 \\
1390 \\
1255 \\
1235\end{array}$ & $\begin{array}{r}195 \mathrm{~g}=11,2 \% \\
135 "=7,7 \% \\
37 "=2,1 " \\
108 \%=6,2 " \\
152 "=8,7 "\end{array}$ & $\begin{aligned} 100 \mathrm{~g} & =4,9 \% \\
113 & =5,6 \% \\
100 " & =4,9 " \\
77 & =3,8 " \\
77 & =3,8 \% \\
77 & =3,8 \% \\
93 & =3,8 \% \\
135 " & =4,6 " \\
20 & =6,7 "\end{aligned}$ & $\begin{array}{l}719 \\
630 \\
600 \\
830 \\
790 \\
790 \\
780 \\
645 \\
660 \\
610 \\
750 \\
250 \\
140 \\
115 \\
155 \\
150 \\
50 \\
50 \\
80 \\
25\end{array}$ & $\begin{array}{l}1,3500 \\
1,4342 \\
2,1684 \\
2,9040 \\
2,5139 \\
2,9700 \\
2,5625 \\
1,5210 \\
1,7360 \\
2,9040 \\
0,41150\end{array}$ & $\begin{array}{l}0,35 \\
0,38 \\
0,62 \\
0,90 \\
0,81 \\
1,00 \\
1,20 \\
1,02 \\
1,25 \\
2,30 \\
0,67\end{array}$ \\
\hline
\end{tabular}

Tabelle XIV.

\begin{tabular}{|c|c|c|c|c|c|}
\hline \multirow{2}{*}{$\begin{array}{c}\text { Datum } \\
1907\end{array}$} & \multicolumn{2}{|c|}{ Körpergewicht } & \multicolumn{2}{|c|}{ Gewichtsverlust in $\mathrm{g}$ und $\%$} & \multirow{2}{*}{$\begin{array}{l}\text { Urin- } \\
\text { menge }\end{array}$} \\
\hline & $a$ & $b$ & $a$ & $b$ & \\
\hline $\begin{aligned} & \text { 30. April } \\
& 6 . \\
& 7 . \text { Mai } \\
& 8 . " \\
& 9 . " \\
& 10 . " \\
& 12 . " \\
& 14 . " \\
& 16 . " \\
& 18 . " \\
& 20 . " \\
& 22 . " \\
& 23 . " \\
& 24 . " \\
& 26 . " \\
& 28 . " \\
& 30 .\end{aligned}$ & $\begin{array}{c}\mathrm{g} \\
1970 \\
1753 \\
1743 \\
1717 \\
1693 \\
1690 \\
1513 \\
1390 \\
1280 \\
1195 \\
1105 \\
1008 \\
960 \\
-\end{array}$ & $\begin{array}{c}\mathrm{g} \\
2958 \\
2910 \\
2901 \\
2830 \\
2776 \\
2750 \\
2505 \\
2350 \\
2218 \\
2092 \\
1997 \\
1898 \\
1817 \\
1745 \\
1660 \\
1560\end{array}$ & $\begin{array}{r}177 \mathrm{~g}=10,5 \% \\
123 \%=7,3 \% \\
110 \%=6,5 \% \\
90 \%=5,3 \% \\
95 \%=5,6 \% \\
97 \%=5,7 \% \\
48 \%=2,8 \%\end{array}$ & 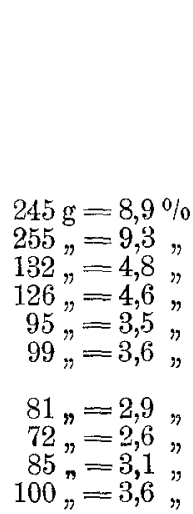 & $\begin{array}{l}\mathrm{ccm} \\
700 \\
565 \\
695 \\
575 \\
410 \\
750 \\
260 \\
150 \\
140 \\
100 \\
100 \\
100\end{array}$ \\
\hline
\end{tabular}

1) W. Swirski, Über das Verhalten des festen Magendarminhaltes bei absoluter 
gefüllten Magen behalten und auch der Aufenthalt des Speisebreies bzw. des Kotes im Darm ausserordentlich lange währt. Ausserdem fressen, wie Swirski ${ }^{1}$ ) gezeigt hat, die Kaninchen während der Inanition ihren Kot, so dass schon aus diesem Grunde mit den Kotanalysen nichts anzufangen wäre. Andererseits nahmen wir davon

Kaninchen Nr. 13 und 14.

\section{Serie III.}

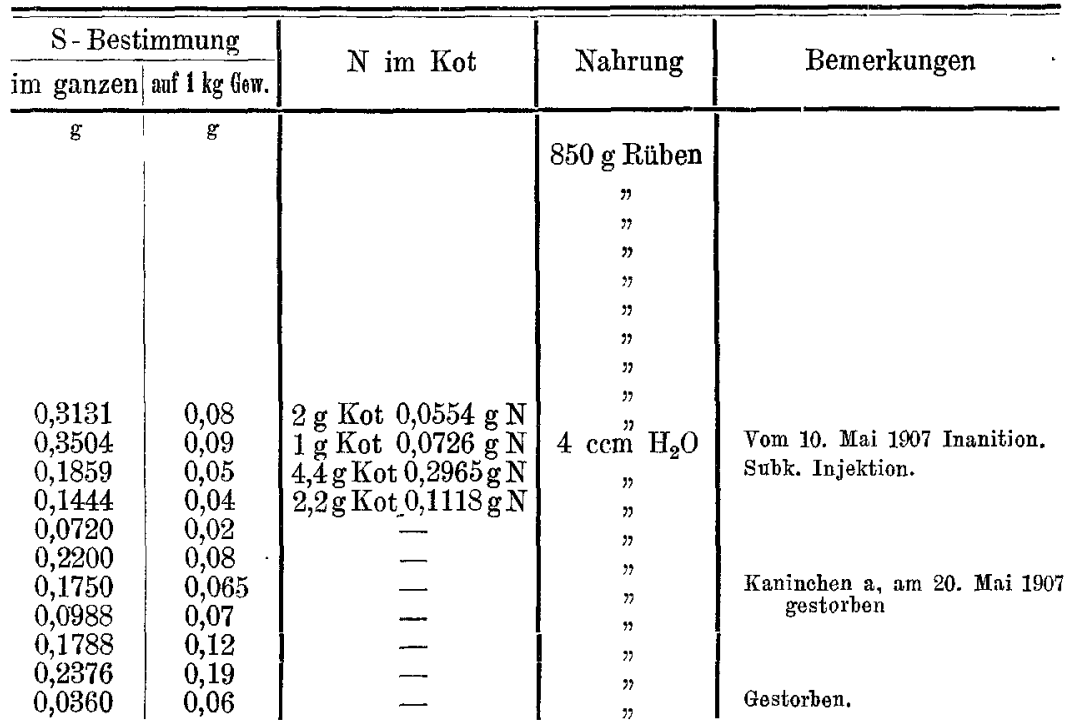

Kaninchen Nr. 15 und 16.

\begin{tabular}{|c|c|c|c|c|c|}
\hline \multicolumn{2}{|c|}{ N - Bestimmung } & \multicolumn{2}{|c|}{ S-Bestimmung } & \multirow{2}{*}{$\mathrm{N}$ im Kot } & \multirow{2}{*}{ Bemerkungen } \\
\hline iill ganzon & anf $1 \mathrm{~kg}$ Gewicht & inl ganzen & auf $1 \mathrm{~kg}$ Gewicht & & \\
\hline $\begin{array}{c}g \\
1,4513 \\
- \\
- \\
1,5040 \\
2.1706 \\
3,4255 \\
3.5994 \\
3,3630 \\
2,7888 \\
3,7625 \\
4,3680 \\
\\
2,6680 \\
2,0750 \\
1,8880 \\
1,5960\end{array}$ & $\begin{array}{l}0,34 \\
0,49 \\
0,85 \\
0,97 \\
0,96 \\
0,85 \\
1,21 \\
1,50 \\
1,59 \\
1,19 \\
1,14 \\
1,02\end{array}$ & $\begin{array}{l}0,2903 \\
0,4256 \\
02449 \\
0,1722 \\
0,1003 \\
0,1978 \\
0,2200 \\
0,2562 \\
\\
0,2088 \\
0,1125 \\
0,1120 \\
0,1316\end{array}$ & $\begin{array}{l}0,06 \\
0,10 \\
0,06 \\
0,05 \\
0,03 \\
0,06 \\
0,07 \\
0,09 \\
0,09 \\
0.06 \\
0,07 \\
0,08\end{array}$ & $\begin{array}{l}10 \mathrm{~g} \text { Kot } 0,4171 \mathrm{~g} \mathrm{~N} \\
6,5 \mathrm{~g} \text { Kot } 0,1580 \mathrm{~g} \mathrm{~N} \\
6,4 \mathrm{~g} \text { Kot } 0,4646 \mathrm{~g} \mathrm{~N} \\
7,2 \mathrm{~g} \text { Kot } 0,3701 \mathrm{~g} \mathrm{~N} \\
8,2 \mathrm{~g} \text { Kot } 0,3575 \mathrm{~g} \\
2,6 \mathrm{~g} \text { Kot } 0,2184 \mathrm{~g} \mathrm{~N} \\
-\end{array}$ & $\begin{array}{l}850 \mathrm{~g} \text { Rüben. } \\
\text { " } \\
\text { 3ccm 10\%o igen Alkoh. } \\
\text { Subkutane Injektion bis } \\
\text { ztum Tode. }\end{array}$ \\
\hline
\end{tabular}

Karenz der Kaninchen. Arch. f. exper. Pathol. und Pharmak: Bd. 48 S. 282. 1902. 
Tabelle XV.

\begin{tabular}{|c|c|c|c|c|c|}
\hline \multirow{2}{*}{$\underset{1907}{\text { Datum }}$} & \multicolumn{2}{|c|}{ Körpergewicht } & \multicolumn{2}{|c|}{ Gewichtsverlust in $\mathrm{g}$ und $\%$} & \multirow{2}{*}{$\begin{array}{l}\text { Urin- } \\
\text { menge }\end{array}$} \\
\hline & $a$ & $\mathbf{b}$ & $\mathrm{a}$ & b & \\
\hline $\begin{aligned} \text { 30. } & \text { April } \\
6 . & \text { Mai } \\
7 . & " \\
8 . & " \\
9 . & " \\
10 . & " \\
12 . & " \\
14 . & " \\
16 . & " \\
18 . & " \\
20 . & " \\
21 . & " \\
22 . & " \\
24 . & " \\
26 . & " \\
28 . & " \\
30 . & "\end{aligned}$ & $\begin{array}{c}g \\
2498 \\
2405 \\
2410 \\
2408 \\
2313 \\
2418 \\
2183 \\
2090 \\
1980 \\
1902 \\
1815 \\
1740 \\
1670 \\
1592 \\
1515 \\
1365\end{array}$ & $\begin{array}{c}\mathrm{g} \\
1955 \\
1993 \\
1997 \\
1999 \\
1970 \\
2002 \\
1845 \\
1732 \\
1610 \\
1460 \\
1308 \\
1260 \\
- \\
- \\
- \\
-\end{array}$ & $\begin{array}{r}235 \mathrm{~g}=9,7 \% \\
93 "=3,8 \% \\
110 \%=4,5 " \\
78 "=3,2 " \\
87 "=3,6 " \\
75 \%=3,1 \% \\
70 "=2,9 " \\
78 "=3,2 " \\
77 "=3,2 " \\
150 "=6,2 "\end{array}$ & $\begin{array}{r}157 \mathrm{~g}=7,8 \% \\
113 \%=5,6 \% \\
122 \%=6,1 \% \\
150 \%=7,5 \% \\
152 \%=7,5 \% \\
48 \%=2,4 \%\end{array}$ & $\begin{array}{c}\text { cem } \\
450 \\
590 \\
605 \\
585 \\
455 \\
610 \\
290 \\
150 \\
160 \\
165 \\
165 \\
\\
75 \\
50 \\
55 \\
50 \\
90\end{array}$ \\
\hline
\end{tabular}

Abstand, den Tieren einen Maulkorb anzulegen, um sie am Kotfressen zu hindern, da wir glaubten, dass der Zwang eines solchen ebenfalls einen und dazu nicht kontrollierbaren Finfluss auf die Tiere ausüben könne. Zwei der Kaninchen dienten als Kontrolltiere and erhielten $4 \mathrm{ccm}$ Wasser, zwei weitere Tiere bekamen $3 \mathrm{ccm}$, und die beiden letzten $5 \mathrm{ccm} 10 \%$ igen Alkohols. Die Einverleibung der Flüssigkeit geschah auf subkutanem Wege, da die Beibringung solch kleiner Flüssigkeitsmengen per os mit Hilfe der Schlundsonde umständlich ist und die Dosierung ganz ungenau wird. Auch hatten uns die früheren Versuche gezeigt, dass $10 \%$ iger Alkohol offenbar schwere Alterationen der Magenschleimhaut hervorbringen kann.

Wie wir später sehen werden, lassen sich bei der gewählten Versuchsanordnung noch keine bindenden Schlüsse auf den Stoffumsatz ziehen. Der Hauptfehler ist der, dass zwei Tiere in einen Käfig zusammengebracht wurden und es sich dann kaum übersehen lässt, welchen Anteil jedes der Tiere an den Ausscheidungen nimmt. Auch die Berechnung der Ausfuhr des Stickstoffes und des Schwefels auf $1 \mathrm{~kg}$ Körpergewicht der Versuchstiere gibt keine klaren Resultate, eine Berechnung, die sonst wohl, wie auch die späteren Versuchsreihen beweisen, ihre Berechtigung hat. Bei vollkommener Inanition, wie sie in unseren Versuchen vorliegt, muss ein grösseres Tier mit höherem Anfangsgewicht beispielsweise mehr Stickstoff ausscheiden, da es mehr Eiweiss zersetzen wird. Wir sind uns aber wohl be- 
Kaninchen Nr. 17 und 18.

\begin{tabular}{|c|c|c|c|c|c|}
\hline \multicolumn{2}{|c|}{$\mathrm{N}$ - Bestimmung } & \multicolumn{2}{|c|}{ S - Bestimmung } & \multirow{2}{*}{$\mathrm{N}$ im Kot } & \multirow{2}{*}{ Bemerkungen } \\
\hline im ganzen & anf $1 \mathrm{~kg}$ Gewicht & im ganzen & auf $1 \mathrm{~kg}$ Gewicht & & \\
\hline $\mathrm{g}$ & $\mathrm{g}$ & $\mathrm{g}$ & $\mathrm{g}$ & & $850 \mathrm{~g}$ Rüben. \\
\hline 1,4463 & 0,33 & & & & $\eta$ \\
\hline 1,5444 & 0,36 & 0,2078 & 0,049 & & $n$ \\
\hline 1,6268 & 0,37 & 0,3976 & 0,09 & $15 \mathrm{~g}$ Kot $0,1985 \mathrm{~g} \mathrm{~N}$ & $5 \mathrm{ccm} 10 \%$ igen Alkoh. \\
\hline 1,8266 & 0,45 & 0,2379 & 0,06 & $1,5 \mathrm{~g} K \mathrm{Kot} 0,0277 \mathrm{~g} \mathrm{~N}$ & \\
\hline 2,5320 & 0,66 & 0,1680 & 0,04 & - & \\
\hline 4,5249 & 1,26 & 0,2226 & 0,06 & - & \\
\hline 5,5900 & 1,66 & 0,3588 & 0,11 & - & \\
\hline 5,4562 & 1,74 & 0,3825 & 0,12 & - & $4 \mathrm{~m} 7 \mathrm{Vaj} 7007$ \\
\hline 2,4000 & 1,01 & 0,1530 & 0,06 & - & \\
\hline 1,6750 & 1,00 & 0,1175 & 0,07 & - & \\
\hline 1,8000 & 1,13 & 0,1075 & 0,07 & - & \\
\hline 1,8630 & 1,23 & 0,1188 & 0,08 & - & \\
\hline 3,0155 & 2,21 & 0,2146 & 0,16 & - & Gestorben. \\
\hline
\end{tabular}

wusst, dass die Mehrausscheidung mit dem Körpergewicht nicht parallel gehen wird. So ungenau diese Berechnung auch immer sein wird, so gibt sie uns doch einen Massstab des Vergleiches, der sonst ausgeschlossen wäre, wenn die Tiere nicht genau das gleiche Anfangsgewicht hätten, von anderen Bedingungen ganz abgesehen.

Bevor die Hungerperiode angefangen wurde, liessen wir die Tiere in der dritten Versuchsreihe längere Zeit bei gleichem Futter und beobachteten sie sorgfältig bezüglich ibres Verhaltens, ihres Körpergewichts usw. Erst wenn die Tiere an ihren Aufenthalt im Käfig und an ihr zugewogenes Futter gewöhnt waren und sie sich auf ein gleiches Körpergewicht eingestellt hatten, wurde ihr Urin analysiert und dann mit der zweiten Periode des Versuches, der Inanition, begonnen. Die Einzelheiten der Versuchsergebnisse sind aus den Tabellen XIII, XIV und XV zu ersehen, während die analytischen Belege am Schluss der Arbeit angeführt werden sollen.

Die Tiere, welche nur $4 \mathrm{ccm}$ Wasser erhalten hatten, besassen also ein Anfangsgewicht von 1745 bzw. $2025 \mathrm{~g}$ und lebten nach Aussetzen der Nabrung 10 und 18 Tage. Die Tiere, denen $3 \mathrm{ccm}$ 10\% igen Alkohols beigebracht wurden, und welche ein Körpergewicht von 1690 bzw. $2750 \mathrm{~g}$ vor Beginn der Inanition aufwiesen, lebten 13 und 20 Tage. Kaninchen Nr. 17 und Nr. 18 mit einem Anfangsgewicht von $2418 \mathrm{bzw} .2002 \mathrm{~g}$ blieben bei täglicher Injektion von $5 \mathrm{ccm} 10 \%$ igen Alkohols 20 und 11 Tage am Leben. In Tabellenform ist die Übersicht über diese Verhältnisse bedeutend erleichtert. 
Tabelle XVI.

Übersichtstabelle.

\begin{tabular}{|c|c|c|c|c|}
\hline $\begin{array}{c}\text { Kaninchen } \\
\text { Nr. }\end{array}$ & $\begin{array}{c}\text { Anfängliches } \\
\text { Körpergew. } \\
\mathrm{g}\end{array}$ & $\begin{array}{l}\text { Wasser bzw. } \\
\text { Alkohol während } \\
\text { der Inanition }\end{array}$ & $\begin{array}{c}\text { Lebensdauer } \\
\text { Tage }\end{array}$ & $\begin{array}{l}\text { Körpergewichts- } \\
\text { verlust } \\
\text { in } \mathrm{g} \text { und } \%\end{array}$ \\
\hline $\begin{array}{l}13 \\
14 \\
15 \\
16 \\
17 \\
18\end{array}$ & $\begin{array}{l}1745 \\
2025 \\
1690 \\
2750 \\
2418 \\
2002\end{array}$ & $\begin{array}{l}(2,3) 4 \mathrm{ccm} \mathrm{H}_{2} \mathrm{O} \\
(1,9) 4 \mathrm{ccm} \mathrm{H}_{2} \mathrm{O} \\
(1,8) 3 \mathrm{ccm} \text { Alkohol } \\
(1,1) 3 \mathrm{ccm} \text { Alkohol } \\
(2,07) 5 \mathrm{~cm} \text { Alkohol } \\
(2,4) 5 \mathrm{ccm} \text { Alkohol }\end{array}$ & $\begin{array}{l}10 \\
18 \\
13 \\
20 \\
20 \\
11\end{array}$ & $\begin{aligned} 627 \mathrm{~g} & =35,9 \% \\
790 \% & =39,0 \% \\
730 " & =43,2 \% \\
1190 " & =43,3 \% \\
1059 " & =43,5 \% \\
742 " & =37,1 \%\end{aligned}$ \\
\hline
\end{tabular}

Bei strenger Kritik lassen sich die Tiere nicht ohne weiteres miteinander vergleichen. Kaninchen Nr. 16 und Nr. 17 sind wesentlich schwerer als die übrigen Tiere; daher ist bei den mit gleicher Alkoholgabe behandelten Tieren die Dosis auf Körpergewicht berechnet keineswegs dieselbe, und andererseits ist es eine Erfahrungstatsache, dass die grösseren Tiere länger leben, weil sie sich unter günstigeren Bedingungen befinden. So haben sie z. B. eine relativ kleinere Oberfläche und infolgedessen eine wesentlich geringere Wärmeabgabe. Wenn man die Alkoholdosen auf Kilogramm Tier berechnet, so erhält man die Zahlen, welche in der dritten Zahlensäule eingeklammert sich finden.

Vergleicht man nun zunächst die Tiere mit annähernd gleichem Körpergewicht miteinander, so dürfen einerseits Kaninchen Nr. 13 mit Nr. 15 und andererseits Kaninchen Nr. 14 mit Nr. 18 in Parallele gesetzt werden, und da zeigt es sich denn, dass Kaninchen Nr. 13, ein "normales Hungertier", wie wir von jetzt ab die Tiere nennen wollen, welchen nur Wasser verabfolgt wurde, trotz eines höheren Anfangsgewichtes 3 Tage weniger lebte als das "3-ccm-Alkoholtier". Das fällt noch schwerer ins Gewicht, wenn man bedenkt, dass Kaninchen Nr. 13 mehr Wasser erhalten hat als Kaninchen Nr. 15; denn es ist bekannt, dass die Tiere oder Menschen relativ länger leben, wenn ihnen Wasser gereicht wird. Es läge auf diese Weise der Gedanke recht nabe, dass der Alkohol bei dieser Dosierung einen günstigen Einfluss auf die Lebensdauer ausgeübt hätte. Interessant ist es auch, die Körpergewichtsabnabme dieser beiden Tiere miteinander zu vergleichen: Nr. 13 nimmt in 10 Tagen bis zum Tode $627 \mathrm{~g}$ ab und Nr. 15 n u r $585 \mathrm{~g}$. 
Stellen wir denselben Vergleich bei den Tieren Nr. 14 und Nr. 18 an, so sehen wir, dass bei nahezu ganz gleichem Anfangsgewicht (23 g Differenz) das "normale Hungertier" 7 Tage länger lebt als das ,5-cem-Alkoholtier", und während Nr. 18 in den 11 Tagen bis zum Tode $742 \mathrm{~g}$ abgenommen hat, beträgt der Körpergewichtsverlust von dem "Normal-Hungertier" in 12 Tagen nur $\mathbf{5} 42 \mathrm{~g}$. Man könnte sich deshalb die Ansicht bilden, dass der 10\% ige

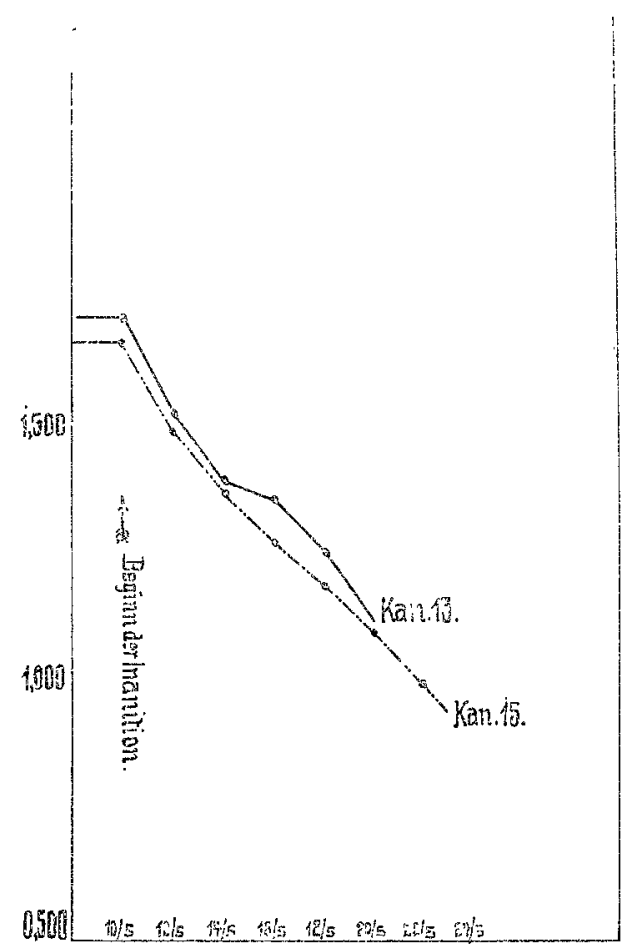

Kurve 1. (Serie III.) Körpergewicht in Gramm.

Alkohol in der Gabe von 5 ccm subkutan bei Nr. 18 schon einen toxischen Einfluss auf die Lebensdauer ausgeübt habe.

Die Tiere Nr. 16 und Nr. 17 müssen wohl oder übel der Betrachtung entfallen, da ihre Anfangsgewichte nur schwer einen Vergleich zulassen. Höchstens könnte man sich denken, dass die Gabe

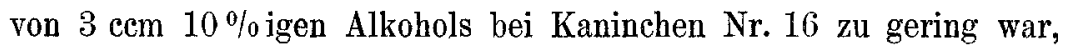
als dass eine günstige Einwirkung auf die Lebensdauer zu beobachten gewesen wäre. Das Kaninchen Nr. 16 wäre dann wie ein Kontrolltier, ein „Normal-Hungertier" zu betrachten. Dann aber würde 
man Kaninchen Nr. 17 mit Tier Nr. 16 vergleichen dürfen, und man würde finden, dass bei Nr. 17 die verabreichte Alkoholgabe von $2,07 \mathrm{~g}$ pro Kilogramm Tier eine günstige Beeinflussung der Lebensdauer zur Folge gehabt hätte, da Nr. 17 ebensolange gelebt hat wie Nr. 16, nämlich 20 Tage, obwohl das Anfangsgewicht um $332 \mathrm{~g}$ zuungunsten von Nr. 17 differierte.

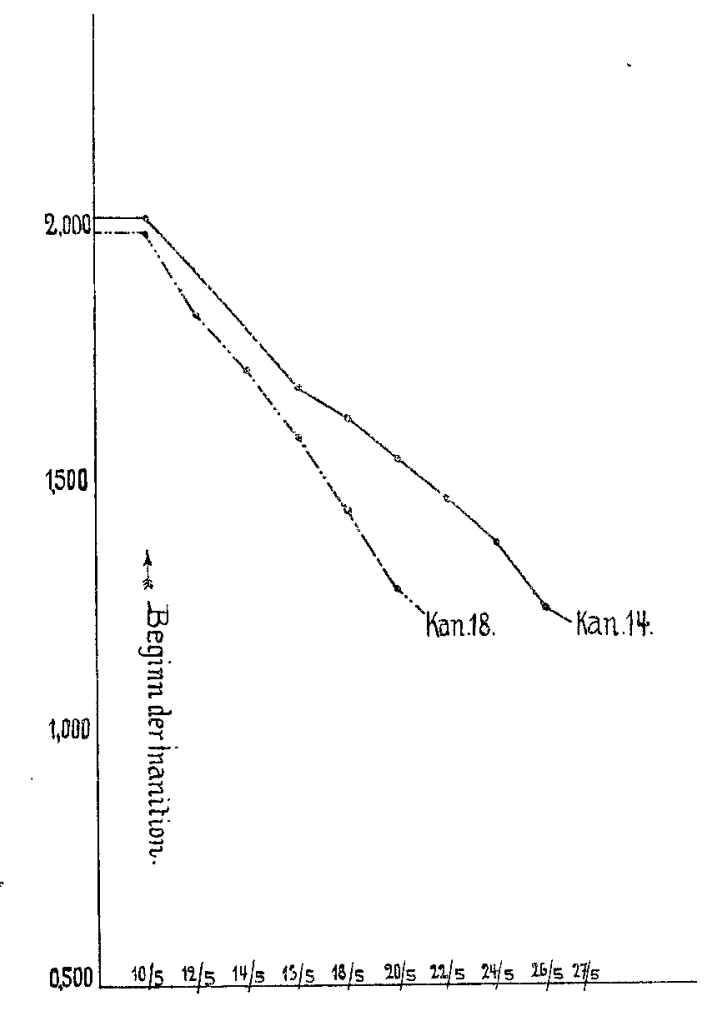

Kurve 2. (Serie III.) Körpergewicht in Gramm.

So zeigt uns diese Versuchsreihe, dass bei passender Dosierung ein günstiger Finfluss des Alkohols auf die Lebensdauer des Kaninehens wahrscheinlich sei. Die Beziehungen des Körpergewichts der Tiere, welche soeben miteinander verglichen wurden, lassen sich zweckmässig in übersichtlicher Form durch Kurve Nr. 1-4 erläutern.

Es fällt zunächst bei den Kurven $5-7$ auf, dass mit Beginn der Inanition die Stickstoffausscheidung um ein weniges unter den Anfangswert sinkt, um alsdann wieder zu steigen. Die Steigerung ist am grössten bei den mit $5 \mathrm{ccm} 10 \%$ igen Alkohols behandelten 
Tieren, am geringsten bei den Tieren, welche täglich $3 \mathrm{ccm}$ Alkohol bekamen. Hier hält sich die Stickstoffausscheidung sogar mehrere Tage annähernd auf gleicher Höhe. Auf deutliche Weise ist die "prämortale" Stickstoffsteigerung zu bemerken, die sich besonders

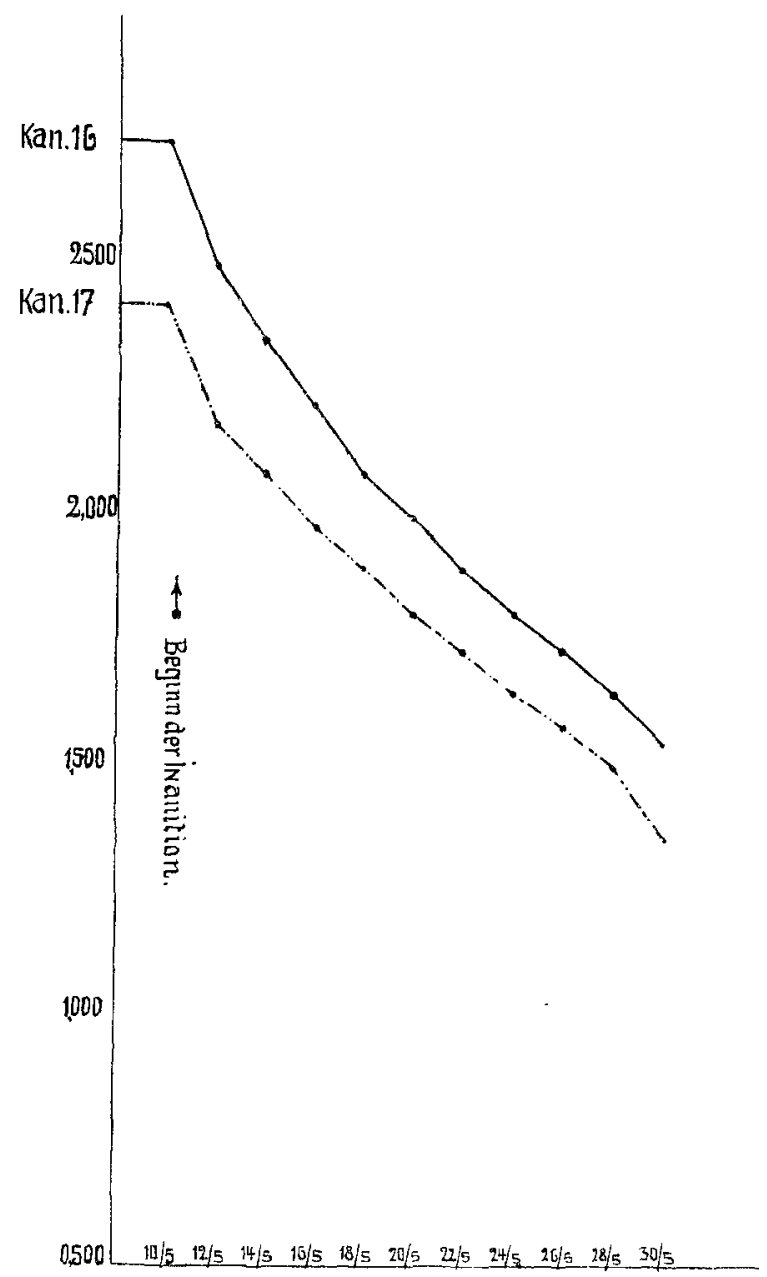

Kurve 3. (Serie III.) Körpergewicht in Gramm.

markant bei den "5-cem-Alkoholtieren" kundgibt, und zwar sowohl beim Tode des ersten wie des zweiten Tieres.

Im allgemeinen parallel mit der Stickstoffausscheidung geht wäbrend der Inanition die Ausscheidung des Schwefels, so dass man den Eindruck gewinnt, dass der Schwefel denselben Ursprung hat 


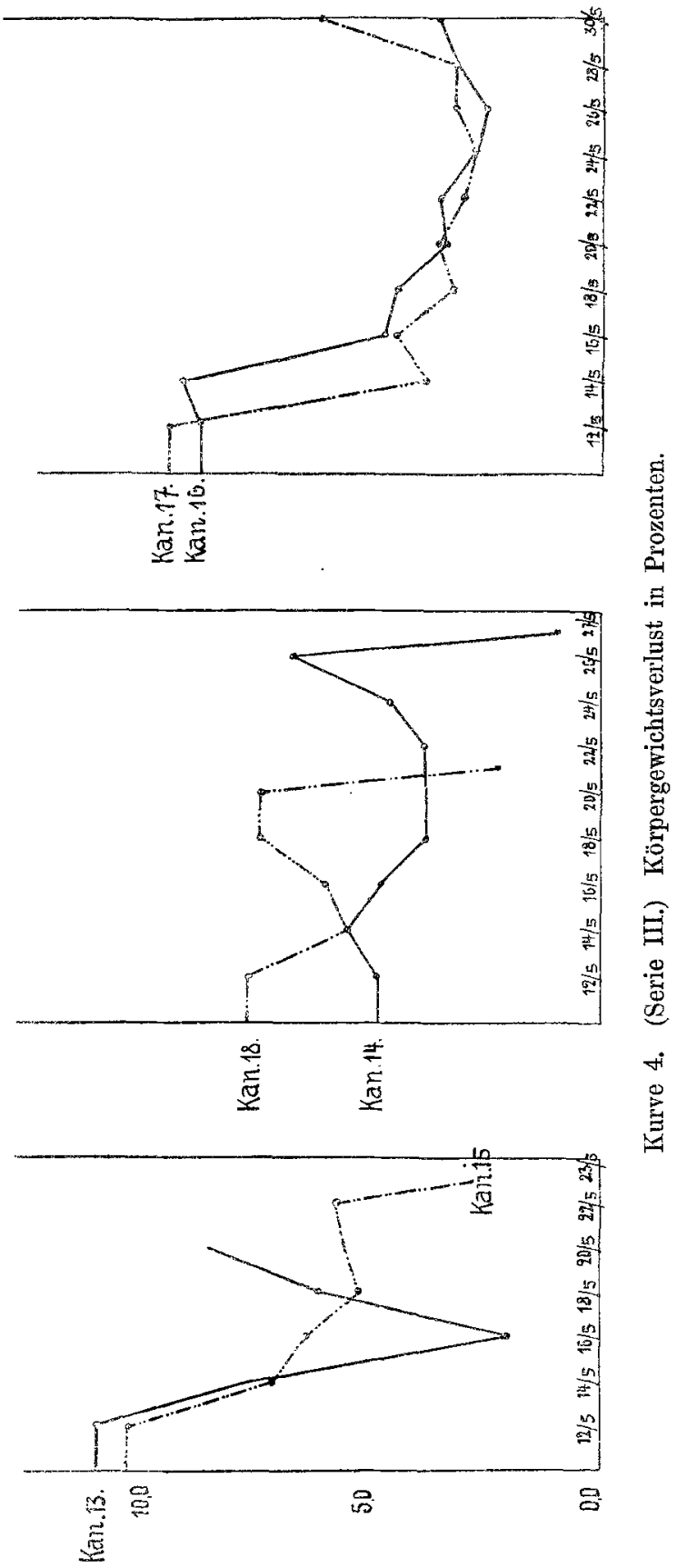


wie der Stickstoff, nämlich vom Eiweiss herstammt. Auffällig, wenn auch längst bekannt, ist die Schwefelausscheidung beim Übergang von der gewöhnlichen Kost zur Inanition. Es rührt dies daher, dass bei voller Ernährung Schwefelverbindungen vom Darm aus resorbiert

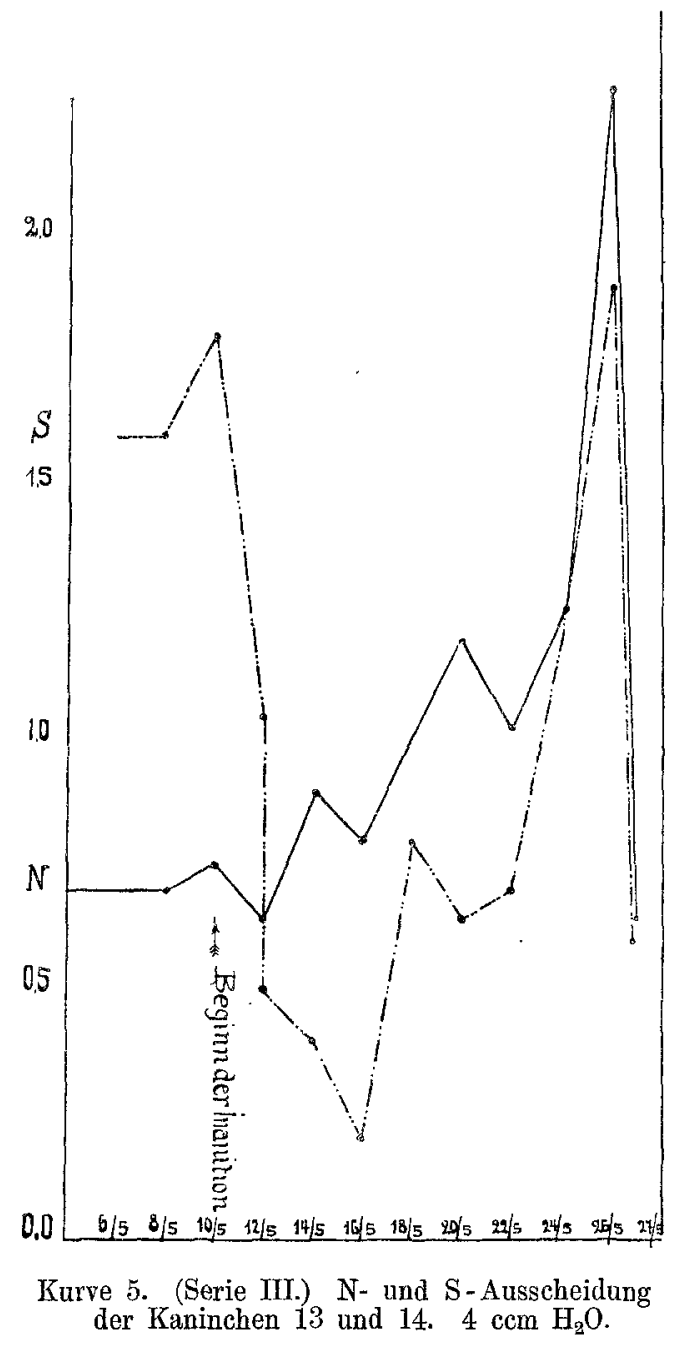

werden und als Ätherschwefelsäure im Urin ausgeschieden werden. Bei der Inanition fällt diese Quelle der Harnschwefelsäure fort, und die Schwefelausscheidung fällt rapide ab. Erwähnenswert ist wohl auch, dass mán bei Betrachtung der Schwefelkurven den Eindruck hat, dass wir durch sie besser als durch die Stickstoffkurven über 
den Eiweisszerfall unterrichtet werden. Schärfer als die „prämortale“ Stickstoffsteigerung ist die „prämortale" Schwefelsteigerung ausgeprägt, und auch sonst sind die Schwankungen der Schwefelkurve, trotz eines gewissen Parallelismus mit der Kurve der Stickstoffausscheidung, prägnanter als bei letzterer.

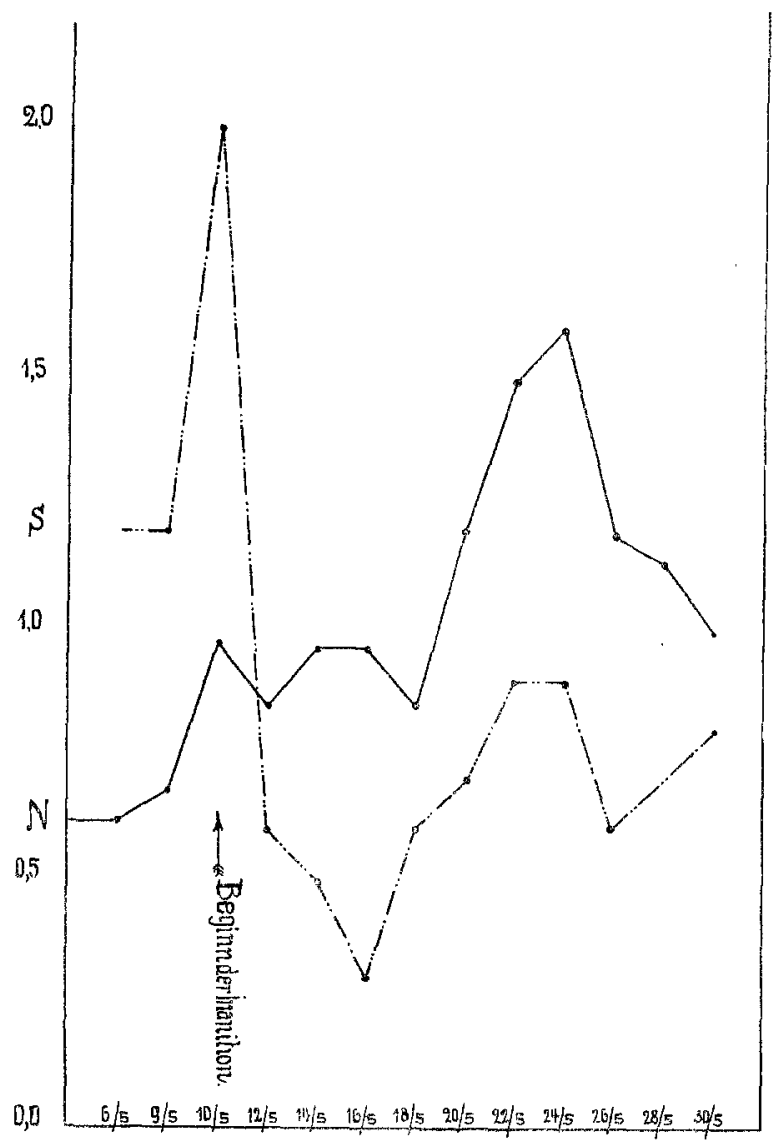

Kurve 6. (Serie III.) N- und S-Ausscheidung der Kaninchen 15 und 16. $3 \mathrm{ccm}$ 10\% igen Alkohols.

Für die Frage der Beeinflussung des Stoffumsatzes durch den Alkohol ist mit den Analysen des Urins in der beschriebenen Versuchsanordnung allerdings nicht viel gewonnen, wenn man auch bei Betrachtung der Kurven sich dem Eindruck nicht entziehen kann, dass die geringeren Gaben ebenso wie auf die Lebensdauer auch auf den Stickstoff- und Schwefelstoffwechsel einen günstigen Einfluss 
Der Einfluss des Alkohols am Hungertier auf Lebensdauer etc. $\quad 315$

haben könnten; denn die Kurve ist gleichmässiger gestaltet. $5 \mathrm{ccm}$ $10 \%$ igen Alkohols scheinen dagegen schon toxisch zu wirken, da die Ausscheidungskurven sehr scharf in die Höhe gehen. Aber, wie schon oben gesagt wurde, mehr als mit einem gewissen Grad von

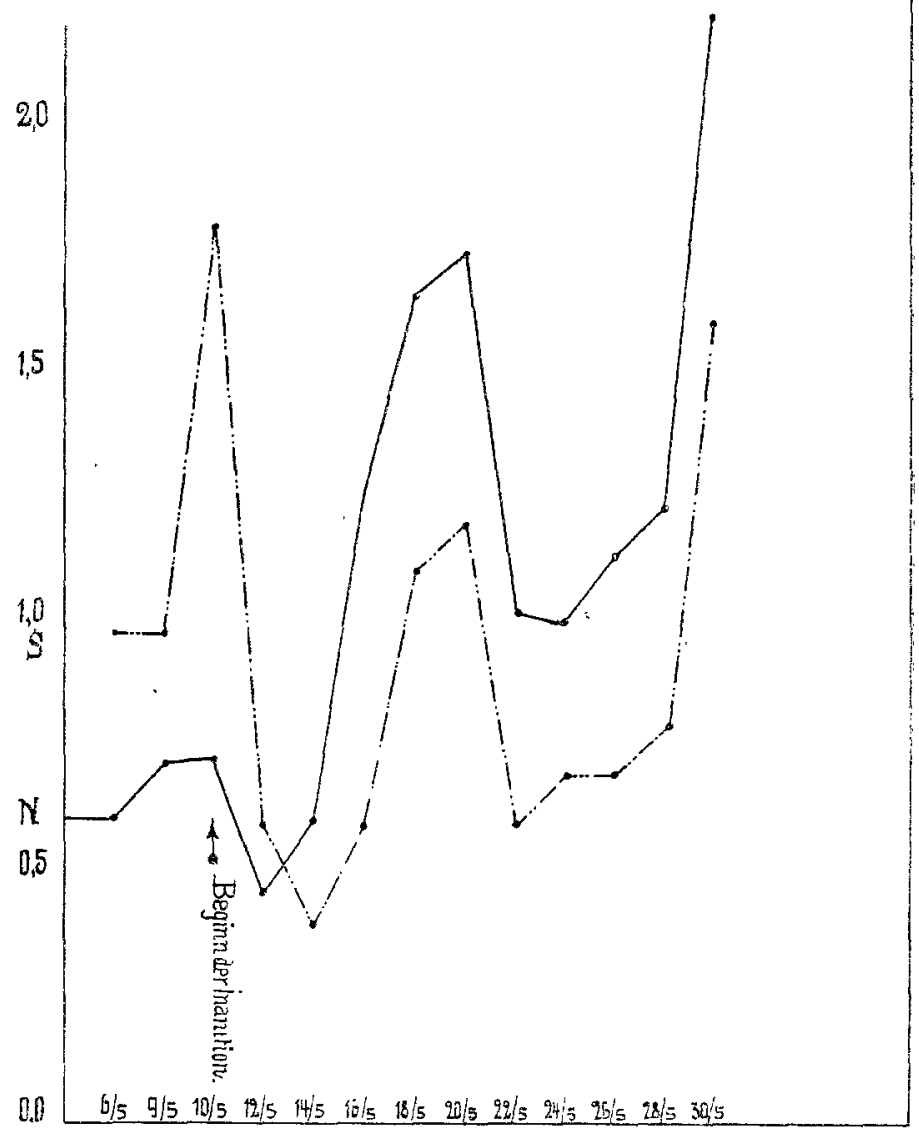

Kurve 7. (Serie III.) N- und S-Ausscheidung der Kaninchen 17 und 18. $5 \mathrm{ccm} 10 \%$ igen Alkohols.

Wabrscheinlichkeit lassen sich diese Dinge nicht behaupten. $\mathrm{Ob}$ diese Wahrscheinlichkeit aber nicht zur Gewissheit werden könnte, das sollte eine neue Versuchsreihe entscheiden, welche im folgenden beschrieben wird.

E. Pflüger, Archiv für Physiologie. Bd. 127. 


\section{Versuchsreihe.}

Die äusseren Bedingungen dieser Versuchsserie waren dieselben wie bei der vorigen. Die Tiere befanden sich in den oben beschriebenen Glaskäfigen; das Körpergewicht wurde alle Tage, während der Inanition alle zwei Tage festgestellt. Der Urin wurde an demselben Tage gemessen und analysiert, indem Stickstoff-, Schwefel- und Phosphorbestimmungen vorgenommen wurden. Je zwei Kontrollanalysen wurden bei jeder der Bestimmungen ausgeführt. Auch im Kot wurde wenigstens eine Stickstoffbestimmung vorgenommen. Der Unterschied zwischen dieser Versuchsreihe und der vorherigen bestand darin, dass jedes Versuchstier in einen besonderen Glaskäfig gesetzt wurde. Im ganzen wurden auch hier ursprünglich sechs Tiere eingestellt; davon schied aber ein Tier infolge besonderer Umstände aus, so dass fünf Tiere übrig blieben. Die Anfangsgewichte der Tiere bei Beginn der Inanition waren von Kaninchen Nr. 191800 g, von Kaninchen Nr. 201742 g, vou Kaninchen Nr. 21 $1975 \mathrm{~g}$, von Kaninchen Nr. $221887 \mathrm{~g}$ und von Kaninchen Nr. 23 2022 g. Kaninchen Nr. 19 diente als Kontrolltier, Nr. 20 und Nr. 21 erhielten $3 \mathrm{ccm}$ bzw. $3,2 \mathrm{ccm}$, Nr. 22 und Nr. $235 \mathrm{ccm}$ bzw. $5,3 \mathrm{ccm}$ $10 \%$ igen Alkohols. Die Dosis war je nach den geringen Unterschieden des Körpergewichtes etwas variiert worden, um den Tieren auch relativ gleiche Alkoholgaben zu verabreichen. Bevor mit den Versuchen begonnen wurde, hielten wir die Kaninchen längere Zeit (3 Wochen) bei gleichem Futter. In den folgenden Tabellen, aus denen sich die Einzelheiten dieses Versuchs leicht entnehmen lassen, sind nur noch die letzten Tage der Fütterung verzeichnet.

(Siehe die Tabellen XVII-XXI [Serie IV] auf Seite 317-319.)

Aus diesen Tabellen geht mit Sicherheit die Tatsache hervor, dass die Tiere Nr. 20 und Nr. 21, welche $3 \mathrm{cem}$ bzw. 3,2 ccm $10 \%$ igen Alkohols pro die erbalten hatten, 3 Tage länger lebten als das Kontrolltier Nr. 19. Und dies ist um so auffallender, als das Kaninchen Nr. 20 leichter war als das Tier Nr. 19. Als das Kontrolltier am zwölften Tage der Inanition starb, hatte es $788 \mathrm{~g}$ $=43 \%$ seines Körpergewichts verloren; die beiden ${ }_{n} 3$-cem-Alkoholtiere" wiesen dagegen in derselben Zeit nur einen Körpergewiıhtsverlust von $613 \mathrm{~g}=34,7 \%$ bzw. $543,5 \mathrm{~g}=26,6 \%$ anf. Sie hatien also $8,3 \%$ bzw. $16,4 \%$ weniger von ihrem Körpergewicht verloren 
Der Einfluss des Alkohols am Hungertier auf Lebensdauer etc.
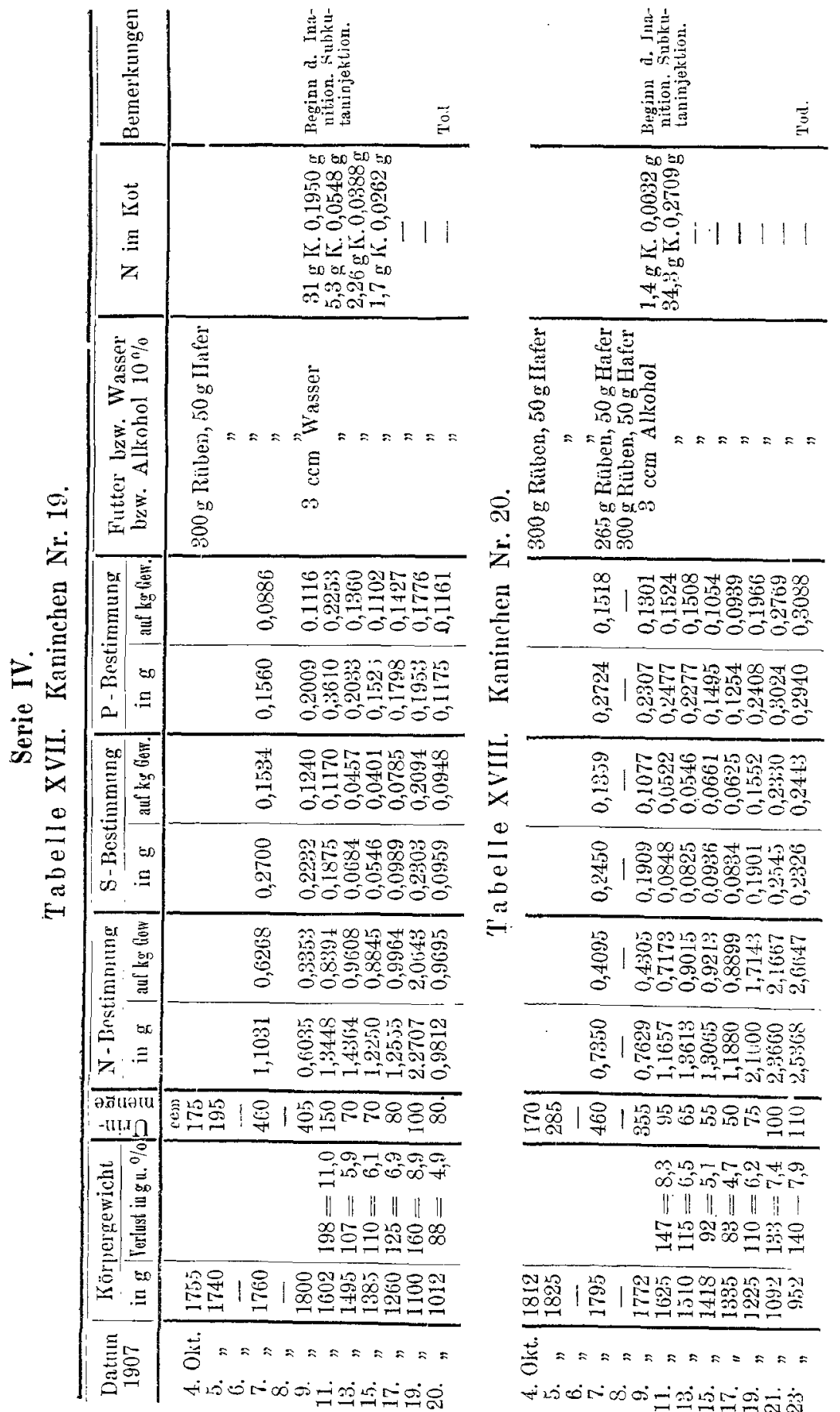

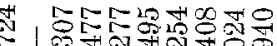

\%

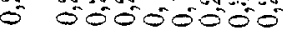

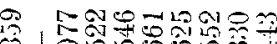

$2101028080 \%$

0 00000000

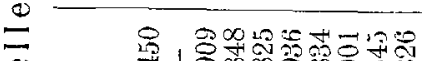

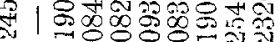

0 0000000-0

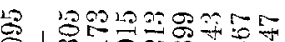

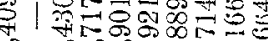

o cóñonion

은

T.

0 of

里息|

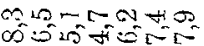

|| || || || || : |

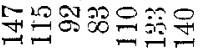

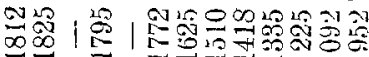

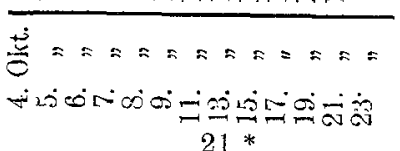



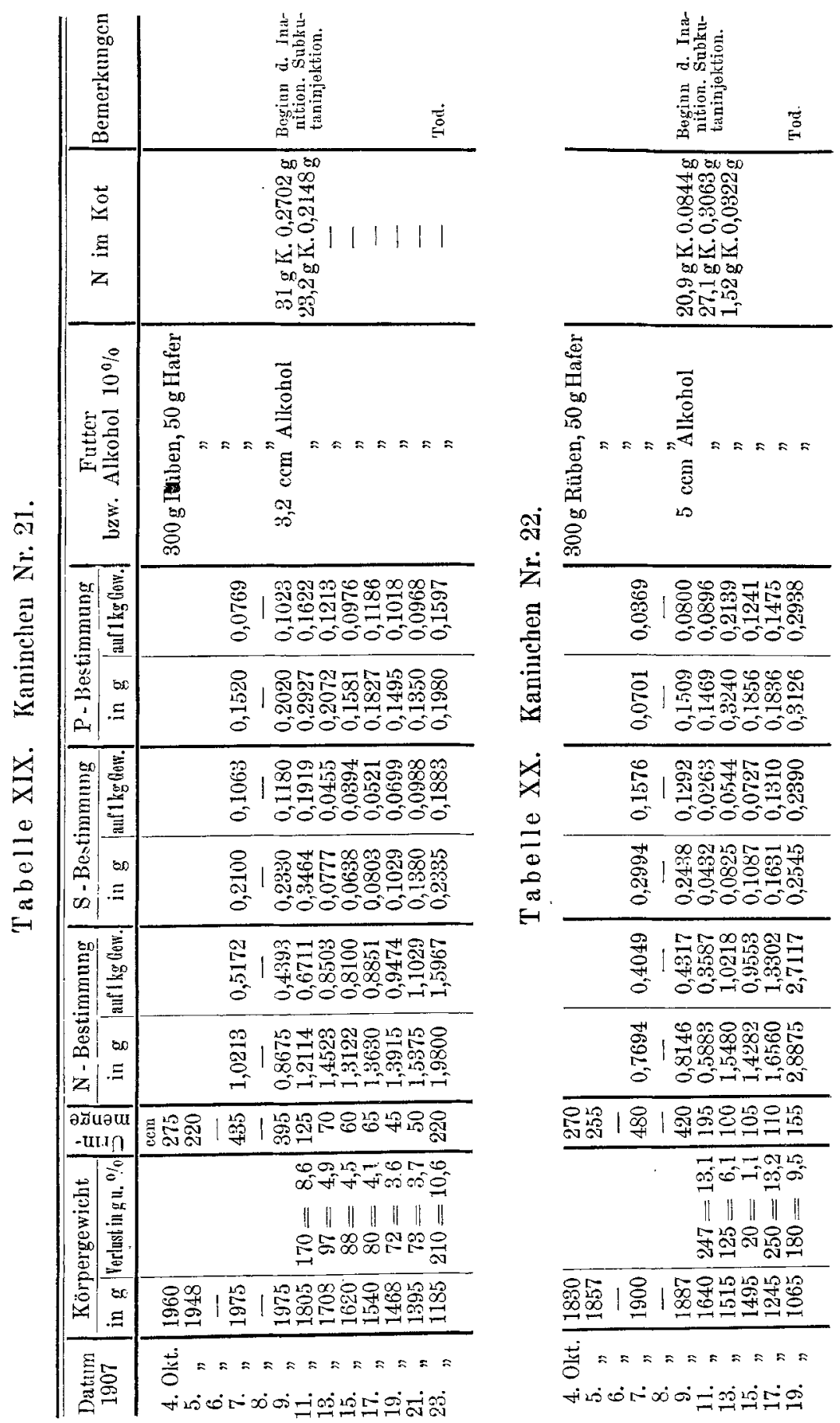
Tabelle XXI. Kaninchen No. 23.

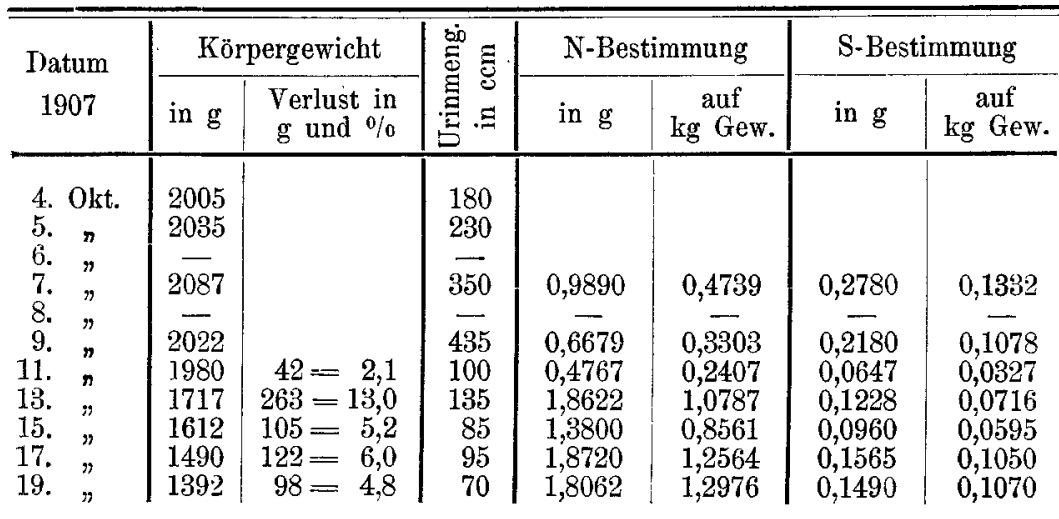

\begin{tabular}{|c|c|c|c|c|c|}
\hline \multirow{2}{*}{$\begin{array}{l}\text { Datum } \\
1907\end{array}$} & \multicolumn{2}{|c|}{ P-Bestimmung } & \multirow{2}{*}{$\begin{array}{l}\text { Futter bzw. } \\
\text { Alkohol } 10 \%\end{array}$} & \multirow{2}{*}{$\begin{array}{c}\mathrm{N} \\
\mathrm{im} \operatorname{Kot}\end{array}$} & \multirow{2}{*}{ Bemerkungen } \\
\hline & in $\mathrm{g}$ & $\begin{array}{c}\text { auf } \\
\mathrm{kg} \text { Gew. }\end{array}$ & & & \\
\hline $\begin{aligned} \text { 4. } & \text { Okt. } \\
5 . & " \\
6 . & " \\
7 . & " \\
8 . & " \\
9 . & " \\
11 . & " \\
13 . & " \\
15 . & " \\
17 . & " \\
19 . & "\end{aligned}$ & $\begin{array}{l}0,2058 \\
\overline{0}, 1657 \\
0,1946 \\
0,2847 \\
0,2070 \\
0,2240 \\
0,1836\end{array}$ & $\begin{array}{l}0,0986 \\
\overline{0,0828} \\
0,0983 \\
0,1658 \\
0,1284 \\
0,1504 \\
0,1319\end{array}$ & $\begin{array}{c}300 \mathrm{~g} \text { Rüben, } 50 \mathrm{~g} \text { Hafer } \\
" \\
270 \mathrm{~g} \text { Riuben, } 45 \mathrm{~g} \text { Hafer } \\
300 \mathrm{~g} \text { Rïben, } 50 \mathrm{~g} \text { Hafer } \\
5,3 \mathrm{ccm} \text { Alkohol } \\
" \\
" \\
" \\
" \\
"\end{array}$ & $\begin{array}{c}19,7 \mathrm{~g} \mathrm{Rot} 0,2039 \mathrm{~g} \\
25,5 \mathrm{~g} \text { Kot } 0,4063 \mathrm{~g} \\
" \\
" \\
" \\
"\end{array}$ & $\begin{array}{l}\text { Beginn der Ina- } \\
\text { nition. Sub- } \\
\text { kutaninjektion }\end{array}$ \\
\hline
\end{tabular}

als das ungefähr gleich schwere Kontrolltier. In Übereinstimmung mit dem Körpergewicht stehen die Ausscheidungen der Urinmengen. Das Kontrolltier schied bis zum Tode $555 \mathrm{ccm}$ Urin aus, die beiden "3-ccm-Alkoholtiere" dagegen produzierten während derselben Zeit je $390 \mathrm{ccm}$, also $165 \mathrm{ccm}$ weniger als das Kaninchen, das die gleiche Menge Flüssigkeit ohne Alkoholzusatz erhalten hatte. Mit der Ausfuhr des Stickstoffes, des Schwefels und des Phosphors verhält es sich ebenso. Kaninchen Nr. 19 scheidet während der Inanition $8,5136 \mathrm{~g}$ Stickstoff aus, während die beiden "3-ccm-Alkoholtiere" $8,3045 \mathrm{~g}$ bzw. $7,4991 \mathrm{~g}$ Stickstoff durch den Urin ausführen; auf Eiweiss berechnet, bedeutet dies bei Nr. 19 einen Eiweissverlust von $53,2100 \mathrm{~g}$, bei Nr. 20 und Nr. 21 einen solchen von nur $51,9031 \mathrm{~g}$ bzw. von $46,8694 \mathrm{~g}$ Eiweiss; also die Kaninchen Nr. 20 und Nr. 21 haben in den 12 Tagen der Inanition gegenüber dem Kontrolltier 1,3069 g bzw. 6,3406 g Eiweiss weniger verbraucht. Die Zahlen 
der Schwefelausscheidung und der Phosphorausfuhr bestätigen im algemeinen diese Ansicht. Kaninchen Nr. 19 scheidet $0,7356 \mathrm{~g}$ : Schwefel während der Inanition aus, das Tier Nr. 20 in derselben Zeit $0,6617 \mathrm{~g}$ Schwefel. Kaninchen Nr. 21 zeigt ungefähr dieselbe Grösse der Schwefelausfuhr, nämlich $0,7398 \mathrm{~g}$, wobei aber zu bedenken ist, dass Tier Nr. 21 etwas grösser war als Nr. 19.

Bezüglich der Phosphorwerte sind den Tahellen folgende Zahlen zu entnehmen: Kaninchen Nr. 19 scheidet 1,2095 g Phosphor, Kaninchen Nr. 20 in derselben Zeit 1,1423 g, Kaninchen Nr. 21 $1,0577 \mathrm{~g}$ Phosphor aus; also auch hier wieder ziemlich erhebliche Unterscbiede zugunsten der mit $3 \mathrm{ccm}$ behandelten Tiere.

\section{Tabelle XXII.}

Übersichtstabelle.

\begin{tabular}{|c|c|c|c|c|c|}
\hline \multirow{2}{*}{$\begin{array}{c}\text { Kaninchen } \\
\text { Nr. }\end{array}$} & \multirow{2}{*}{\multicolumn{2}{|c|}{$\begin{array}{l}\text { Alkoholgabe } \\
\text { lzw. Wasser }\end{array}$}} & \multirow{2}{*}{$\begin{array}{l}\text { Lebensdauer } \\
\text { in Tagen }\end{array}$} & \multicolumn{2}{|c|}{ Körpergewicht } \\
\hline & & & & anfangs & Verlust in $\mathrm{g}$ und $\%$ \\
\hline 19 & $3 \mathrm{ccm}$ & Wasser & 12 & 1800 & $\left\{\begin{array}{l}(700 \mathrm{~g}=38,1 \%) \\
788=43\end{array}\right.$ \\
\hline 20 & $3,0 \quad n$ & Alk. $10 \%$ & 15 & 1772 & $\left\{\begin{array}{r}820 \eta=46,3, \\
(613,==34,7,)\end{array}\right.$ \\
\hline 21 & 3,2 & $\Rightarrow 10 "$ & 15 & 1975 & $\left\{\begin{array}{c}790 "=40 \\
543,5=26,6\end{array}\right.$ \\
\hline $\begin{array}{l}22 \\
23\end{array}$ & $\begin{array}{l}5,0 \% \\
5,3 \%\end{array}$ & $\begin{array}{l}10 " \\
" 10 "\end{array}$ & $\begin{array}{l}11 \\
11\end{array}$ & $\begin{array}{l}1887 \\
2022\end{array}$ & $\begin{array}{l}822 "=43,5 \\
630 "=31,2\end{array}$ \\
\hline
\end{tabular}

\begin{tabular}{c|c|c|c|c}
\hline $\begin{array}{c}\text { Kaninchen } \\
\text { Nr. }\end{array}$ & $\begin{array}{c}\text { N-Ausscheidung } \\
\text { in g }\end{array}$ & $\begin{array}{c}\text { S-Ausscheidung } \\
\text { in g }\end{array}$ & $\begin{array}{c}\text { P-Ausscheidung } \\
\text { in } \mathrm{g}\end{array}$ & $\begin{array}{c}\text { Urinmengen } \\
\text { in ccm }\end{array}$ \\
\hline 19 & $8,5136((7,5441))$ & $0,7356((0,6397))$ & $1,2095((1,0920))$ & $555(435)$ \\
20 & $12,0243(8,3045)$ & $1,0215(0,6617)$ & $1,5875(1,1423)$ & $550(390)$ \\
21 & $10,2479(7,4991)$ & $1,0423(0,7398)$ & $1,3252(1,0577)$ & $635(390)$ \\
22 & 8,1080 & 0,6520 & 1,1530 & 665 \\
23 & 7,4171 & 0,5890 & 1,0939 & 485
\end{tabular}

Die eingeklammerten Zahlen geben den Körpergewichtsverlust, die N-, S- und P-Ausscheidung am zwölftèn Tage der Inanition an, um einen Vergleick mit dem an diesem Tage gestorbenen normalen Hungertiere zu ermöglichen.

Die doppelt eingeklammerten Zahlen bei Kaninchen 19 bedeuten den N-, P-, S-Verlust am elften Tage der Inanition, dem Todestage der 5-ccm-Alkoholtiere.

Auf der Übersichtstabelle sind die Zablen tabellarisch geordnet wiedergergeben und leicht miteinander vergleichbar. Besser als die Tabellen unterrichtet ein Blick auf die Kurven (VIII-XVIII) über den Verlauf des Versuches, die Gewichtsverhältnisse, die Aus- 
scheidung der Urinmengen, des Stickstoffes, des Schwefels und des Phosphors. Auf Kurve VIII sind die Gewichte in Gramm wiedergegeben. Wir sehen, wie das Kontrolltier bei weitem schneller an Gewicht abnimmt als die beiden "3-ccm-Alkoholtiere". Am grössten ist der Unterschied am Todestage des Kaninchens Nr. 19, und man sieht, wie der Abstand der Gewichtskurve der beiden Alkoholtiere sich immer mehr von der des Kontrolltieres entfernt. Die Kurve X,

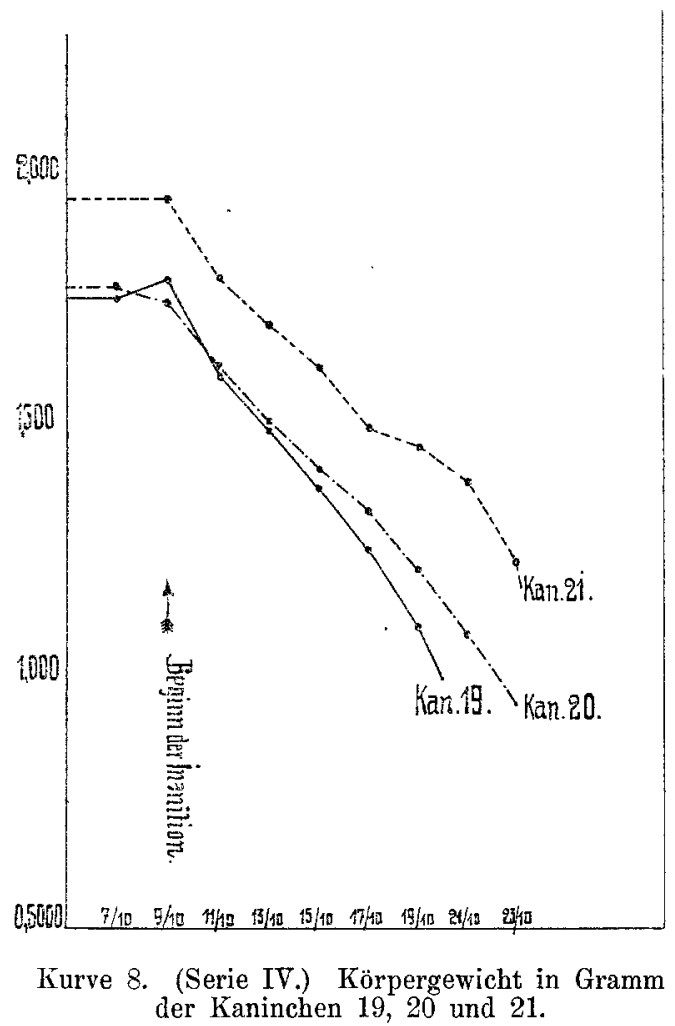

welche die Gewichtsverluste in Prozenten des Anfangsgewichtes wiedergibt, zeigt, wie die Kurve des Verlustes bei Tier Nr. 19 sich eigentlich dauernd oberhalb der anderen Linien bewegt, welche die prozentisehe Gewichtsabnahme der Alkoholtiere andeutet. Auf Kurve XIIa sehen wir, dass die Urinmengen wäbrend der ganzen Dauer der Inanition ungefähr dasselbe Verhältnis zueinander haben. Immer liegt die Kurve von Kaninchen Nr. 19 oberhalb der beiden anderen, ofler, mit anderen Worten, die Diurese bei den „3-ccm-Alkohol- 
tieren" ist geringer als die des Kontrolltieres, was um so bemerkenswerter ist, als die Tiere entweder gleiches Anfangsgewicht aufwiesen oder, wie Kaninchen Nr. 21, schwerer als Kaninchen Nr. 19 gewesen war und demgemäss eigentlich mehr Urin hätte produzieren müssen.

Kurve XIII, XV und XVII zeigen uns die Stickstoff-, Schwefelund Phosphorausscheidung der Tiere, und immer sehen wir, dass bei Beginn der Inanition und der Alkoholdarreichung die Kurvenlinien

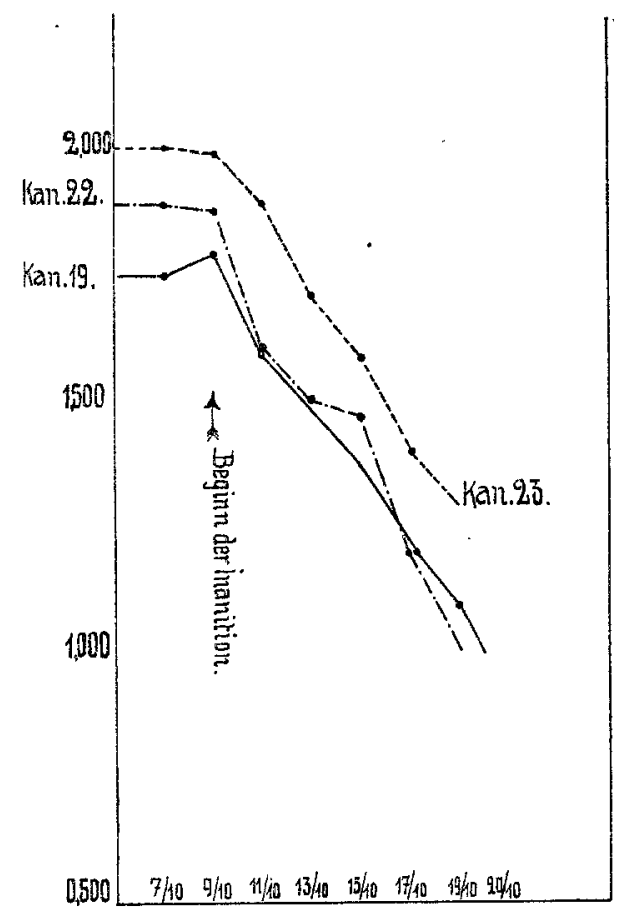

Kurve 9. (Serie IV.) Körpergewicht in Gramm der Kaninchen 19,22 und 28.

von Kaninchen Nr. 20 und Nr. 21 unter die schwarze Linie des "Normal-Hungertieres" sinken, dass also die Verhältnisse immer zuungunsten des Kontrolltieres eine Änderung erfahren.

Ganz andere Eindrücke gewinnt man bei Betrachtung der Tabellen von den Tieren, welche $5 \mathrm{ccm} 10 \%$ igen Alkohols während der Inanition erhielten. Wohl fehlt ein analoges Versuchstier, das 5 ccm Wasser erhalten hätte, aber das Kaninchen Nr. 19, das nur 3 cem Wasser bekommen hatte, kann ohne weiteres 
als Kontrolltier dienen. Kaninchen Nr. 19 lebte 12 Tage, die Alkoholtiere nur 11 Tage; der Gewichtsverlust betrug bei Nr. 19 während dieser 11 Tage $700 \mathrm{~g}=38,1 \%$ des Anfangsgewichtes vou $1800 \mathrm{~g}$. Von den beiden Alkoholtieren verlor Kaninchen Nr. 22 mit $1887 \mathrm{~g}$ Anfangsgewicht $822 \mathrm{~g}=43,5 \%$ und Nr. 23, welches mit einem Anfangsgewicht von $2022 \mathrm{~g}$ in den Versuch kam, $630 \mathrm{~g}$ $=31,2 \%$. Die Ausscheidung des Stickstoffes und des Phosphors verhielt sich ähnlich wie das Körpergewicht, d. h. Kaninchen Nr. 23 sehied etwas weniger aus als das Kontrolltier. Dagegen ist beim

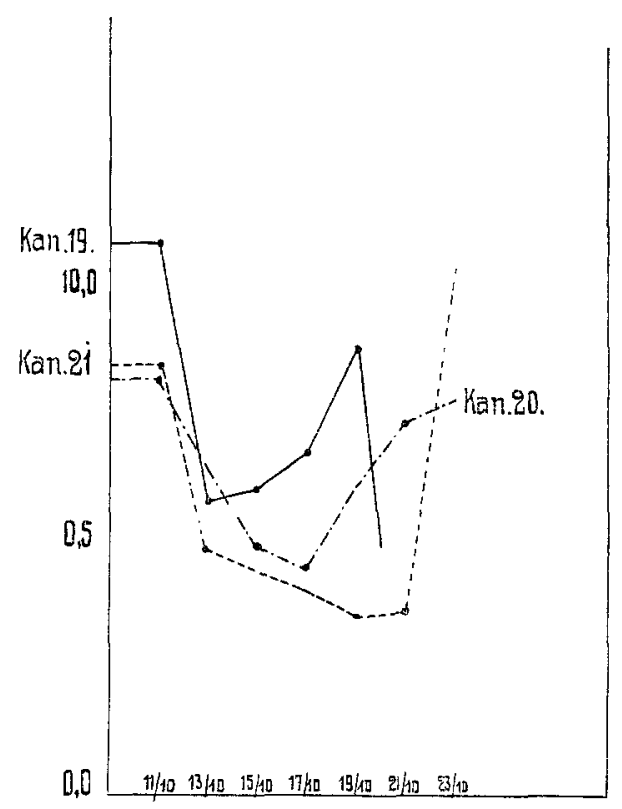

Kurve 10. (Serie IV.) Körpergewichtsverlust in Prozenten der Kaninchen 19, 20 und 21.

Vergleich der Urinmengen und des Schwefels zu bemerken, dass das Kontrolltier die niedrigsten Werte aufweist.

Aus diesen Endergebnissen lässt sich aber noch nicht sagen, wie der Alkohol hier auf den Stoffwechsel eingewirkt hat. Die Kurven dagegen geben hierüber einen genauen Aufschluss. Kurve Nr. XI, welche die Körpergewichte in Gramm verzeichnet enthält, zeigt uns, dass die Linie des Kaninchens Nr. 22 trotz eines höheren Anfangsgewichtes die schwarze Linie des Kontrolltieres schneidet und auch die Kurvenlinie von Kaninchen Nr. 23 konvergiert gegen die des "Normal-Hungertieres", so dass der Unterschied am Todestage des 
Kaninchens Nr. 23 und des Kontrolltieres nur $200 \mathrm{~g}$ beträgt, während er im Anfang des Versuches $250 \mathrm{~g}$ gewesen war. Auch die Aufzeichnung der prozentischen Gewichtsverluste weist darauf hin, dass die Verluste der "5-ccm-Alkoholtiere" im wesentlichen grösser sind als die des Kaninchens Nr. 19.

Das Verhalten der Urinmengen auf Kurve XII b ist äusserst interessant. Vom Tage der Alkoholdarreichung ab liegen die Linien der "5-cem-Alkoholtiere" im allgemeinen höher als die

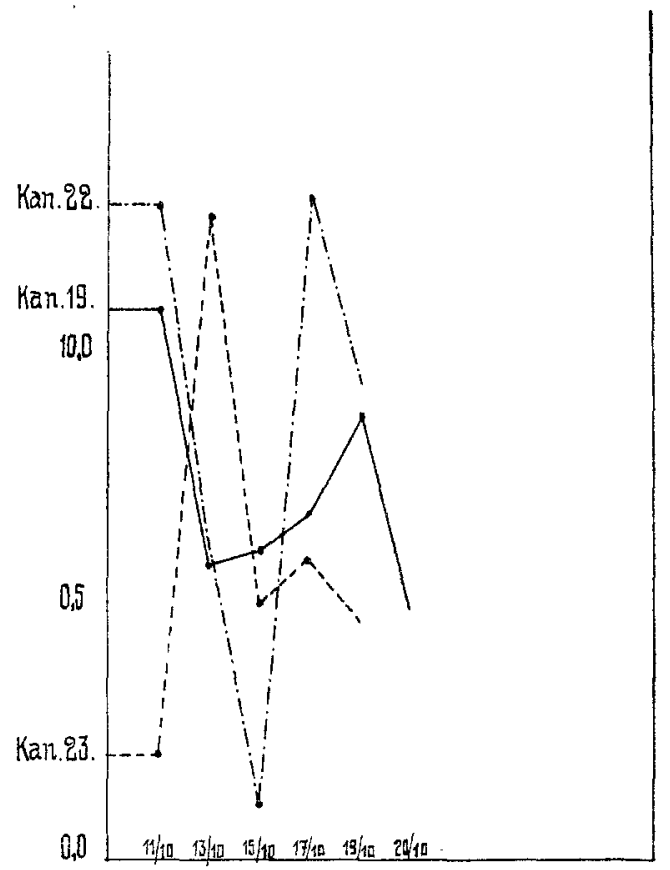

Kurve 11. (Serie IV.) Körpergewichtsverlust in Prozenten der Kaninchen 19, 22 und 23.

Kurvenlinie des Kaninchens Nr. 19. Bei der Gegenüberstellung der beiden graphischen Darstellungen auf Kurve XII a und b kann man sich dem Gedanken nieht verschliessen, dass die oftmals behauptete diuretische Wirkung des Alkohols erst bei der Dosis von 5 cem 10\% igen Alkohols bei unseren Kaninchen zum Vorschein kommt, während $3 \mathrm{cem}$ Alkohol derselben Konzentration diese Wirkung noch nicht besitzen, sondern viel eher eine Einschränkung der Diurese hervorzurufen scheinen. 
Bei aufmerksamer Betrachtung der Kurven XIV, XVI und XVIII, welche die Ausscheidung des Stickstoffes, des Schrefels und des Phosphors aufzeichnen, bemerkt man, dass sich leicht zwei Perioden im Ablauf des Versuches unterscheiden lassen. Die erste Periode
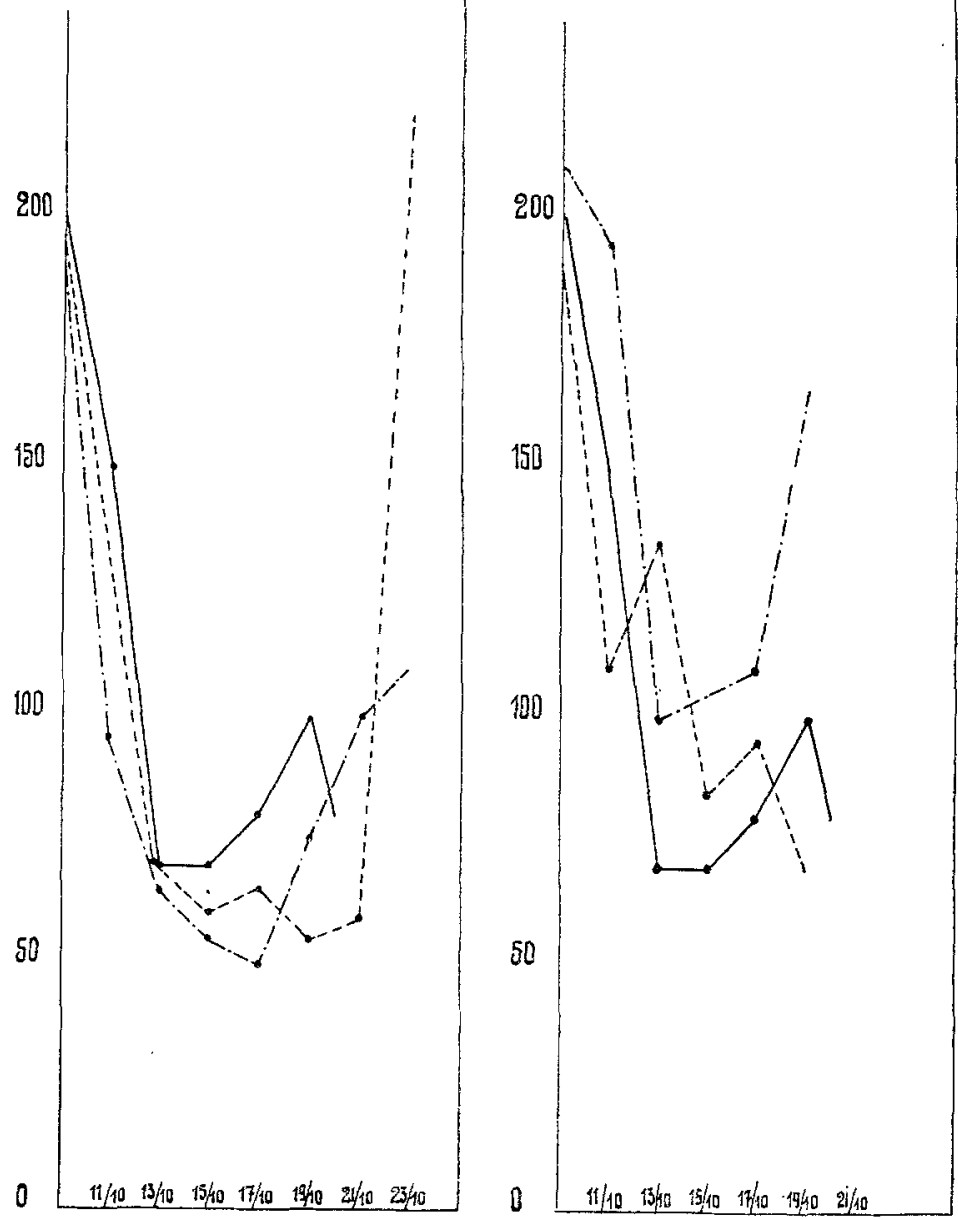

Kurve 12. (Serie IV.) Urinmengen: a) der Kaninchen 19, 20 und 21 , b) der Kaninchen 19,20 und 23.

reicht vom Beginn der Inanition bis zum 13. Oktober 1907, umfasst also einen Zeitraum von 4 Tagen; die andere Periode dauert vom 13. Oktober 1907 bis zum Todle der Versuchstiere. In dem ersten Abschnitt der Kurve liegen die Linien der ,5-ccm-Alkohol- 
tiere" unter der Linie des Kontrolltieres, und erst am 13. Oktober 1907 erheben sich die punktierten Linien über die schwarzen. In der ersten Periode sind die Mengen des ausgeschiedenen Stickstoffes, Schwefels

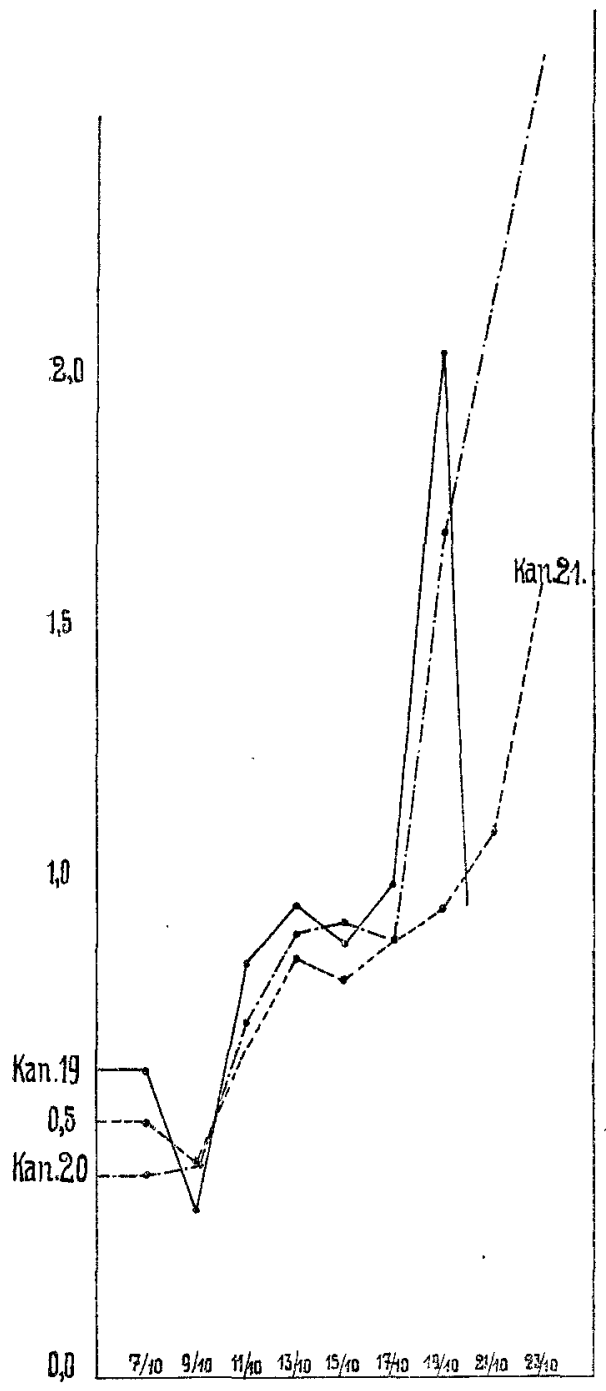

Kurve 13. (Serie IV.) N-Ausscheidung auf Körpergewicht berechnet. Kaninchen 19, 20 und 21 .

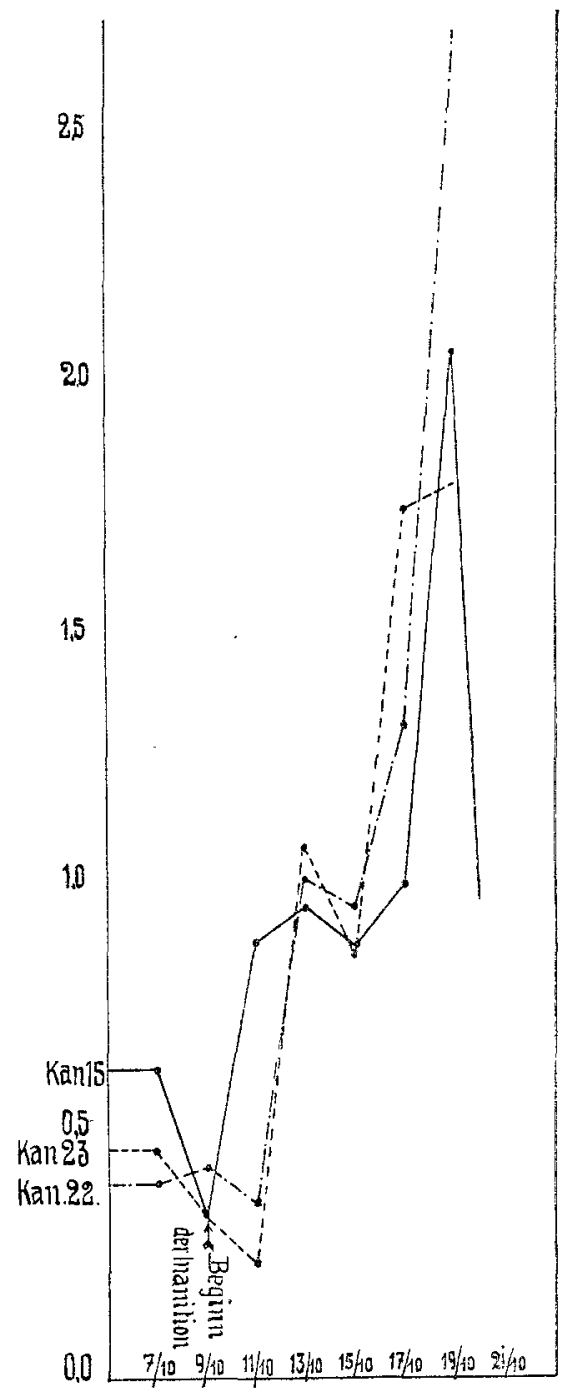

Kurve 14. (Serie IV.) N-Ausscheidung auf Kilogramm Körpergewicht berechnet. Kaninchen 19,22 und 23 .

und Phosphors geringer als beim Kontrolltier; die Tiere Nr. 22 und Nr. 23 sparen da offenbar an Eiweiss. Im zweiten Teil der Periode kehren sich diese Verhältnisse um. 
Man muss angesichts dieser Tatsachen den Eindruck gewinnen, dass der Alkohol bis zum 13. Oktober 1907, also während der ersten 4 Tage der Inanition, einen günstigen Einfluss auf den Stoffumsatz

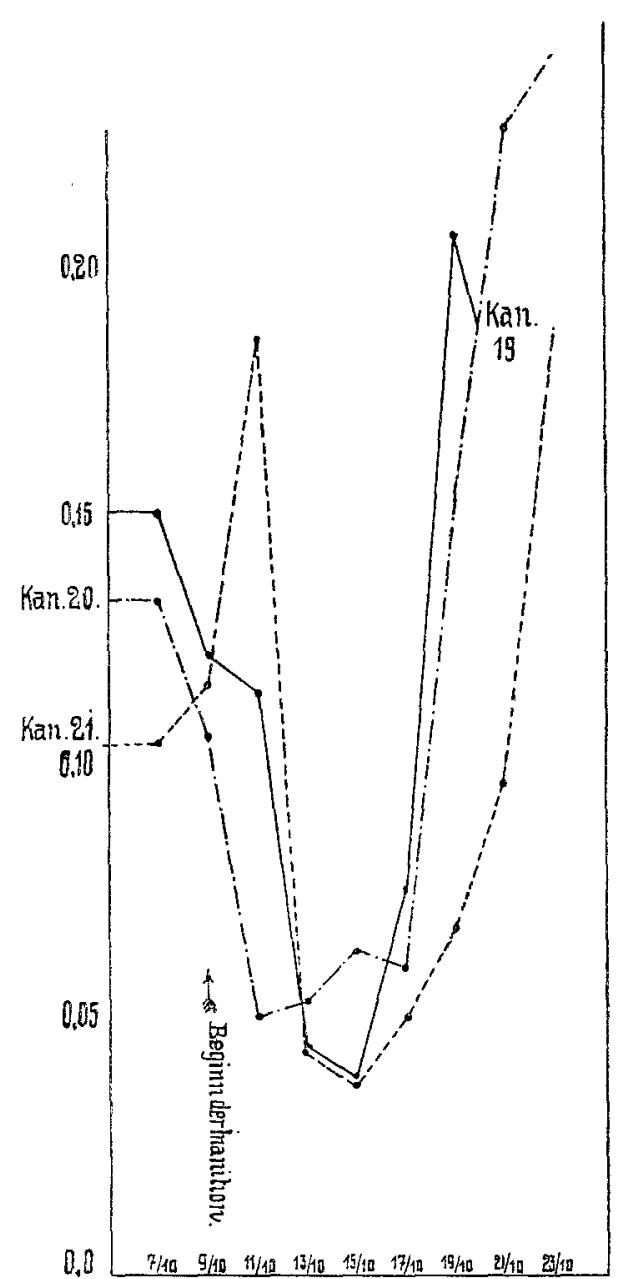

Kurve 15. (Serie IV.) P-Ausscheidung auf Kilogramm Körpergewicht berechnet. Kaninchen 19,20 and 21 .

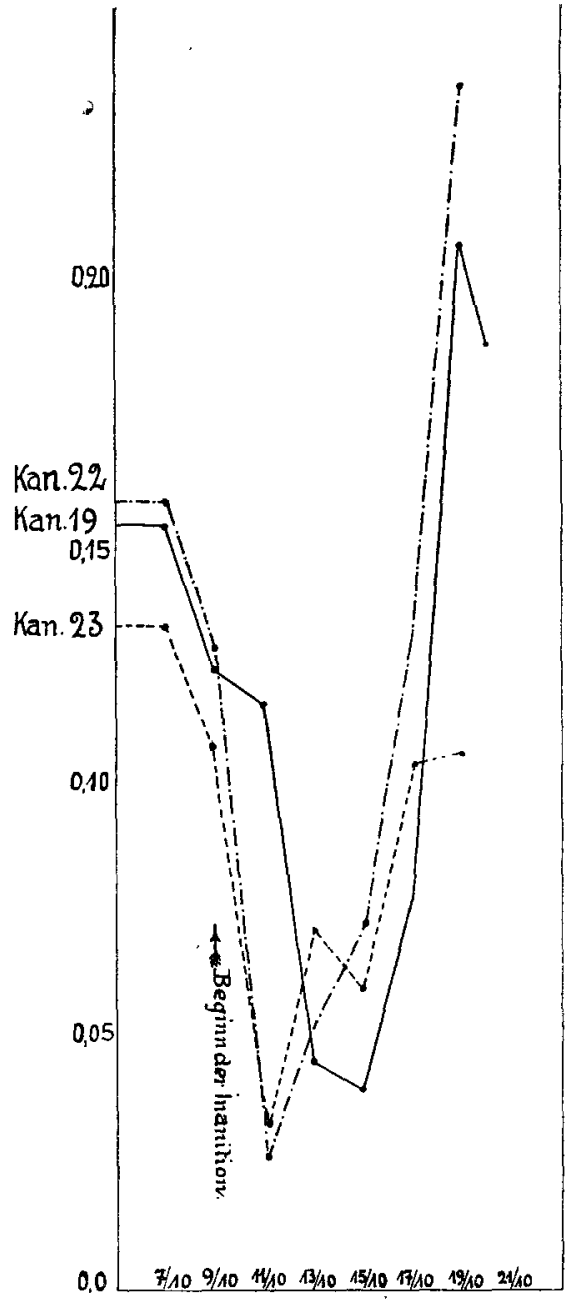

Kurve 16. (Serie IV.) P-Ausscheidung auf Kilogramm Körpergewicht berechnet. Kaninchen 19,22 und 23.

ausgeübt habe, indem Eiweiss geschont wurde, und dass er im zweiten Teil der Versuche toxisch gewirkt hat dadurch, dass er den Eiweissbestand des Organismus angriff, was sich durch eine Steigerung der Ausfuhr von Stickstoff, Schwefel und Phosphor kundgab. Ein 
solches Verhalten wäre auch gar nichts Wunderbares, wenn man bedenkt, dass die tägliche Dosis von 5 cem bzw. $5,3 \mathrm{ccm} 10 \%$ igen Alkohols bei den Tieren im Anfang sicher geringere Wirkungen ausübte, da sie schwerer waren als später bei immer grösserer $\mathrm{Ab}$ nahme des Körpergewichts.

Aus dieser Versuchsreihe lassen sich nun schon viel eher als aus der Serie 3 bindende Schlüsse ziehen. Man muss zugeben, dass

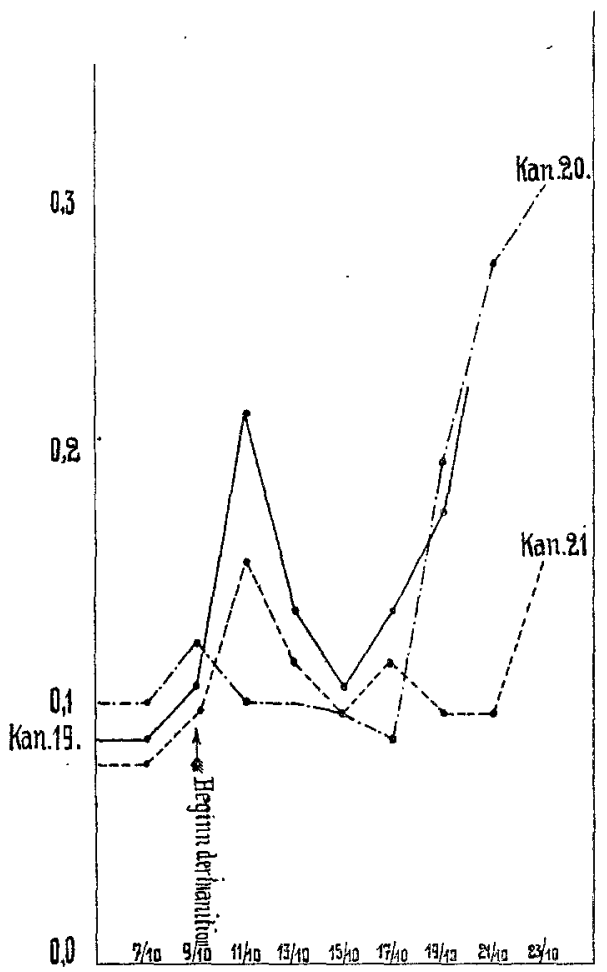

Kurve 17. (Serie IV.) P-Ausscheidung auf Kilogramm Körpergewicht berechnet. Kaninchen 19, 20 und 21.

der $10 \%$ ige Alkohol in einer geringeren Gabe auf die Lebensdauer, den Wasserbestand des Körpers (Urinmenge), die Erhaltung des Körpereiweisses (Ausscheirung von Stickstoff, Schwefel und Phosphor) einen günstigen Einfluss im Sinne einer Sparung ausznüben imstande ist. Grössere Gaben dagegen können schon toxisch wirken, indem sie die Lebensdauer des inaniierteu Kaninchens verkürzen, den Abbau des Eiweisses beschleunigen und den Flüssigkeitsbestand des Körpers schädigen. 
Aus den gewonnenen Analysenzahlen und den daraus zusammengestellten Tabellen und Kurven lassen sich noch einzelne interessante Tatsachen ableiten, die wir bisher noch nicht erwähnt haben.

Es ist uns nicht gelungen, in der uns zugänglichen Literatur Angaben darüber zu finden, dass nicht nur die Menge des ausgeschiedenen Stickstoffes, Phosphors und Schwefels, sondern auch die Urinmengen vor dem Tode eine „prämortale Steigerung" erfahren. Die prämortale Stickstoffsteigerung wird nach der Ansicht von

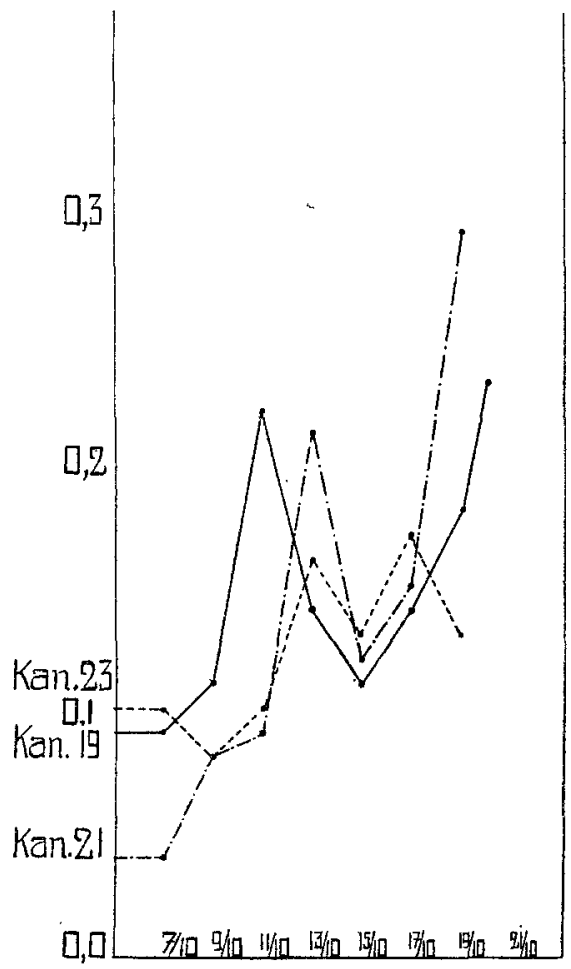

Kurve 18 (Serie IV.) P-Ausscheidung auf Kilogramm Körpergewicht berechnet. Kaninchen 19,22 und 23.

v. Voit ${ }^{1}$ ), welche neuerdings auch von Fr. N. Schulz ${ }^{2}$ ) anerkannt wird, auf eine grössere Inanspruchnahme des Körpereiweisses zurück-

1) C. v. Voit, Handb. d. Physiol. d. Gesamt-Stoffwechsels u. d. Fortpflanz. Leipzig 1881.

2) Fr. N. Schulz, E. Mangold, H. Stübel, E. Hempel, Beiträge zur Kenntnis des Stoffwechsels bei unzureichender Ernährung. Sep. Abdruck aus Arch. f. d. ges. Physiol. Bd. 114 S. 419. 1906. 
geführt, nachdem die Fettdepots mehr oder weniger aufgebraucht worden sind. Dass die „prämortale Harnflutsteigerung" mit diesen Umständen zusammenhängt, ist wohl zweifellos; dass sie aber nicht eine primäre, sondern eine sekundäre Erscheinung ist, braucht ebenfalls kaum erörtert zu werden. Wie man sich das in allen unseren Versuchen auftretende Phänomen zu erklären hat, darüber können nur Vermutungen angestellt werden. Man könnte sich einmal vorstellen, dass die Gewebe nicht mehr imstande sind, bei Fortschreiten des Zerfalles und Abbaues der Körpersubstanzen das Wasser mechanisch an sich zu halten. Dann aber wäre es möglich, dass beim vermehrten Abbau von Eiweiss mit durchschnittlich 25\% Sauerstoff während der prämortalen Stickstoffsteigerung eine grosse Menge Wasser neu gebildet und dann ausgesehieden wird, während vorher bei verhältnismässig geringem Eiweisszerfall auch nur wenig Wasser entstehen kann.

Auch eine Erklärung auf physikalisch-chemischer Grundlage erscheint niclit ausgeschlossen. Durch den Zerfall und Abbau der Eiweissstoffe gelangen auf einmal plötzlich die Eiweisstrümmer in die Blutbahn. Es entstehen auf diese Weise ans einem Eiweissmolekül eine mehr oder weniger grosse Anzahl von Molekülen, welche nicht mehr in den Geweben, sondern im Blut sich finden und dessen osmotischen Druck wesentlich erböhen müssen. Dadurch aber muss es notwendigerweise zu einer Wasserentziehung der Gewebe und Wasseranreicherung des Blutes kommen. Das vermehrte Blutwasser wird nun mit den stickstoff haltigen Abbauprodukten durch die Nieren ausgeschieden und gibt zu ler prämortalen Harnflutsteigerung Veranlassung.

Wir können natürlich vorläufig für keine der Anschauungen, welche wir als Erklärung gegeben haben, den Beweis erbringen, wir haben aber geglaubt, die Überlegungen, welche wir angestellt haben, anführen zu sollen.

Es lag ziemlich nahe, auch bei dieser Versuchsserie zu untersuchen, ob die Schwefel- und Phosphorausscheidung mit der Stickstoffausscheidung parallel ginge. Für gewöhnlich wird es ziemlich allgemein angenommen, nur vom Phosphor weiss man, dass er gegen Ende in relativ höherem Masse ausgesehieden wird als der Stickstoff. J: $\mathrm{Munk}^{\mathbf{1}}$ ) glaubt auf Grund seiner Versuche, dass diese Mehr-

1) J. Munk, Beiträge zur Stoffwechsel- und Ernährungslehre. Arch. f. d. ges. Physiol. Bd. 58 S. 309. 1894. 
ausfuhr von Phosphor darauf zurückzuführen sei, dass auch die Knochensubstanz wäbrend des Hungers abgebaut wird, und dass dabei phosphorsaure Salze frei werden. Seine Ansicht erfährt eine wesentliche Stütze in seinen Analysen des Kalk- und Magnesiumgehaltes des Urins bzw. des Kotes, durch die er zeigen konnte, dass diese beiden Substanzen ungefähr in demselben Verhältnis zum Phosphor, wie sie auch im Knochen vorhanden sind, eine Mehrausscheidung erfahren. Wir konnten bei unseren Kaninchen eine solche relative Vermehrung der Phosphorausscheidung nicht nachweisen. Bildet man nämlich den Quotienten aus $\mathrm{N}: \mathrm{P}$, so müsste dieser bei einer relativen Vermehrung der Phosphorausscheidung kleiner werden; gerade das Gegenteil aber war der Fall. Die Zahlen zeigen gegen das Ende der Inanition eine Tendenz zur Steigerung, wie aus der nachstehenden Tabelle XXIII zu ersehen ist.

Tabelle XXIII. Quotient N:P.

\begin{tabular}{|c|c|c|c|c|c|c|}
\hline $\begin{array}{c}\text { Datum } \\
1907\end{array}$ & $\begin{array}{c}\text { Kan. } \\
19\end{array}$ & $\begin{array}{c}\text { Kan. } \\
20\end{array}$ & $\begin{array}{c}\text { Kan. } \\
21\end{array}$ & $\begin{array}{c}\text { Kan. } \\
22\end{array}$ & $\begin{array}{c}\text { Kan. } \\
23\end{array}$ & Bemerkungen \\
\hline $\begin{aligned} \text { 7. } & \text { Okt. } \\
9 . & " \\
11 . & " \\
18 . & " \\
15 . & " \\
17 . & " \\
19 . & " \\
21 . & " \\
23 . & "\end{aligned}$ & $\begin{array}{r}7,07 \\
3,00 \\
3,73 \\
7,07 \\
8,02 \\
6,98 \\
12,78 \\
8,45\end{array}$ & $\begin{array}{l}2,70 \\
3,31 \\
4,71 \\
5,98 \\
8,74 \\
9,47 \\
8,72 \\
7,82 \\
8,63\end{array}$ & $\begin{array}{r}6,72 \\
4,29 \\
4,14 \\
7,01 \\
8,30 \\
7,46 \\
9,31 \\
11,39 \\
10,00\end{array}$ & $\begin{array}{r}10,98 \\
5,40 \\
4,00 \\
4,78 \\
7,70 \\
9,02 \\
9,20\end{array}$ & $\begin{array}{l}4,81 \\
4,03 \\
2,35 \\
6,54 \\
6,67 \\
8,36 \\
9,83\end{array}$ & $\begin{array}{l}\text { Kaninchen } 22 \text { und } 23 \text { gestoiben } \\
20 . \text { Olkt. } 1907 \text { Kaninchen } 19 \text { gest. } \\
\text { Kaninchen } 20 \text { und } 21 \text { gestorben. }\end{array}$ \\
\hline
\end{tabular}

Es dürfte aber kaum möglich sein, diese Zahlen gegen die allgemein angenommene Ansicht von I. Munk zu verwerten; denn man könnte bei den Versuchen an Kaninchen mit einer gewissen Berechtigung den Einwand erheben, die niedrigen Werte für den Quotienten N:P, also die relativ hohen Phosphorwerte kämen im Anfang der Inanition dadurch zustande, dass noch phosphorsaure Salze aus dem Intestinaltraktus resorbiert würden, was dann im weiteren Verlauf des Versuches und besonders gegen Ende desselben nicht mehr der Fall sein dürfte. Auf diese Weise liess sich dann ungezwungen die Steigerung des Quotienten N:P, die relativ geringe Phosphorausfuhr im zweiten Teil des Versuches erklären. Wenn während der vollen Ernährung für den Quotienten N:P hohe Werte gefunden werden, so liegt dies wohl daran, dass beim Pflanzen- 
fresser wenigstens ein Teil der Phosphorsäure durch den Kot ausgeschieden wird. Bergmann ${ }^{1}$ ) zeigte für den Hammel, dass bei hinreichender Nahrung nur Spuren von Phosphorsäure im Harn nachweisbar sind und diese sonst durch den Kot ausgesehieden werden. Wenn die Verallgemeinerung, dass dies Verhalten für alle Pflanzenfresser zutreffe, auch nicht richtig ist, so ist doch andererseits nicht in Abrede zu stellen, dass wenigstens ein Teil der resorbierten Phosphorsäure beim Pflanzenfresser den Organismus mit den Fäzes verlässt. Wenn diese Ansicht zu Recht besteht, so würden aus dem Quotienten N:P bei Kaninchen Schlussfolgerungen kaum zu ziehen sein, und wir können schon aus diesem Grunde auf weitere Ausführungen verzichten.

Tabelle XXIV. Quotient N:S.

\begin{tabular}{|c|c|c|c|c|c|c|}
\hline $\begin{array}{c}\text { Datum } \\
1907\end{array}$ & $\begin{array}{c}\text { Kan. } \\
19\end{array}$ & $\begin{array}{c}\text { Kan. } \\
20\end{array}$ & $\begin{array}{c}\text { Kan. } \\
21\end{array}$ & $\begin{array}{c}\text { Kan. } \\
22\end{array}$ & $\begin{array}{c}\text { Kan. } \\
23\end{array}$ & Bemerkungen \\
\hline $\begin{aligned} \text { 7. } & \text { Okt. } \\
9 . & " \\
11 . & " \\
13 . & " \\
15 . & " \\
17 . & " \\
19 . & " \\
20 . & " \\
21 . & " \\
23 . & "\end{aligned}$ & $\begin{array}{r}4,09 \\
2,7 \\
7,2 \\
20,9 \\
22,4 \\
12,7 \\
9,9 \\
10,2\end{array}$ & $\begin{array}{r}3,0 \\
4,0 \\
13,7 \\
16,5 \\
14,0 \\
14,2 \\
11,0 \\
\\
9,3 \\
10,9\end{array}$ & $\begin{array}{c}4,86 \\
3,7 \\
3,5 \\
18,7 \\
20,6 \\
7,5 \\
13,5\end{array}$ & $\begin{array}{l}2,57 \\
3,3 \\
13,6 \\
18,8 \\
13,1 \\
10,2 \\
11,3\end{array}$ & $\begin{array}{c}3,56 \\
3,1 \\
7,4 \\
15,2 \\
6,7 \\
12,0 \\
12,1\end{array}$ & $\begin{array}{l}\text { Kaninchen } 22 \text { und } 23 \text { gestorben } \\
20 \text {. Okt. } 1908 \text { Kaninchen } 19 \text { ge- } \\
\text { storben } \\
\text { Kaninchen } 20 \text { und } 21 \text { gestorben }\end{array}$ \\
\hline
\end{tabular}

Auf Tabelle XXIV haben wir es noch unternommen, in analoger Weise die Quotienten N:S auszurechnen. Wir können aus den Zahlen die schon früher konstatierte Tatsache entnehmen, dass mit Beginn der Inanition der Quotient $\mathrm{N}: \mathrm{S}$ stark in die Höhe geht. Dies ist wohl darauf zurückzuführen, dass beim Fehlen der Nahrung. die Fäulnis im Darm vermindert wird und wenig oder gar keine Gallensäuren in den Darm gelangen, Umstände, welche normalerweise als eine der Quellen der Harnschwefelsäure zu gelten haben. Beim Fortschreiten des Versuches werden die Zahlen kleiner, d. h. im weiteren Verlauf nimmt. relativ zur Stickstoffausscheidung die Schwefelausscheidung im Urin zu. Da von einer Resorption von

1) W. Bergmann, Über die Ausscheidung der Phosphorsäure beim Fleischund Pflanzenfresser. Arch. f. exper. Path. u. Pharm. Bd. 47 S. 77. 1902. 
Schwefelverbindungen aus dem Darm beim Fortsetzen der Inanition keine Rede sein kann, so muss man schon annehmen, dass im Körper gegen Ende derselben Gruppen von Eiweisssubstanzen zerstört werden, welche reich an Schwefel sind.

Ein besonders in die Augen fallender Unterschied zwischen den einzelnen Tieren der Versuchsreihe bezüglich des Verhaltens des Quotienten $\mathrm{N}: \mathrm{S}$ ist nicht zu konstatieren. Nur bei der Berechnung des Quotienten $\mathrm{N}: \mathrm{S}$ aus der Gesamtmenge des während der Inanition ausgeschiedenen Stickstoffes und Schwefels sind einige Differenzen zu bemerken. $\mathrm{N}: \mathrm{S}$ bei Kaninchen Nr. 19 beträgt 11,57, bei Kaninchen Nr. 20 und Nr. 21 11,77 bzw. 9,83, bei Tier Nr. 22 und Nr. 23 12,43 bzw. 12,59. Während also die „3-cem-Alkoholtiere" einen gleichen oder niedrigeren Quotienten aufweisen, ist bei den „5-ccm-Alkoholtieren" der Quotient höher geworden. Es ist also bei diesen Tieren wohl eine relativ schwefelarme Eiweisssubstanz in höherem Grade zerstört als bei den anderen Kaninchen und dies auf Kosten eines anderen Eiweisskörpers. $\mathrm{Ob}$ dies die Nucleine sind, wie Rosenfeld ${ }^{1}$ ) und sein Schüler Pringshei ${ }^{2}$ ) beim Menschen auf Grund ihrer Harnsäurezahlen annehmen, muss vorderhand dahingestellt bleiben.

So einwandfrei die Versuchsreihe 4 eine günstige Wirkung des Alkohols in kleinen Gaben und, was a priori anzunehmen war, eine schädliche bei Verabreichung grösserer Gaben erwiesen hatte, so glaubten wir doch noch eine fünfte Versuchsserie anstellen zu sollen um die Zahl und damit die Beweiskraft unserer Versuche zu erhöhen.

\section{Versuchsreihe.}

$\mathrm{Zu}$ diesem Versuche wurden vier Kaninchen eingestellt. Die experimentellen Bedingungen waren genau die gleichen wie bei Serie IV, nur dass die Alkoholgaben bzw. die Mengen des subkutan beigebrachten Wassers während der Inanition auf Körpergewicht berechnet wurden, so dass die Tiere beim Fallen des Körpergewichtes auch proportional kleinere Gaben von Alkohol und Wasser erhielten. Zwei der Tiere dienten als Kontrolltiere und erhielten Wasser, die anderen beiden bekamen Alkohol zu 10 .Volumprozent in fallenden Dosen.

1) G. Rosenfeld, l. c.

2) Pringsheim, l. c. 
Serie $V$.

Tabelle XXV. Kaninchen 24 .

\begin{tabular}{|c|c|c|c|c|}
\hline $\begin{array}{l}\text { Datum } \\
1908\end{array}$ & $\begin{array}{c}\text { Körper- } \\
\text { gewicht } \\
\mathrm{g}^{*}\end{array}$ & $\begin{array}{l}\text { Gewichtsverlust } \\
\text { in } \mathrm{g} \text { und } \%\end{array}$ & $\begin{array}{c}\text { Urinmenge } \\
\text { cem }\end{array}$ & Bemerkungen \\
\hline \multirow[t]{2}{*}{ 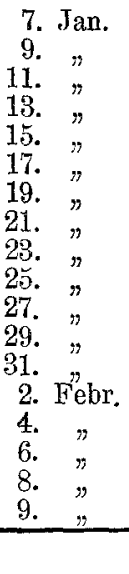 } & $\begin{array}{l}2377 \\
2480 \\
2498 \\
2405 \\
2422 \\
2222 \\
2117 \\
2028 \\
1925 \\
1845 \\
1777 \\
1705 \\
1640 \\
1575 \\
1515 \\
1443 \\
1367 \\
1310 \\
\end{array}$ & 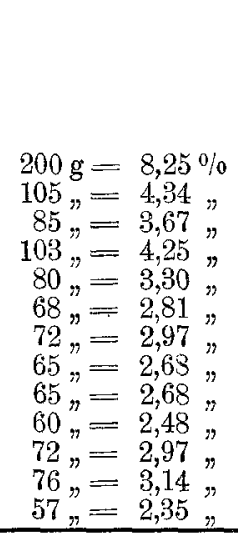 & $\begin{array}{r}250 \\
175 \\
325 \\
270 \\
280 \\
145 \\
55 \\
55 \\
65 \\
50 \\
40 \\
45 \\
45 \\
40 \\
40 \\
50 \\
55 \\
40 \\
\end{array}$ & $\begin{array}{l}\text { Beginn der Inanition } \\
\text { - } \\
\text { Gestorben }\end{array}$ \\
\hline & - & $1112 \mathrm{~g}=45,91 \%$ & 725 & \\
\hline
\end{tabular}

Tabelle XXVI. Kaninchen 25.

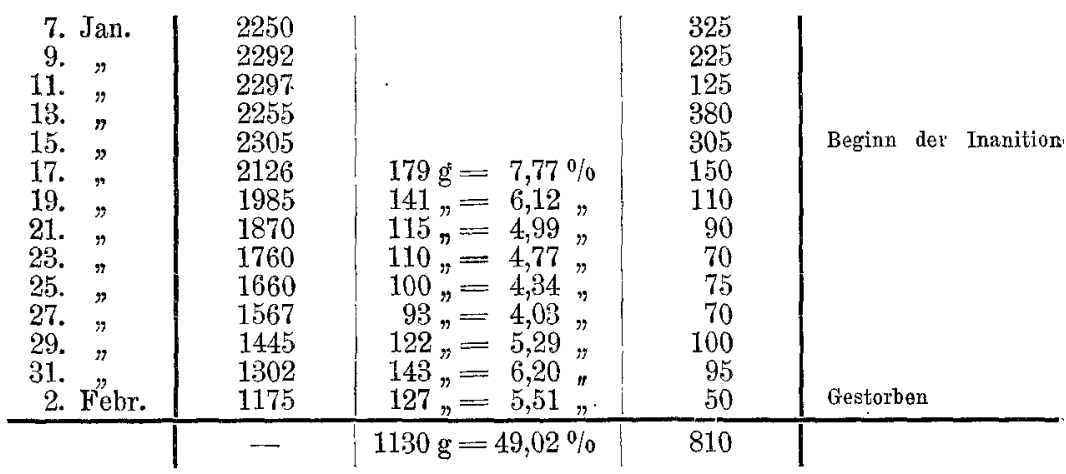

Tabelle XXVII. Kaninchen 26.

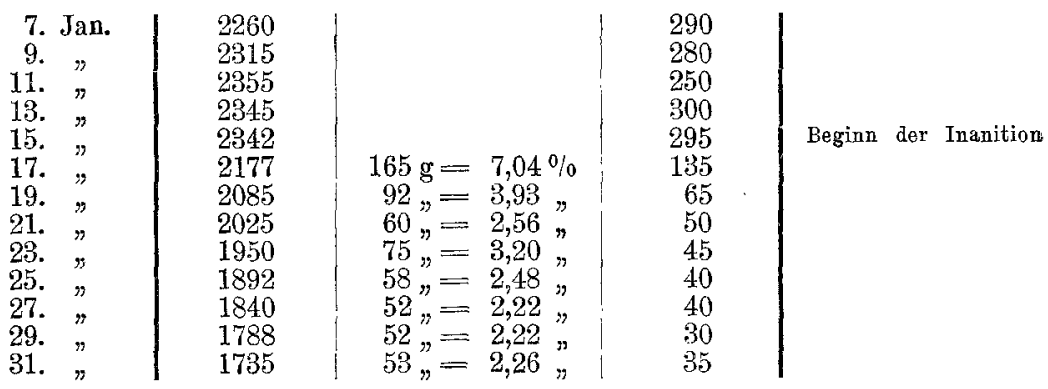


Tabelle XXVII. (Fortsetzung.)

\begin{tabular}{|c|c|c|c|c|}
\hline $\begin{array}{c}\text { Datum } \\
1908\end{array}$ & $\begin{array}{l}\text { Körper- } \\
\text { gewicht g }\end{array}$ & $\begin{array}{l}\text { Gewichtsverlust } \\
\text { in } g \text { und } \%\end{array}$ & $\begin{array}{l}\text { Urinmenge } \\
\text { ccm }\end{array}$ & Bemerkungen \\
\hline 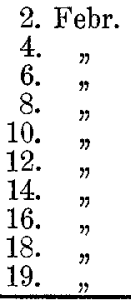 & $\begin{array}{l}1683 \\
1630 \\
1572 \\
1520 \\
1462 \\
1410 \\
1355 \\
1295 \\
1197 \\
1105 \\
\end{array}$ & $\begin{array}{l}52 \mathrm{~g}=2,22 \% \\
53 "=2,26 " \\
58 "=2,48 " \\
52 "=2,22 " \\
58 "=2,48 " \\
52 "=2,22 " \\
55 "=2,35 " \\
60 "=2,56 " \\
98 "=4,18 " \\
92 "=3,93 "\end{array}$ & $\begin{array}{l}30 \\
35 \\
30 \\
35 \\
35 \\
30 \\
35 \\
40 \\
65 \\
55 \\
\end{array}$ & Gestorben \\
\hline & & $1237 \mathrm{~g}=52,82 \%$ & $825 \mathrm{ccm}$ & \\
\hline
\end{tabular}

Tabelle XXVIII. Kaninchen 27.

\begin{tabular}{|c|c|c|c|c|}
\hline 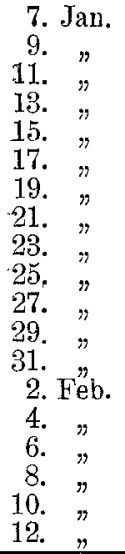 & $\begin{array}{l}2285 \\
2830 \\
2360 \\
2310 \\
2325 \\
2145 \\
2008 \\
1900 \\
1820 \\
1762 \\
1697 \\
1632 \\
1560 \\
1510 \\
1452 \\
1385 \\
1314 \\
1243 \\
1215\end{array}$ & 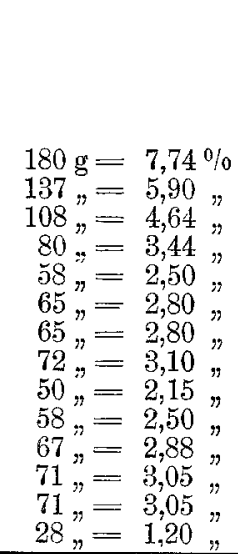 & $\begin{array}{l}310 \\
255 \\
250 \\
350 \\
280 \\
125 \\
100 \\
70 \\
55 \\
40 \\
50 \\
40 \\
50 \\
35 \\
40 \\
40 \\
45 \\
30 \\
20\end{array}$ & Beginn der Inanition \\
\hline & & $1110 \mathrm{~g}=47,74 \%$ & 740 & \\
\hline
\end{tabular}

Die Tiere wurden vor Beginn des Versuches 2 Monate lang bei gleichem Futter gehalten und ihr Körpergewicht sowie ihr sonstiges Verhalten genau beobachtet. Auf den Tabellen XXV bis XXVIII, aus denen die Einzelheiten zu ersehen sind, führen wir nur die letzten Tage der Fütterung an.

Die Tabellen zeigen uns also, dass die mit fallenden Alkoholgaben behandelten Tiere länger lebten als die Kaninchen, denen nur analoge Mengen von destilliertem Wasser subkutan beigebracht worden waren. Das Anfangsgewicht der Tiere betrug von Kaninchen Nr. $24.2422 \mathrm{~g}$ und von Nr. $252305 \mathrm{~g}$; beide Tiere erhielten Wasser. Die beiden Alkoholtiere Nr. 26 und Nr. 27 besassen ein Anfangs- 
gewicht von $2342 \mathrm{~g}$ bzw. $2145 \mathrm{~g}$. Die "Normalhungertiere" lebten 26 bzw. 19 Tage, während die "Alkoholhungertiere" 36 bzw. 29 Tage die Inanition ertrugen, also länger lebten. Dabei war das Körpergewicht der Alkobolhungertiere kleiner als das der Kontrolltiere; denn Kaninchen Nr. 24 war $18 \mathrm{~g}$ schwerer als Tier Nr. 26, und Kaninchen Nr. 26 übertraf Tier Nr. 27 um 160 g. Die Kaninchen Nr. 25 und 26 waren ungefähr gleich, und trotzdem lebte das Alkoholhungertier wesentlich länger.

Auch bei Betrachtung der Körpergewichte kann man sich dem Gedanken nicht verschliessen, dass der Alkohol sehr günstige Wirkungen gezeigt habe. Tier Nr. 24 hat 45,91\% seines Anfangsgewichtes und Kaninchen Nr. 25 sogar $49,02 \%$ eingebüsst. Im gleichen Zeitraum dagegen haben die beiden Alkoholtiere $36,34 \%$ bzw. $42,95 \%$ verloren, selbst wenn die Überlebensdauer des Kaninehens Nr. 24 der Rechnung zugrunde gelegt wird.

Die Diurese war bei den Alkoholhungertieren geringer als bei den Kontrolltieren. Kaninchen Nr. 24 und Nr. 25 schieden bis zum Tode $725 \mathrm{ccm}$ bzw. $810 \mathrm{ccm}$ Urin aus, die beiden Alkoholhungertiere nur $583 \mathrm{ccm}$ und $705 \mathrm{ccm}$.

So sehen wir denn, dass auch bei dieser Versuchsreihe der Alkohol in geringen Gaben einen günstigen Einfluss auf die Lebens. dauer hungernder Tiere ausgeübt hat. (Siehe Kurven Nr. 19-21.)

Tabelle XXIX.

\begin{tabular}{|c|c|c|c|c|c|}
\hline $\begin{array}{l}\text { Kan. } \\
\text { Nr. }\end{array}$ & $\begin{array}{l}\text { Anfangs- } \\
\text { gewicht } \\
\text { g }\end{array}$ & Gewichtsverlust & $\begin{array}{l}\text { Urin- } \\
\text { mengen } \\
\mathrm{g}\end{array}$ & $\begin{array}{l}\text { Lebens- } \\
\text { dauer } \\
\text { Tage }\end{array}$ & Bemerkungen \\
\hline $\begin{array}{l}24 \\
25 \\
26\end{array}$ & $\begin{array}{l}2422 \\
2305 \\
2342\end{array}$ & $\begin{array}{l}1112 \mathrm{~g}=45,91 \% \\
1130 \%=49,02 \% \\
1287 "=52,82 \%\end{array}$ & $\begin{array}{l}725 \\
810 \\
825(583)\end{array}$ & $\begin{array}{l}26 \\
19 \\
36\end{array}$ & $\begin{array}{l}\text { Die eingeklammerten } \\
\text { Zahlen bedenten den } \\
\text { Gewichtsverlust bzw. } \\
\text { die UrinmengeIt }\end{array}$ \\
\hline 27 & 2325 & $1110 "=\begin{array}{r}(30,74,74 \\
(42,95 ")\end{array}$ & $740(705)$ & 29 & $\begin{array}{l}26 \text { Tage d. Inanition, } \\
\text { der Lebensaner des } \\
\text { Kontrolltieres Nr. } 24 .\end{array}$ \\
\hline
\end{tabular}

Wenn es erlaubt ist, die Gesamtlebensdauer der Kaninchen, welchen Alkohol verabreicht wurde, mit den Kontrolltieren zu vergleichen, so lässt sich folgende Berechnung anstellen, welcher die früher gepflogenen Überlegungen bezüglich der Möglichkeit des Vergleiches der einzelnen Tiere zugrunde gelegt wurden. Sechs Kontrolltiere mit einem Gesamtkörpergewicht von $13047 \mathrm{~g}$ lebten zusammen 105 Tage, die sechs Alkoholtiere, denen die kleineren Alkoholgaben 
gereicht wurden, wogen $12522 \mathrm{~g}$ und lebten 128 Tage. Mit anderen Worten: Von den Kontrolltieren wog jedes Kaninchen durchschnittlich $2174,5 \mathrm{~g}$ und lebte $171 / 2$ Tage, und von den Tieren, welche die kleineren Alkoholgaben empfangen hatten, betrug trotz des geringeren durchschnittlichen Gewichtes eines jeden Tieres von $2087 \mathrm{~g}$ die Lebensdauer $21 \%$ Tage; jedes Kaninchen lebte also nahezu 4 Tage länger als die Kontrolltiere. Die Kaninchen der drei letzten Versuchsreihen, denen die grösseren Alkoholgaben beigebracht waren, wogen

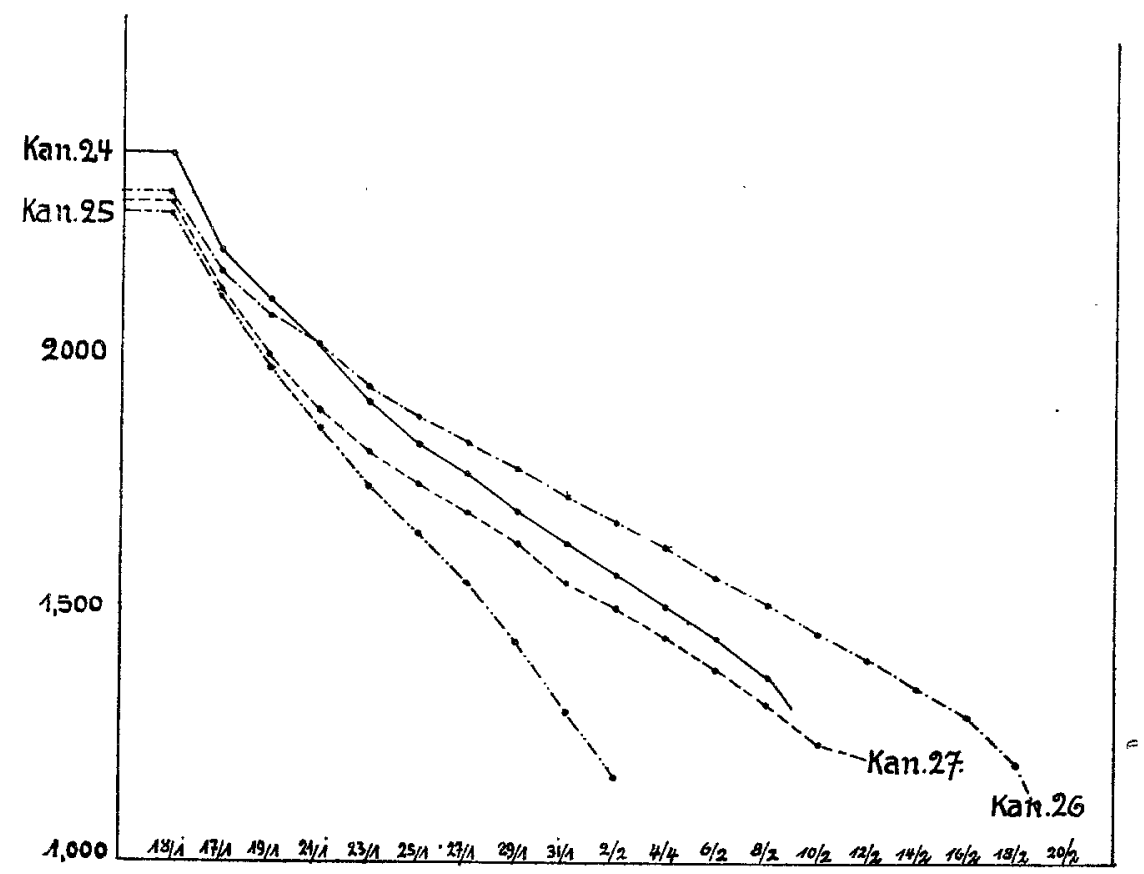

Kurre 19. (Serie V.) Körpergewicht in Gramm. Kaninchen 24, 25, 26 und 27.

im Durchschnitt 1970,3 g und lebten 11 Tage. Ihre Lebensdauer ist um $6^{1 / 2}$ Tage kleiner als die der Kontrolltiere.

Es lässt sich daher nicht leugnen, dass kleinere Gaben von Alkohol bei hungernden Tieren die Lebensdauer verlängern können; aber ebenso unbestritten ist es, dass grössere Gaben des Alkohols den Tod hungernder Tiere beschleunigen. Die Antwort nach dem Grund und der Ursache dieser Tatsachen geben uns teilweise die Stoffwechselversuche der vierten Serie. Es zeigt sich, dass der Alkohol ebenso wie beim normal ernährten Menschen und Tiere 
auch beim hungernden Organismus die Eiweisssubstanzen vor der Verbrennung, den Körper vor Wasserverlusten durch den Urin längere Zeit bewahrt, so dass auch das Körpergewicht bei den mit den kleinen Alkoholgaben behandelten Tieren langsamer abfällt als bei den Kontrolltieren.

Wenn diese Schonung des Eiweisses und des Wasserbestandes im Organismus die einzige Ursache für die Verlängerung der Lebensdauer der Kaninchen wäre, denen die kleineren Alkoholgaben dargereicht wurden - bei dem Unsatz des Körperfettes wird der Alkohol wohl ähnliche Wirkungen ausüben wie beim normalen Menschen -, so müssten diese Tiere folgerichtig dann sterben,

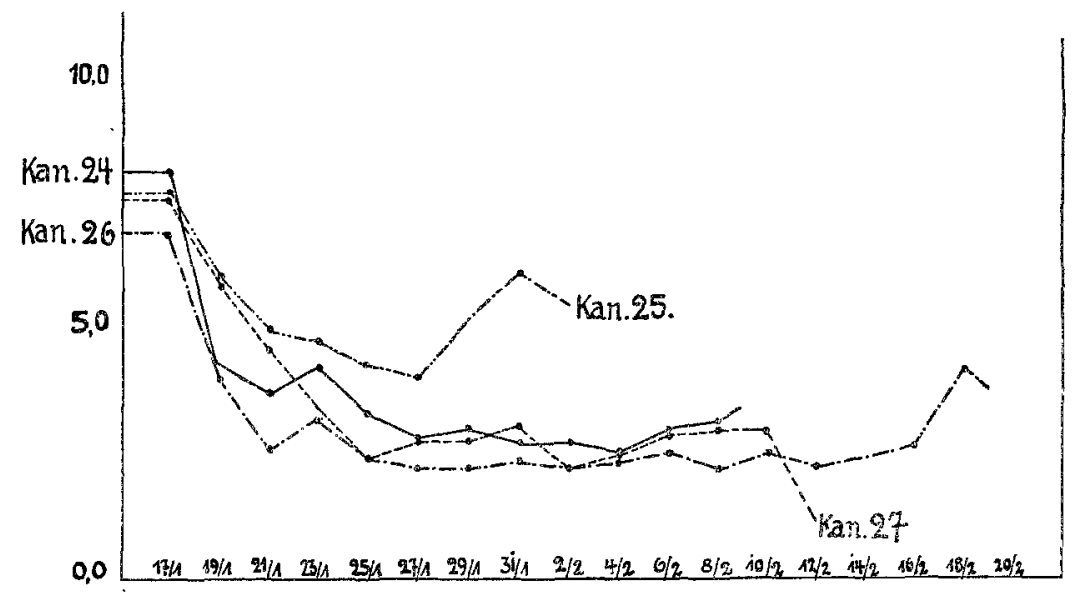

Kurve 20. (Serie V.) Körpergewichtsverlust in Prozenten. Kaninchen 24, 25, 26 und 27.

wenn ihr Eiweiss- und Wasserverlust dem der Kontrolltiere gleichkommt. Aus den Tabellen aber lässt sich mit Leichtigkeit die interessante Tatsache entnehmen, dass der Gesamtverlust an Eiweiss, Wasser und Körpergewicht der Alkoholtiere beim Tode grösser ist als bei den Kontrolltieren.

Wie müssen wir uns dann die längere Lebensdauer erklären? Verschiedene Möglichkeiten konnten dafür in Frage kommen. Zu* nächst hatten wir die Vermutung, dass vielleicht unter dem Einfluss des Alkohols der Eiweissbestand der lebenswichtigsten Organe besonders geschont würde und andere auf deren Kosten Eiweiss verlören. Um zu prüfen, $o b$ diese Vermutung berechtigt wäre, unterwarfen wir einige Organe, nämlich die Knochen, Muskeln, die Leber 
und das Herz einer analytischen Untersuchung, indem wir in folgender Weise vorgingen: Die genannten Organe und Gewebe wurden dem eben gestorbenen Tiere entnommen und auf dem Wasserbade vorgetrocknet, dann im Porzellanmörser zerkleinert und nach weiterem

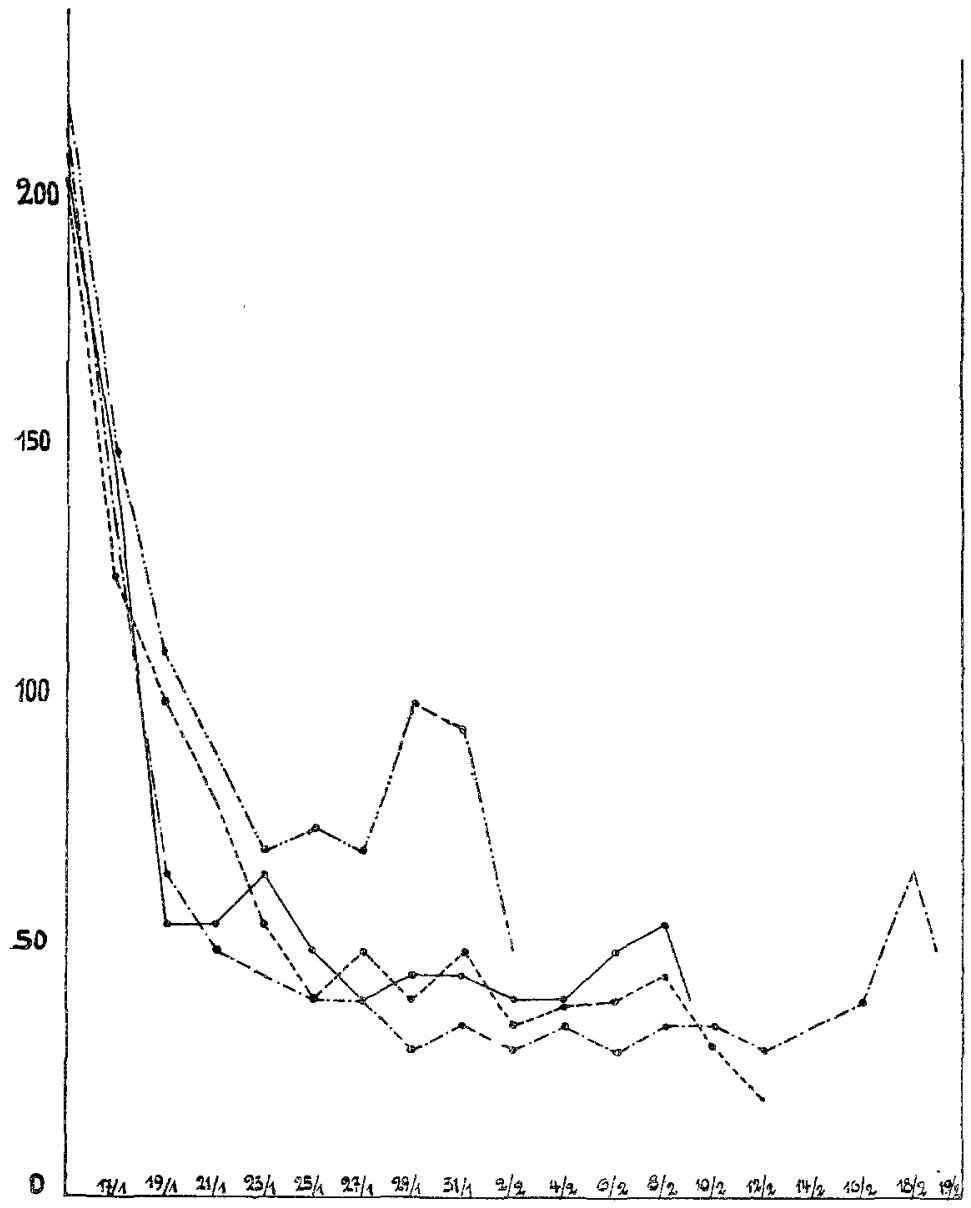

Kurve 21. (Serie V). Urinmengen der Kaninchen 24, 25, 26 und 27.

Trocknen pulverisiert. Dies so gewonnene Organ- bzw. Gewebspulver wurde nun bei $105-108^{\circ}$ in Trockenofen bis zur Konstanz getrocknet. Ein aliquoter Teil wurde verascht und in der Asche Eisen, Calcium, Magnesium und Phosphor bestimmt. Die Ergebnisse dieser Analysen werden von dem einen von uns an anderer Stelle veröffentlicht werden; dabei wird sich auch Gelegenheit 
bieten, die angewandten analytischen Methoden zu besprechen. Die gewonnenen Zahlen sind der Vollständigkeit halber in der weiter unten stehenden Tabelle XXX angeführt. Die aus der Trockensubstanz erbaltene Asche wurde gewogen und auf 100 Teile der Trockensubstanz verrechnet. In einem anderen Tejle der Trockensubstanz wurde der Stickstoff mit Hilfe der Kjeldahl-Methode bestimmt und auf Eiweiss berechnet, indem die Stickstoffzahl mit 6,25 multipliziert wurde. Wir kannten nunmehr in den Geweben den prozentischen Aschegehalt und die prozentuelle Stickstoffbzw. Eiweissmenge. Der Rest musste aus Kohlehydraten und Fett bestehen.

Serie III.

Tabelle XXX.

Analysen der Knochen.

\begin{tabular}{l|c|c|c|c|c|c|c|c}
\hline & $\mathrm{Fe}$ & $\mathrm{Ca}$ & $\mathrm{Mg}$ & $\mathrm{P}$ & Asche & $\mathrm{N}$ & Eiweiss & $\begin{array}{r}\text { Kohle- } \\
\text { hydrate } \\
\text { u. Fett }\end{array}$ \\
\hline $\begin{array}{l}\text { Normale Tiere . } \\
\text { Normale Hungertiere } \\
\text { Alkoholhungertiere. }\end{array}$ & 0,0455 & 21,4634 & 0,2059 & 10,1970 & 56,8216 & 4,2079 & 26,2994 & 16,8790 \\
25,5806 & 0,2273 & 13,8934 & 67,4117 & 4,2957 & 26,8413 & 5,7470 \\
9,7807
\end{tabular}

Analysen des Muskels.

\begin{tabular}{l|l|l|l|l|l|l|l|r|} 
Normale Tiere . & 0,0159 & 0,1306 & 0,1370 & 1,0900 & $\mathbf{5 , 2 0 1 9}$ & $\mathbf{1 4 , 0 2 9 6}$ & 87,6850 & 7,1131 \\
Normale Hungertiere & 0,0121 & 0,2419 & 0,1054 & 1,0699 & 5,2842 & 11,8752 & 74,2200 & $19 ; 4958$ \\
Alkoholhungertiere. & 0,0206 & 0,1030 & 0,0541 & 0,9064 & 4,8051 & 10,7444 & 67,1525 & 28,0424
\end{tabular}

Analysen der Leber.

\begin{tabular}{l|l|l|l|l|l|r|r|r} 
Normale Tiere . & 0,0572 & 0,0234 & 0,0575 & 1,0401 & 4,2023 & 8,8997 & 55,6231 & 40,1746 \\
Normale Hungertiere & 0,7530 & 0,0549 & 0,0046 & 1,3305 & 5,7661 & 11,7560 & 73,4750 & 20,7589 \\
Alkoholhungertiere . & 0,2302 & 0,0921 & 0,0783 & 1,1111 & 5,4620 & 11,7048 & 73,1550 & 21,3830
\end{tabular}

Analysen der Herzen.

\begin{tabular}{|c|c|c|c|c|c|c|c|c|}
\hline Normale Tiere : . & 0,19 & 0,0672 & 0,0507 & 0,6961 & $\rightarrow$ & - & 7 & \\
\hline eHungertiere & 0,0070 & 0,0702 & 0,1405 & 1,0747 & 5,5212 & 11,7451 & 73,4069 & 21,071 \\
\hline A. & & & & 0,8415 & 5,9994 & 11,4825 & 71,7656 & \\
\hline
\end{tabular}

Die so erhaltenen Zablen geben uns Aufschluss darüber; in welcher Weise die einzelnen Gewebe, wenn es zu sagen erlaubt ist, ihre Konstitution verändert haben. Alle Gewebe nehmen ja im Hunger absolut ab, und wenn wir, wie beim Muskel, eine Zunahme z. B. an Kohlehydraten und Fetten sehen, so handelt es sich nur um eine relative Zunahme, indem andere Substanzen wie das Eiweiss eine verhältnismässig stärkere Einbusse erlitten haben. Wir - werden infolgedessen aus einer relativen Zunahme nichts 
schliessen können; dagegen wird es möglich sein, aus einem starken Defizit eines Bestandteiles zu entnehmen, dass die Gewebe besonders an diesen Komponenten ihrer Zusammensetzung eine Einbusse erlitten haben. Im besonderen werden wir imstande sein, nachzuweisen, ob unter dem Einflusse des Alkohols ein bestimmtes Gewebe mehr Eiweiss verloren hat als beim einfachen Hungern. So sehen wir denn, dass beim Herzen - gewiss einem der lebenswichtigsten Organe - ebenso wie in der Leber der Eiweissgehalt der Alkoholbungertiere nur unwesentlich gegenüber den Kontrolltieren vermindert ist; dagegen ist die Eiweisskomponente im Knochen, in welchem sie bei den Normalhungertieren gar nicht angegriffen wird, und im Muskel bei den Alkoholhungertieren viel stärker in Mitleidenschaft gezogen.

Man könnte also den Schluss ziehen, dass unter dem Einfluss des Alkohols bei hungernden Tieren der Eiweissbestand der lebenswichtigen Organe geschont wird, und dies auf Kosten von Geweben, deren Zerfall das Leben weniger bedroht. Unsere Annahme würde somit durch diese Untersuchungen eine Stütze erfahren haben.

Wir geben aber zu, dass man sich die Verhältnisse noch anders vorstellen kann. Die von $\mathrm{Binz}^{1}$ ) und seinen Schülern bewiesene stimulierende Wirkung des Alkohols auf die Atmung, die von einer Reihe von Autoren gezeigte günstige Beeinflussung des Kreislaufes können es bewirken, dass die Organe trotz eines Eiweissverlustes, welcher den der Normalhungertiere übersteigt, noch funktionieren, wo letztere nicht mehr den Dienst leisten können.

Noch andere Umstände mögen ihre Hand bei der günstigen Wirkung des Alkobols auf die Lebensdauer im Spiele haben. Nach Tigerstedtª ${ }^{2}$ z. B. ist die Möglichkeit nicht auszuschliessen, dass in der Inanition besonders kurz vor dem Tode giftige Stoffwechselprodukte entstehen, welche nach diesem Autor z. B. die prämortale Stickstoffsteigerung im Urin mitbedingen. Vom Alkohol wissen wir aber, dass er eine ganze Reihe von Substanzen entgiften kann. Wir wollen da nur an die Arbeiten Reid-Hunt's ${ }^{3}$ ) erinnern, welcher

1) C. Binz, Über den Alkohol als Arzneimittel. Vortrag in der Hufeland'schen Gesellschaft. 1902.

2) R. Tigerstedt, Die Physiologie des Stoffwechsels in W. Nagel's Handb. d. Physiol. des Menschen Bd. 1. Braunschweig 1906.

3) Reid-Hunt, Zur Kenntnis der Toxikologie einiger Nitrile und deren Antidote. Arch. int. de Pharmacodyramie et de Thérapie t. 12 p. 447.1904. 
zeigen konnte, dass der Alkohol ein wirksames Gegengift gegen gewisse Nitrile ist. In ähnlicher Weise könnte der Alkohol auch hier antitoxisch gewirkt haben.

\section{Zusammenfassung.}

Am Schluss unserer experimentellen Untersuchungen sei es uns gestattet, die Versuchsergebnisse zusammenzufassen und in kurzen Sätzen einige Folgerungen wiederzugeben, welche aus den Versuchen gezogen werden können.

1. In passender Dosierung ist es durch subkutan beigebrachten Alkohol möglich, die Lebensdauer hungernder Kaninchen zu verlängern.

2. Grössere Alkoholgaben beschleunigen den Tod der Versuchstiere.

3. Die günstige Einwirkung des Alkohols ist sicher zum Teil seiner eiweisssparenden Wirkung und der besseren Erhaltung des Wasserbestandes des Organismus zuzuschreiben.

4.. Ein anderer Teil der günstigen Wirkung des Alkohols dürfte darauf beruhen, dass unter seinem Einfluss der Eiweissbestand lebenswichtiger Organe auf Kosten auderer, für das Fortbestehen des Lebens minder wertvoller Gewebe erhalten bleibt.

5. Die Beschleunigung des Eintrittes des Todes unter dem Einfluss grösserer Alkoholgaben lässt sich ungezwungen durch vermehrten Eiweisszerfall erklären, der, wie der Quotient N : S zeigt, besonders die schwefelärmeren Eiweisssubstanzen ergreift.

6. Die Stoffwechselwirkungen des Alkobols bei hungernden Kaninchen finden auch im Verhalten des Körpergewichts ihren Ausdruck.

7. Eine diuretische Wirkung des Alkohols tritt nur bei Verabreichung höherer Gaben auf, kleinere schränken die Diurese ein.

8. Wenn es erlaubt ist, die an hungernden Tieren gewonnenen Resultate auf den Menschen zu übertragen, so kommt dem Alkohol in passender Dosis, welche toxische Wirkungen ausschliesst, in vielfacher Beziehung eine hohe Bedeutung zu. Sowohl bei der Behandlung unzureichend ernährter Patienten (Fiebernde und solche, welche die Nahrungsaufnahme verweigern, oder bei. denen sie aus einem anderen Grunde erschwert ist) als auch vom militärischen und sportlichen 
Gesichtspunkte aus kann der Alkohol eine grosse Rolle spielen, da er imstande ist, das Leben hungernder Organismen zu verlängern und Fälle von Unmöglichkeit der Ernährung sowohl im Felde als auch bei Ausübung des Sportes auftreten können.

\section{Analytische Belege.}

\section{Serie III.}

Schwefelbestimmungen.

Urin der Kaninchen Nr. 13 und 14.

9. Mai 1907. a) $\left.0,0309 \mathrm{~g} \mathrm{BaSO}_{4}\right\}=0,004175 \mathrm{~g} \mathrm{~S}$. In $610 \mathrm{ccm}$ Urin $+140 \mathrm{ccm}$ b) $\left.0,0315 \mathrm{~g} \mathrm{BaSO}_{4}\right\} \quad$ Spülwasser $0,3131 \mathrm{~g} \mathrm{~S}$.

10. Mai 1907. a) $0,03093 \mathrm{~g} \mathrm{BaSO}_{4} \mid=0,0043 \mathrm{~g} \mathrm{~S}$. In $750 \mathrm{ccm}$ Urin $+65 \mathrm{ccm}$ b) $\left.0,03183 \mathrm{~g} \mathrm{BaSO}_{4}\right\} \quad$ Spülwasser $0,3504 \mathrm{~g} \mathrm{~S}$.

12. Mai 1907. a) $\left.0,04323 \mathrm{~g} \mathrm{BaSO}_{4}\right\}=0,0059 \mathrm{~g} \mathrm{~S}$. In $250 \mathrm{ccm}$ Urin $+65 \mathrm{ccm}$ b) $\left.0,04333 \mathrm{~g} \mathrm{BaSO}_{4}\right\} \quad$ Spülwasser $0,1859 \mathrm{~g} \mathrm{~S}$.

14. Mai 1907. a) $0,02793 \mathrm{~g} \mathrm{BaSO}$ b) $\left.0,02733 \mathrm{~g} \mathrm{BaSO}_{4}\right\} \quad$ Spülwasser $0,1444 \mathrm{~g} \mathrm{~S}$.

16. Mai 1907. a) $\left.0,01343 \mathrm{~g} \mathrm{BaSO}_{4}\right\}=0,0018 \mathrm{~g} \mathrm{~S}$. In $115 \mathrm{~cm} \mathrm{Urin}+85 \mathrm{ccm}$ b) $\left.0,01283 \mathrm{~g} \mathrm{BaSO}_{4}\right\} \quad$ Spülwasser $0,0720 \mathrm{~g} \mathrm{~S}$.

Mai 1907. a) $0,04087 \mathrm{~g} \mathrm{BaSO}_{4} \mid=0,0055 \mathrm{~g} \mathrm{~S}$. In $155 \mathrm{ccm}$ Urin $+45 \mathrm{ccm}$. b) $0,03953 \mathrm{~g} \mathrm{BaSO}_{4} \mathrm{f}$ Spülwasser $0,2200 \mathrm{~g} \mathrm{~S}$.

20. Mai 1907. a) $0,02563 \mathrm{~g} \mathrm{BaSO} 4\}=0,0085 \mathrm{~g} \mathrm{~S}$. In $150 \mathrm{ccm} \mathrm{Urin}+100 \mathrm{ccm}$. b) $\left.0,02613 \mathrm{~g} \mathrm{BaSO}_{4}\right\} \quad$ Spülwasser $0,1750 \mathrm{~g} \mathrm{~S}$.

22. Mai 1907. a) $\left.0,02723 \mathrm{~g} \mathrm{BaSO} \mathrm{g}_{4}\right\}=0,0038 \mathrm{~g} \mathrm{~S}$. In $50 \mathrm{ccm}$ Urin $+80 \mathrm{ccm}$ b) $\left.0,02763 \mathrm{~g} \mathrm{BaSO}_{4}\right\} \quad$ Spülwasser $0,0988 \mathrm{~g} \mathrm{~S}$.

24. Mai 1907. a) $\left.0,03793 \mathrm{~g} \mathrm{BaSO}_{ \pm}\right\}=0,0052 \mathrm{~g} \mathrm{~S}$. In $50 \mathrm{ccm}$ Urin $+105 \mathrm{ccm}$ b) $\quad-\quad$ Spülwasser $0,1716 \mathrm{~g} \mathrm{~s}$.

26. Mai 1907. a) $0,05838 \mathrm{~g} \mathrm{BaSO}_{4} \mathrm{l}=0,0072 \mathrm{~g} \mathrm{~S}$. In $80 \mathrm{ccm} \mathrm{Urin}+85 \mathrm{ccm}$ b) $\left.0,05168 \mathrm{~g} \mathrm{BaSO}_{4}\right\} \quad$ Spülwasser $0,2376 \mathrm{~g} \mathrm{~S}$.

27. Mai 1907. a) $0,01373 \mathrm{~g} \mathrm{BaSO}_{4} \mid=0,0018 \mathrm{~g} \mathrm{~S}$. In $25 \mathrm{ccm}$ Urin $+75 \mathrm{ccm}$. b) $\left.0,01283 \mathrm{~g} \mathrm{BaSO}_{4}\right\} \quad$ Spülwasser $0,0360 \mathrm{~g} \mathrm{~S}$.

Kaninchen Nr. 15 und 16.

9. Mai 1907. a) $\left.0,0430 \mathrm{~g} \mathrm{BaSO}_{4}\right\}=0,0058 \mathrm{~g} \mathrm{~S}$. In $410 \mathrm{ccm} \mathrm{Urin}+95 \mathrm{ccm}$ b) $\left.0,0425 \mathrm{~g} \mathrm{BaSO}_{4}\right\} \quad$ Spülwasser $0,2903 \mathrm{~g} \mathrm{~S}$.

10. Mai 1907. a) $\left.0,03943 \mathrm{~g} \mathrm{BaSO}_{4}\right\}=0,0056 \mathrm{~g}$ S. In $750+10 \mathrm{ccm}$ Urin + b) $\left.0,04013 \mathrm{~g} \mathrm{BaSO}_{4}\right\} \quad$ Spülwasser $0,4256 \mathrm{~g} \mathrm{~S}$.

12. Mai 1907. a) $\left.0,05723 \mathrm{~g} \mathrm{BaSO}_{4}\right\}=0,0079 \mathrm{~g} \mathrm{~S}$. In $260+50 \mathrm{ccm}$ Urin + b) $\left.0,05803 \mathrm{~g} \mathrm{BaSO}_{4}\right\} \quad$ Spülwasser $0,2449 \mathrm{~g} \mathrm{~S}$.

14. Mai 1907. a) $\left.0,03043 \mathrm{~g} \mathrm{BaSO}_{4}\right\}=0,0041 \mathrm{~g}$ S. In $150+60 \mathrm{cem}$ Urin + b) $\left.0,02963 \mathrm{~g} \mathrm{BaSO}_{4}\right\} \quad$ Spülwasser $0,1722 \mathrm{~g} \mathrm{~S}$. 
16. Mai 1907. a) $\left.0,01183 \mathrm{~g} \mathrm{BaSO}_{4}\right\}=0,0017 \mathrm{~g} \mathrm{~S}$. In $140+155 \mathrm{ccm}$ Urin + b) $\left.0,01233 \mathrm{~g} \mathrm{BaSO}_{4}\right\} \quad$ Spülwasser $0,1003 \mathrm{~g} \mathrm{~S}$.

18. Mai 1907. a) $\left.0,03143 \mathrm{~g} \mathrm{BaSO}_{4}\right\}=0,0043 \mathrm{~g}$ S. In $100+180 \mathrm{ccm}$ Urin + b) - Spülwasser $0,1978 \mathrm{~g} \mathrm{~S}$.

20. Mai 1907. a) $0,03163 \mathrm{~g} \mathrm{BaSO}_{4} \mathrm{l}=0,0044 \mathrm{~g} \mathrm{~S}$. In $100+150 \mathrm{~cm} \mathrm{Urin}+$ b) $0,08228 \mathrm{~g} \mathrm{BaSO}_{4} \mathrm{~J} \quad$ Spülwasser $0,2200 \mathrm{~g} \mathrm{~S}$.

22. Mai 1907. a) $\left.0,04483 \mathrm{~g} \mathrm{BaSO}_{4}\right\}=0,0061 \mathrm{~g} \mathrm{~S}$. In $100+110 \mathrm{ccm}$ Urin + b) $\left.0,04473 \mathrm{~g} \mathrm{BaSO}_{4}\right\} \quad$ Spülwasser $0,2562 \mathrm{~g} \mathrm{~S}$.

24. Mai 1907. a) $\left.0,05303 \mathrm{~g} \mathrm{BaSO}_{4}\right\}=0,0072 \mathrm{~g} \mathrm{~S}$. In $50+95 \mathrm{~cm}$ Urin + b) $\left.0,05233 \mathrm{~g} \mathrm{BaSO}_{4}\right\} \quad$ Spülwasser $0,2088 \mathrm{~g} \mathrm{~S}$.

26. Mai 1907. a) $\left.0,08353 \mathrm{~g} \mathrm{BaSO}_{4}\right\}=0,0045 \mathrm{~g} \mathrm{~S}$. In $50+75 \mathrm{ccm}$ Urin + b) $\left.0,08263 \mathrm{~g} \mathrm{BaSO}_{4}\right\} \quad$ Spülwasser $0,1125 \mathrm{~g} \mathrm{~S}$.

28. Mai 1907. a) $\left.0,02533 \mathrm{~g} \mathrm{BaSO}_{4}\right\}=0,0035 \mathrm{~g} \mathrm{~S}$. In $50+110 \mathrm{ccm}$ Urin + b) $\left.0,02583 \mathrm{~g} \mathrm{BaSO}_{4}\right\} \quad$ Spülwasser $0,1120 \mathrm{~g} \mathrm{~S}$.

30. Mai 1907. a) $0,03853 \mathrm{~g} \mathrm{BaSO}+4=0,0047 \mathrm{~g} \mathrm{~S}$. In $50+90 \mathrm{ccm}$ Urin + b) $0,03553 \mathrm{~g} \mathrm{BaSO}_{4} \quad$ Spülwasser $0,1310 \mathrm{~g} \mathrm{~S}$.

\section{Kaninchen Nr. 17 und 18.}

9. Mai 1907. a) $0,02823 \mathrm{~g} \mathrm{BaSO} 4\}=0,0089 \mathrm{~g} \mathrm{~S}$. In $455+75 \mathrm{ccm}$ Urin + b) $\left.0,02893 \mathrm{~g} \mathrm{BaSO}_{4}\right\} \quad$ Spülwasser $0,2078 \mathrm{~g} \mathrm{~S}$.

10. Mai 1907. a) $\left.0,04063 \mathrm{~g} \mathrm{BaSO}_{4}\right\}=0,0056 \mathrm{~g} \mathrm{~S}$. In $610+50 \mathrm{ccm}$ Urin +
b) 0,0
Spülwasser $0,3976 \mathrm{~g} \mathrm{~S}$.

12. Mai 1907. a) $\left.0,04473 \mathrm{~g} \mathrm{BaSO}_{4}\right\}=0,0061 \mathrm{~g} \mathrm{~S}$. In $290+100 \mathrm{ccm}$ Urin + b) $\left.0,04453 \mathrm{~g} \mathrm{BaSO}_{4}\right\} \quad$ Spülwasser $0,2379 \mathrm{~g} \mathrm{~S}$.

14. Mai 1907. a) $\left.0,03033 \mathrm{~g} \mathrm{BaSO}_{4}\right\}=0,0042 \mathrm{~g}$ S. In $150+50 \mathrm{ccm}$ Urin + b) $\left.0,03083 \mathrm{~g} \mathrm{BaSO}_{4}\right\} \quad$ Spülwasser $0,1680 \mathrm{~g} \mathrm{~S}$.

16. Mai 1907. a) $\left.0,03059 \mathrm{~g} \mathrm{BaSO} \mathrm{B}_{4}\right\}=0,0042 \mathrm{~g} \mathrm{~S}$. In $160+105 \mathrm{~cm}$ Urin + a) $\left.0,03123 \mathrm{~g} \mathrm{BaSO}_{4}\right\} \quad$ Spülwasser $0,2226 \mathrm{~g} \mathrm{~S}$.

18. Mai 1907. a) $\left.0,06003 \mathrm{~g} \mathrm{BaSO}_{4}\right\}=0,0069 \mathrm{~g}$ S. In $165+95 \mathrm{ccm}$ Urin + b) - $\quad$ Spülwasser $0,3588 \mathrm{~g} \mathrm{~S}$.

20. Mai 1907. a) $\left.0,06193 \mathrm{~g} \mathrm{BaSO}_{4}\right\}=0,0085 \mathrm{~g}$ S. In $165+60 \mathrm{ccm}$ Urin + b) $\left.0,06283 \mathrm{~g} \mathrm{BaSO}_{4}\right\} \quad$ Spülwasser $0,3825 \mathrm{~g} \mathrm{~S}$.

22. Mai 1907. a) $\left.0,03773 \mathrm{~g} \mathrm{BaSO}_{4}\right\}=0,0051 \mathrm{~g} \mathrm{~S}$. In $75+75 \mathrm{ccm}$ Urin + b) $\left.0,03733 \mathrm{~g} \mathrm{BaSO}_{4}\right\} \quad$ Spüiwasser $0,1530 \mathrm{~g} \mathrm{~s}$.

24. Mai 1907. a) $\left.0,03353 \mathrm{~g} \mathrm{BaSO}_{4}\right\}=0,0047 \mathrm{~g} \mathrm{~S}$. In $70+50 \mathrm{ccm} \mathrm{Urin}+$ b) $\left.0,03443 \mathrm{~g} \mathrm{BaSO}_{4}\right\} \quad$ Spulwasser $0,1175 \mathrm{~g} \mathrm{~S}$.

26. Mai 1907. a) $\left.0,03853 \mathrm{~g} \mathrm{BaSO}_{4}\right\}=0,0043 \mathrm{~g} \mathrm{~S}$. In $55+70 \mathrm{ccm}$ Urin + b) - $\quad$ Spülwasser 0,1075 g S.

28. Mai 1907. a) $\left.0,03208 \mathrm{~g} \mathrm{BaSO}_{4}\right\}=0,0044 \mathrm{~g} \mathrm{~S}$. In $50+85 \mathrm{~cm} \mathrm{Urin}+$ b) - Spülwasser $0,1188 \mathrm{~g} \mathrm{~S}$.

30. Mai 1907. a) $\left.0,04273 \mathrm{~g} \mathrm{BaSO}_{4}\right\}=0,0058 \mathrm{~g} \mathrm{~S}$. In $90+95 \mathrm{ccm}$ Urin + b) $\left.0,04.173 \mathrm{~g} \mathrm{BaSO}_{4}\right\} \quad$ Spülwasser $0,2146 \mathrm{~g} \mathrm{~S}$.

Die analytischen Belege der Stickstoffbestimmungen sollen, weil die Zahlen der Analysen doch keine anderen Schlïsse als die S-Bestimmungen geben, nicht in extenso aufgeführt werden. 


\section{Serie IV.}

\section{Stickstoffbestimmungen in 2 ecm Urin.}

7. Oktober 1907.

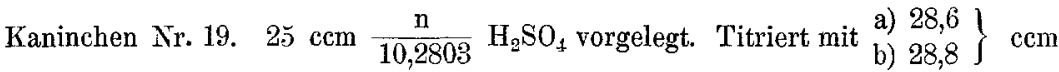
$\frac{\mathrm{n}}{\mathrm{x}} \mathrm{NaOH}=22,3 \mathrm{ccm} \frac{\mathrm{n}}{10,2803} \mathrm{H}_{2} \mathrm{SO}_{4}$. Gebunden $2,7 \mathrm{ccm} \frac{\mathrm{n}}{10,2803} \mathrm{H}_{2} \mathrm{SO}_{4}=$ $0,00367686 \mathrm{~g} \mathbf{N}$. In $600 \mathrm{ccm}$ Urin + Spülwasser $\mathbf{1 , 1 0 3 0 6 ~ g ~} \mathbf{N}$.

Kaninchen $\mathrm{Nr} .20 .12 \mathrm{ccm} \frac{\mathrm{n}}{10,2803} \mathrm{H}_{2} \mathrm{SO}_{4}$ vorgelegt. Titriert mit $\left.\begin{array}{l}\text { a) } 12,3 \\ \text { b) } 12,5\end{array}\right\} \mathrm{ccm}$ $\frac{\mathrm{n}}{\mathrm{x}} \mathrm{NaOH}=9,8 \mathrm{~cm} \frac{\mathrm{n}}{10,2803} \mathrm{H}_{2} \mathrm{SO}_{4}$. Gebunden $2,2 \mathrm{ccm} \frac{\mathrm{n}}{10,2803} \mathrm{H}_{2} \mathrm{SO}_{4}=$ $0,002996 \mathrm{~g} \mathrm{~N}$. In $490 \mathrm{ccm}$ Urin + Spülwasser 0,7850 g $\mathbf{~}$.

Kaninchen Nr. 21. $25 \mathrm{ccm} \frac{\mathrm{n}}{10,2803} \mathrm{H}_{2} \mathrm{SO}_{4}$ vorgelegt. Titriert mit a) 27,6$\}$ b) 27,8$\} \mathrm{ccm}$ $\frac{\mathrm{n}}{\mathrm{x}} \mathrm{NaOH}=22 \mathrm{ccm} \frac{\mathrm{n}}{10,2803} \mathrm{H}_{2} \mathrm{SO} 0_{\downarrow}$. Gebunden $3,0 \mathrm{ccm} \frac{\mathrm{n}}{10,2803} \mathrm{H}_{2} \mathrm{SO}_{4}=$ $0,004086 \mathrm{~g} \mathrm{~N}$. In $500 \mathrm{~cm}$ Urin + Spülwasser 1,0213 $\mathrm{g} \mathbf{~}$.

Kaninchen Nr. 22. $12 \mathrm{ccm} \frac{\mathrm{n}}{10,2803} \mathrm{H}_{9} \mathrm{SO}_{4}$ vorgelegt. Titriert mit $\left.\begin{array}{l}\text { a) } 12,6 \\ \text { b) } 12,8\end{array}\right\} \mathrm{ccm}$ $\frac{\mathrm{n}}{\mathrm{x}} \mathrm{NaOH}$. Mittel $12,7 \frac{\mathrm{n}}{\mathrm{x}} \mathrm{NaOH}=10 \mathrm{ccm} \frac{\mathrm{n}}{10,2803} \mathrm{H}_{2} \mathrm{SO}_{4} . \quad$ Gebunden $2 \mathrm{ccm} \frac{\mathrm{n}}{10,2803} \mathrm{H}_{2} \mathrm{SO}_{4}=0,002724 \mathrm{~g}$ N. In $565 \mathrm{ccm}$ Urin + Spülwasser $0,7694 \mathrm{~g}$ N.

Kaninchen Nr. 23. $25 \mathrm{ccm} \frac{\mathrm{n}}{10,2808} \mathrm{H}_{2} \mathrm{SO}_{4}$ vorgelegt. Titriert mit $\left.\begin{array}{l}\text { a) } 27,1 \\ \text { b) } 27,1\end{array}\right\} \mathrm{ccm}$ $\frac{\mathrm{n}}{\mathrm{x}} \mathrm{NaOH}=21,5 \mathrm{ccm} \frac{\mathrm{n}}{10,2803} \mathrm{H}_{2} \mathrm{SO}_{4}$. Gebunden $3,5 \mathrm{ccm} \frac{\mathrm{n}}{10,2803} \mathrm{H}_{2} \mathrm{SO}_{4}=$ $0,0047663 \mathrm{~g} \mathrm{~N}$. In $415 \mathrm{ccm}$ Urin + Spülwasser 0,9890 g $\mathbf{~}$.

9. Oktober 1907.

Kaninchen Nr. 19. $12 \mathrm{ccm} \frac{\mathrm{n}}{10,2808} \mathrm{H}_{2} \mathrm{SO}_{4}$ vorgelegt. Titriert mit $\left.\begin{array}{l}\text { a) } 11,7 \\ \text { b) } 11,9\end{array}\right\} \mathrm{ccm}$ $\frac{\mathrm{n}}{\mathrm{x}} \mathrm{NaOH}$. Mittel $11,8 \mathrm{ccm} \frac{\mathrm{n}}{\mathrm{x}} \mathrm{NaOH}=9,4 \mathrm{ccm} \frac{\mathrm{n}}{10,2803} \mathrm{H}_{2} \mathrm{SO}_{4}$. Gebunden $2,6 \mathrm{ccm} \frac{\mathrm{n}}{10,2803} \mathrm{H}_{2} \mathrm{SO}_{4}=0,002541 \mathrm{~g} \mathrm{~N}$. In $465 \mathrm{ccm}$ Urin + Spülwasser $0,6035 \mathrm{~g} \mathrm{~N}$.

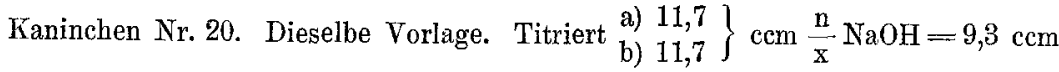
$\frac{\mathrm{n}}{10,2803} \mathrm{H}_{2} \mathrm{SO}_{4}$. Gebunden $2,7 \mathrm{ccm} \frac{\mathrm{n}}{10,2803} \mathrm{H}_{2} \mathrm{SO}_{4}=0,003677 \mathrm{~g}$ N. In $415 \mathrm{ccm}$ Urin + Spülwasser $0,7629 \mathrm{~g} \mathrm{X}$. 
Kaninchen Nr. 21. Dieselbe Vorlage. Titriert $\left.\begin{array}{l}\text { a) } 11,5 \\ \text { b) } 11,6\end{array}\right\} \operatorname{ccm} \frac{n}{x}$ NaOH. Mittel $11,55 \mathrm{ccm} \frac{\mathrm{n}}{\mathrm{x}} \mathrm{NaOH}=9,2 \mathrm{~cm} \frac{\mathrm{n}}{10,2803} \mathrm{H}_{2} \mathrm{SO}_{4}$. Gebunden $2,8 \mathrm{~cm} \frac{\mathrm{n}}{10,2808}$ $\mathrm{H}_{2} \mathrm{SO}_{4}=0,003813 \mathrm{~g} \mathrm{~N}$. In $455 \mathrm{ccm}$ Urin + Spülwasser $\mathbf{0 , 8 6 7 5} \mathbf{g} \mathbf{~}$.

Kaninchen Nr. 22. Dieselbe Vorlage. Titriert a) 11,5$\}$ b) 12,2$\}$ ccm $\frac{n}{x} \mathrm{NaOH}$. Mittel $11,85 \mathrm{ccm} \frac{\mathrm{n}}{\mathrm{x}} \mathrm{NaOH}=9,4 \mathrm{~cm} \frac{\mathrm{n}}{10,2803} \mathrm{H}_{2} \mathrm{SO}_{ \pm}$. Gebunden $2,6 \mathrm{ccm} \frac{\mathrm{n}}{10,2803}$ $\mathrm{H}_{2} \mathrm{SO}_{4}=0,003541 \mathrm{~g} \mathrm{~N}$. In $460 \mathrm{ccm}$ Urin + Spülwasser $\mathbf{0 , 8 1 4 6} \mathrm{g} \mathbf{~ N}$.

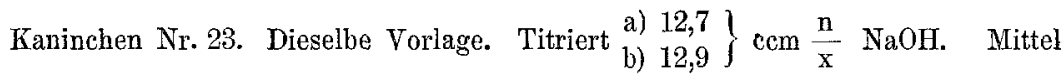
$12,8 \mathrm{ccm} \frac{\mathrm{n}}{\mathrm{x}} \mathrm{NaOH}=10,2 \mathrm{ccm} \frac{\mathrm{n}}{10,2803} \mathrm{H}_{2} \mathrm{SO}_{4}$. Gebunden $1,8 \mathrm{ccm} \frac{\mathrm{n}}{10,2808}$ $\mathrm{H}_{2} \mathrm{SO}_{4}=0,002451 \mathrm{~g} \mathrm{~N}$. In $545 \mathrm{ccm}$ Urin + Spülwasser $\mathbf{0 , 6 6 7 9} \mathbf{g} \mathbf{~}$.

11. Oktober 1907. $25 \mathrm{ccm} \frac{\mathrm{n}}{10,2808} \mathrm{H}_{2} \mathrm{SO}_{4}$ vorgelegt.

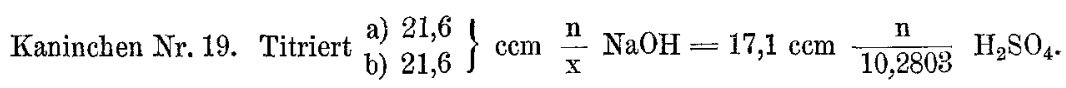
Gebunden $7,9 \mathrm{~cm} \frac{\mathrm{n}}{10,2803} \mathrm{H}_{4} \mathrm{SO}_{4}=0,0108 \mathrm{~g} \mathrm{~N}$. In $250 \mathrm{ccm}$ Urin + Spülwasser $1,3448 \mathrm{~g} \mathbf{~}$.

Kaninchen Nr. 20. Titriert $\left.\begin{array}{l}\text { a) } 18,0 \\ \text { b) } 18,0\end{array}\right\} \mathrm{ccm} \frac{\mathrm{n}}{\mathrm{x}} \mathrm{NaOH}=14,3 \mathrm{~cm} \frac{\mathrm{n}}{10,2803} \mathrm{H}_{2} \mathrm{SO}_{4}$. Gebunden $10,7 \mathrm{~cm} \frac{\mathrm{n}}{10,2803} \quad \mathrm{H}_{2} \mathrm{SO}_{4}=0,0146 \mathrm{~g} \mathrm{~N}$. In $160 \mathrm{ccm}$ Urin + Spülwasser $1,1657 \mathrm{~g} \mathbf{N}$.

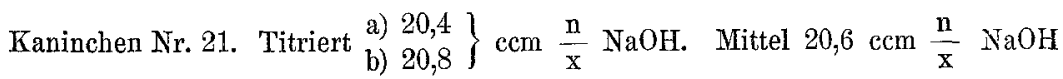
$=16,3 \mathrm{~cm} \frac{\mathrm{n}}{10,2803} \mathrm{H}_{2} \mathrm{SO}_{4}$. Gebunden $8,7 \mathrm{ccm} \frac{\mathrm{n}}{10,2803} \mathrm{H}_{2} \mathrm{SO}_{4}=0,0184 \mathrm{~g} \mathrm{~N}$. In $205 \mathrm{ccm}$ Urin + Spülwasser $1,2114 \mathrm{~g} \mathrm{~N}$.

Kaninchen Nr. 22. Titriert a) 27,4$\}$ b) 27,6$\}$ Mittel $27,5 \mathrm{ccm} \frac{\mathrm{n}}{\mathrm{x}} \mathrm{NaOH}=21,8 \mathrm{~cm} \frac{\mathrm{n}}{10,2808}$ $\mathrm{H}_{2} \mathrm{SO}_{4}$. Gebunden $3,2 \mathrm{ccm} \frac{\mathrm{n}}{10,2803} \mathrm{H}_{2} \mathrm{SO}_{4}=0,0044 \mathrm{~g} \mathrm{~N}$. In $270 \mathrm{ccm}$ Urin + Spülwasser $\mathbf{0 , \mathbf { a } 8 8 3} \mathrm{g} \mathbf{N}$.

Kaninchen Nr. 23. Titriert a) 24,5$\}$ b) 24,4$\}$ Mittel $24,45 \mathrm{ccm} \frac{\mathrm{n}}{\mathrm{x}} \mathrm{NaOH}=19,4 \mathrm{~cm}$ $\frac{\mathrm{n}}{10,2803} \mathrm{H}_{2} \mathrm{SO}_{4}$. Gebunden $5,6 \mathrm{ccm} \frac{\mathrm{n}}{10,2803} \mathrm{H}_{2} \mathrm{SO}_{4}=0,0076 \mathrm{~g} \mathrm{~N}$. In $185 \mathrm{ccm}$ Urin + Spülwasser $0,4767 \mathrm{~g} \mathbf{N}$. 
Der Einfluss des Alkohols am Hungertier auf Lebensdaner etc.

13. Oktober 1907. 25- ccm $\frac{\mathrm{n}}{10,2803} \mathrm{H}_{2} \mathrm{SO}_{4}$ vorgelegt.

Kaninchen Nr. 19. Titriert a) 17,6 b) 17,5$\}$ Mittel $17,55 \mathrm{ccm} \frac{\mathrm{n}}{\mathrm{x}} \mathrm{NaOH}=13,9 \mathrm{ccm}$ $\frac{\mathrm{n}}{10,2803} \mathrm{H}_{2} \mathrm{SO}_{4}$. Gebunden $11,1 \mathrm{ccm} \frac{\mathrm{n}}{10,2803} \mathrm{H}_{2} \mathrm{SO}_{4}=0,01512 \mathrm{~g} \mathrm{~N}$. In $190 \mathrm{ccm}$ Urin + Spülwasser $\mathbf{1 , 4 3 6 4}$ g $\mathbf{~}$.

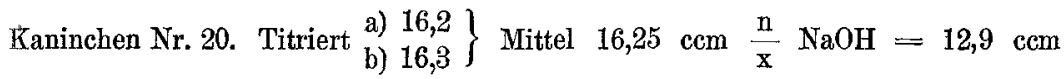
$\frac{\mathrm{n} .}{10,2803} \mathrm{H}_{2} \mathrm{SO}_{4}$. Gebunden $12,1 \mathrm{ccm} \frac{\mathrm{n}}{10,2803} \mathrm{H}_{2} \mathrm{SO}_{4}=0,0165 \mathrm{~g} \mathrm{~N} . \quad$ In $165 \mathrm{ccm}$ Urin + Spülwasser 1,3613 g $\mathbf{~}$.

Kaninchen Nr. 21. Titriert a) 17,0$\}$ b) 17,1$\}$ Mittel $17,05 \mathrm{ccm} \frac{\mathrm{n}}{\mathrm{x}} \mathrm{NaOH}=13,5 \mathrm{ccm}$ $\frac{\mathrm{n}}{10,2803} \mathrm{H}_{2} \mathrm{SO}_{4}$. Gebunden $11,5 \mathrm{ccm} \frac{\mathrm{n}}{10,2803} \mathrm{H}_{2} \mathrm{SO}_{4}=0,0157 \mathrm{~g}$ N. In $185 \mathrm{ccm}$ Urin + Spülwasser 1,45225 $\mathrm{g} \mathrm{N}$.

Kaninchen Nr. 22. Titriert a) 15,9$\}$ b) 15,4$\}$ Mittel $15,65 \mathrm{ccm} \frac{\mathrm{n}}{\mathrm{x}} \mathrm{NaOH}=12,4 \mathrm{ccm}$ $\frac{\mathrm{n}}{10,2803} \mathrm{H}_{2} \mathrm{SO}_{4}$. Gebunden $12,6 \mathrm{ccm} \frac{\mathrm{n}}{10,2803} \mathrm{H}_{2} \mathrm{SO}_{4}=0,0172 \mathrm{~g}$ N. In $180 \mathrm{ccm}$ Urin + Spülwasser 1,5480 $\mathrm{g} \mathbf{~ N}$.

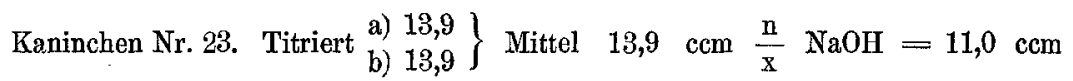
$\frac{\mathrm{n}}{10,2808} \mathrm{H}_{2} \mathrm{SO}_{4}$. Gebunden $14,0 \mathrm{ccm} \frac{\mathrm{n}}{10,2803} \mathrm{H}_{2} \mathrm{SO}_{4}=0,0191 \mathrm{~g}$ N. In $195 \mathrm{ccm}$ Urin + Spülwasser $\mathbf{1 , 8 6 2 2} \mathrm{g} \mathbf{~}$.

15. Oktober 1907. $25 \mathrm{ccm} \frac{\mathbf{n}}{10,2803} \mathrm{H}_{2} \mathrm{SO}_{4}$ vorgelegt.

Kaninchen Nr. 19. Titriert $\left.\begin{array}{l}\text { a) } 15,3 \\ \text { b) } 15,3\end{array}\right\}$ Mittel $15,3 \mathrm{ccm} \frac{\mathrm{n}}{\mathrm{x}} \mathrm{NaOH}=12,1 \mathrm{ccm} \frac{\mathbf{n}}{10,2803}$ $\mathrm{H}_{2} \mathrm{SO}_{4}$. Gebunden $12,9 \mathrm{ccm} \frac{\mathrm{n}}{10,2803} \mathrm{H}_{2} \mathrm{SO}_{4}=0,0175 \mathrm{~g} \mathrm{~N}$. In $140 \mathrm{ccm} \mathrm{Urin}+$ Spülwasser $1,2250 \mathrm{~g}$ N.

Kaninchen Nr. 20. Titriert $\left.\begin{array}{l}\text { a) } 12,8 \\ \text { b) } 12,8\end{array}\right\}$ Mittel $12,8 \mathrm{~cm} \frac{\mathrm{n}}{\mathrm{x}} \mathrm{NaOH}=10,2 \mathrm{ccm} \frac{\mathrm{n}}{10,2803}$ $\mathrm{H}_{2} \mathrm{SO}_{4}$. Gebunden $14,8 \mathrm{ccm} \frac{\mathrm{n}}{10,2803} \mathrm{H}_{2} \mathrm{SO}_{4}=0,0201 \mathrm{~g} \mathrm{~N}$. In $130 \mathrm{ccm}$ Urin + Spülwasser 1,3065 g $\mathbf{N}$.

Kaninchen Nr. 21. Titriert $\left.\begin{array}{l}\text { a) } 14,8 \\ \text { b) } 14,7\end{array}\right\}$ Mittel 14,75 ccm $\frac{\mathbf{n}}{\mathrm{x}} \mathrm{NaOH}=11,7 \mathrm{ccm} \frac{\mathbf{n}}{10,2803}$ $\mathrm{H}_{2} \mathrm{SO}_{4}$. Gebunden $13,3 \mathrm{ccm} \frac{\mathrm{n}}{10,2803} \mathrm{H}_{2} \mathrm{SO}_{4}=0,0181 \mathrm{~g} \mathrm{~N}$. In $145 \mathrm{ccm}$ Urin + Spülwasser $1,3122 \mathrm{~g} \mathbf{N}$.

E. Pfl ŭger, Archiv für Physiologie. Bd. 127. 
Kaninchen Nr. 22. Titriert a) 13,1$\}$ b) 13,4$\}$ Mittel $13,25 \mathrm{ccm} \frac{\mathrm{n}}{\mathrm{x}} \mathrm{NaOH}=10,5 \mathrm{~cm} \frac{\mathrm{n}}{10,2808}$ $\mathrm{H}_{2} \mathrm{SO}_{4}$. Gebunden $14,5 \mathrm{~cm} \frac{\mathrm{n}}{10,2803} \mathrm{H}_{2} \mathrm{SO}_{4}=0,0197 \mathrm{~g} \mathrm{~N}$. In $145 \mathrm{ccm}$ Urin + Spülwasser 1,4282 $\mathrm{g}$ N.

Kaninchen Nr. 23. Titriert $\left.\begin{array}{l}\text { a) } 14,3 \\ \text { b) } 14,6\end{array}\right\} \operatorname{Mittel} 14,45 \mathrm{ccm} \frac{\mathrm{n}}{\mathrm{x}} \mathrm{NaOH}=11,5 \mathrm{ccm} \frac{\mathrm{n}}{10,2808}$ $\mathrm{H}_{2} \mathrm{SO}_{4}$. Gebunden $13,5 \mathrm{ccm} \frac{\mathrm{n}}{10,2803} \mathrm{H}_{2} \mathrm{SO}_{4}=0,0184 \mathrm{~g} \mathrm{~N}$. In $150 \mathrm{ccm}$ Urint Spülwasser 1,3800 $\mathrm{g}$ N.

17. Oktober 1907. $25 \mathrm{ccm} \frac{\mathrm{n}}{10,2803} \mathrm{H}_{2} \mathrm{SO}_{4}$ vorgelegt.

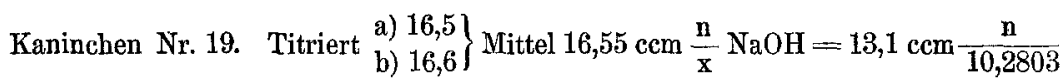
$\mathrm{H}_{2} \mathrm{SO}_{4}$. Gebunden $11,9 \mathrm{ccm} \frac{\mathbf{n}}{10,2808} \mathrm{H}_{2} \mathrm{SO}_{4}=0,0162 \mathrm{~g} \mathrm{~N}$. In $155 \mathrm{ccm}$ Urin + Spülwasser 1,2555 g N.

Kaninchen Nr. 20. Titriert $\left.\begin{array}{l}\text { a) } 11,4 \\ \text { b) } 11,5\end{array}\right\}$ Mittel 11,45 ccm $\frac{\mathrm{n}}{\mathrm{x}} \mathrm{NaOH}=9,1 \mathrm{ccm} \frac{\mathrm{n}}{10,2803}$ $\mathrm{H}_{2} \mathrm{SO}_{4}$. Gebunden $15,9 \mathrm{ccm} \frac{n}{10,2803} \mathrm{H}_{2} \mathrm{SO}_{4}=0,0216 \mathrm{~g} \mathrm{~N}$. In $110 \mathrm{ccm}$ Urin + Spülwasser $\mathbf{1 , 1 8 8 0} \mathrm{g} \mathbf{N}$.

Kaninchen Nr. 21. Titriert $\left.\begin{array}{l}\text { a) } 13,9 \\ \text { b) } 13,8\end{array}\right\} \operatorname{Mittel} 13,85 \mathrm{ccm} \frac{\mathrm{n}}{\mathrm{x}} \mathrm{NaOH}=11,1 \mathrm{ccm} \frac{\mathrm{n}}{10,2803}$ $\mathrm{H}_{2} \mathrm{SO}_{4}$. Gebunden $13,9 \mathrm{ccm} \frac{\mathrm{n}}{10,2803} \mathrm{H}_{2} \mathrm{SO}_{4}=0,0189 \mathrm{~g} \mathrm{~N}$. In $145 \mathrm{ccm}$ Urin+ Spü]wasser $1,3630 \mathrm{~g}$ N.

Kaninchen Nr. 22. Titriert $\left.\begin{array}{l}\text { a) } 14,7 \\ \text { b) } 14,2\end{array}\right\}$ Mittel $14,45 \mathrm{ccm} \frac{\mathrm{n}}{\mathrm{x}} \mathrm{NaOH}=11,5 \mathrm{ccm} \frac{\mathrm{n}}{10,2803}$ $\mathrm{H}_{2} \mathrm{SO}_{4}$. Gebunden $13,5 \mathrm{ccm} \frac{\mathrm{n}}{10,2830} \mathrm{H}_{2} \mathrm{SO}_{4}=0,0184 \mathrm{~g} \mathrm{~N}$. In $180 \mathrm{ccm}$ Urin+ Spülwasser $1,6560 \mathrm{~g} \mathrm{~N}$.

Kaninchen Nr. 23. Titriert a) 9,8$\}$ b) 9,9$\}$ Mittel $9,85 \mathrm{ccm} \frac{\mathrm{n}}{\mathrm{x}} \mathrm{NaOH}=7,8 \mathrm{ccm} \frac{\mathrm{n}}{10,2803}$ $\mathrm{H}_{2} \mathrm{SO}_{4}$. Gebunden $17,2 \mathrm{ccm} \frac{\mathrm{n}}{10,2803} \mathrm{H}_{2} \mathrm{SO}_{4}=0,0234 \mathrm{~g} \mathrm{~N}$. In $160 \mathrm{ccm}$ Urin + Spülwasser $1,8720 \mathrm{~g}$ N.

19. Oktober 1907. $25 \mathrm{ccm} \frac{\mathrm{n}}{10,2803} \mathrm{H}_{2} \mathrm{SO}_{4}$, vorgelegt.

Kaninchen Nr. 19. Titriert $\left.\begin{array}{l}\text { a) } 5,6 \\ \text { b) } 5,8\end{array}\right\}$ Mittel $5,7 \mathrm{ccm} \frac{\mathrm{n}}{\mathrm{x}} \mathrm{NaOH}=4,5 \mathrm{ccm} \frac{\mathrm{n}}{10,2803}$ $\mathrm{H}_{2} \mathrm{SO}_{4}$. Gebunden $21,5 \mathrm{ccm} \frac{\mathrm{n}}{10,2803} \mathrm{H}_{2} \mathrm{SO}_{4}=0,0293 \mathrm{~g} \mathrm{~N}$. In $155 \mathrm{ccm}$ Urin + Spülwasser 2,27075 g N. 
Der Einfluss des Alkohols am Hungertier auf Lebensdauer etc. $\quad 349$

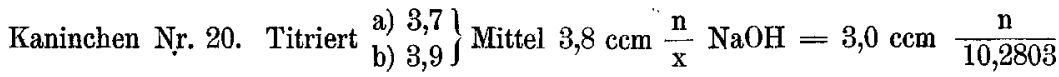
$\mathrm{H}_{2} \mathrm{SO}_{4}$. Gebunden $22 \mathrm{ccm} \frac{\mathrm{n}}{10,2803}-\mathrm{H}_{2} \mathrm{SO}_{4}=0,0300 \mathrm{~g} \mathrm{~N}$. In $140 \mathrm{ccm}$ Urin + Spülwasser 2,100 g N.

Kaninchen Nr. 21. Titriert $\left.\begin{array}{l}\text { a) } 9,1 \\ \text { b) } 9,0\end{array}\right\}$ Mittel $9,05 \mathrm{ccm} \frac{\mathrm{n}}{\mathrm{x}} \mathrm{NaOH}=7,2 \mathrm{ccm} \frac{\mathbf{n}}{10,2808}$ $\mathrm{H}_{2} \mathrm{SO}_{4}$. Gebunden $17,8 \mathrm{~cm} \frac{\mathrm{n}}{10,2803} \mathrm{H}_{2} \mathrm{SO}_{4}=0,0242 \mathrm{~g} \mathrm{~N}$. In $115 \mathrm{ccm}$ Urint Spülwasser 1,3915 g N.

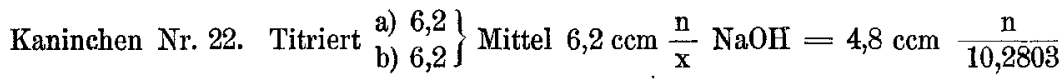
$\mathrm{H}_{2} \mathrm{SO}_{4}$. Gebunden $20,2 \mathrm{ccm} \frac{\mathrm{n}}{10,2803} \mathrm{H}_{2} \mathrm{SO}_{4}=0,0275 \mathrm{~g} \mathrm{~N}$. In $210 \mathrm{ccm}$ Urin + - Spülwasser 2,8875 g N.

Kaninchen Nr. 23. Titriert $\left.\begin{array}{l}\text { a) } 6,0 \\ \text { b) } 6,1\end{array}\right\}$ Mittel $6,05 \mathrm{~cm} \frac{\mathrm{n}}{\mathrm{x}} \mathrm{NaOH}=4,8 \mathrm{~cm} \frac{\mathbf{n}}{10,2803}$ $\mathrm{H}_{2} \mathrm{SO}_{4}$. Gebanden $21,2 \mathrm{ccm} \frac{\mathrm{n}}{10,2808} \mathrm{H}_{2} \mathrm{SO}_{4}=0,0289 \mathrm{~g} \mathrm{~N}$. In $125 \mathrm{ccm}$ Urint Spülwasser 1,80625 $\mathrm{g}$ N.

21. Oktober 1907. $25 \mathrm{ccm} \frac{\mathrm{n}}{10,2808} \mathrm{H}_{2} \mathrm{SO}_{4}$ vorgelegt.

Kaninchen Nr. 19. Titriert a) 17,1$\}$ b) 17,8$\}$ Mittel $17,05 \mathrm{ccm} \frac{\mathrm{n}}{\mathrm{x}} \mathrm{NaOH}=13,5 \mathrm{ccm} \frac{\mathrm{n}}{10,2803}$ $\mathrm{H}_{2} \mathrm{SO}_{4}$. Gebunden $11,5 \mathrm{ccm} \frac{\mathrm{n}}{10,2803} \mathrm{H}_{2} \mathrm{SO}_{4}=0,0157 \mathrm{~g} \mathrm{~N}$. In $125 \mathrm{ccm}$ Urin + Spülwasser 0,9812 g $\mathbf{N}$.

Kaninchen Nr. 20. Titriert $\left.\begin{array}{c}\text { a) } 0,2 \\ \text { b) } 0,2\end{array}\right\}$ Mittel $0,2 \mathrm{ccm} \frac{\mathrm{n}}{\mathrm{x}} \mathrm{NaOH}=0,2 \mathrm{ccm} \frac{\mathrm{n}}{10,2803}$ $\mathrm{H}_{2} \mathrm{SO}_{4}$. Gebunden $24,8 \mathrm{ccm} \frac{\mathrm{n}}{10,2803} \mathrm{H}_{2} \mathrm{SO}_{4}=0,0338 \mathrm{~g} \mathrm{~N}$. In $140 \mathrm{ccm}$ Urin + Spülwasser 2,3660 g $\mathbf{N}$.

Kaninchen Nr. 21. Titriert $\left.\begin{array}{l}\text { a) } 8,8 \\ \text { b) } 8,6\end{array}\right\}$ Mittel $8,7 \mathrm{ccm} \frac{\mathrm{n}}{\mathrm{x}} \mathrm{NaOH}=6,9 \mathrm{~cm} \frac{\mathrm{n}}{10,2803}$ $\mathrm{H}_{2} \mathrm{SO}_{4}$. Gebunden $18,1 \mathrm{ccm} \frac{\mathrm{n}}{10,2803} \mathrm{H}_{2} \mathrm{SO}_{4}=0,0240 \mathrm{~g} \mathrm{~N}$. In $125 \mathrm{ccm}$ Urin + Spülwasser 1,5373 $\mathrm{g} \mathbf{N}$.

23. Oktober 1907. $30 \mathrm{ccm} \frac{\mathrm{n}}{10,2803} \mathrm{H}_{2} \mathrm{SO}_{4}$ vorgelegt.

Kaninchen Nr. 20. Titriert $\left.\begin{array}{l}\text { a) } 6,5 \\ \text { b) } 6,8\end{array}\right\}$ Mittel $6,65 \mathrm{ccm} \frac{\mathrm{n}}{\mathrm{x}} \mathrm{NaOH}=5,3 \mathrm{ccm} \frac{\mathrm{n}}{10,2803}$ $\mathrm{H}_{2} \mathrm{SO}_{4}$. Gebunden $24,7 \mathrm{ccm} \frac{\mathrm{n}}{10,2803} \mathrm{H}_{2} \mathrm{SO}_{4}=0,0336 \mathrm{~g} \mathrm{~N}$. In $150 \mathrm{ccm}$ Urin + Spülwasser 2,5368 $\mathrm{g} \mathbf{N}$. 
Kaninchen Nr. 21. Titriert $\left.\begin{array}{l}\text { a) } 15,7 \\ \text { b) } 15,6\end{array}\right\}$ Mittel $15,65 \mathrm{ccm} \frac{\mathrm{n}}{\mathrm{x}} \mathrm{NaOH}=12,4 \mathrm{ccm} \frac{\mathrm{n}}{10,2803}$ $\mathrm{H}_{2} \mathrm{SO}_{4}$. Gebunden $17,6 \mathrm{ccm} \frac{\mathrm{n}}{10,2803} \mathrm{H}_{2} \mathrm{SO}_{4}=0,0240 \mathrm{~g} \mathrm{~N}$. In $165 \mathrm{ccm}$ Urin + Spülwasser 1,9800 g N.

\section{Stickstoffbestimmungen im Kot.}

9. Oktober 1907. $25 \mathrm{ccm} \frac{\mathrm{n}}{10,2803} \mathrm{H}_{2} \mathrm{SO}_{4}$ vorgelegt.

Kaninchen Nr. 19. 1 g Kot mit $20 \mathrm{ccm} \mathrm{H}_{2} \mathrm{SO}_{4}$ zerstört. Titriert mit $20,6 \mathrm{~cm}$ $\frac{\mathrm{n}}{\mathrm{x}} \mathrm{NaOH}=16,3 \mathrm{ccm} \frac{\mathrm{n}}{10,2803} \mathrm{H}_{2} \mathrm{SO}_{4}$. Gebunden $8,7 \mathrm{~cm} \frac{\mathrm{n}}{10,2808} \mathrm{H}_{2} \mathrm{SO}_{4}=$ $0,01184866 \mathrm{~g}$ N. In $17,3 \mathrm{~g}$ Kot $\mathbf{0 , 1 9 5 0} \mathrm{g} \mathbf{N}$.

Kaninchen Nr. 20. Ebenso. Titriert mit $29,4 \mathrm{ccm} \frac{\mathrm{n}}{\mathrm{x}} \mathrm{NaOH}=23,3 \mathrm{ccm} \frac{\mathrm{n}}{10,2803}$ $\mathrm{H}_{2} \mathrm{SO}_{4}$. Gebunden $1,7 \mathrm{~cm} \frac{\mathrm{n}}{10,2808} \mathrm{H}_{2} \mathrm{SO}_{4}=0,0021406 \mathrm{~g} \mathrm{~N}$. In 1,4 $\mathrm{g}$ Kot $0,0032 \mathrm{~g} \mathbf{~}$.

Kaninchen Nr. 21. Ebenso. Titriert $23,4 \mathrm{ccm} \frac{\mathrm{n}}{\mathrm{x}} \mathrm{NaOH}=18,6 \mathrm{ccm} \frac{\mathrm{n}}{10,2803} \mathrm{H}_{2} \mathrm{SO}_{4}$. Gebunden $6,4 \mathrm{ccm} \frac{\mathrm{n}}{10,2803} \mathrm{H}_{2} \mathrm{SO}_{4}=0,00871552 \mathrm{~g} \mathrm{~N}$. In $31 \mathrm{~g}$ Kot 0,2702 $\mathrm{g}$ N: Kaninchen Nr. 22. Ebenso. Titriert 27,7 $\mathrm{ccm} \frac{\mathrm{n}}{\mathrm{x}} \mathrm{NaOH}=22,0 \mathrm{~cm} \frac{\mathrm{n}}{10,2803} \mathrm{H}_{2} \mathrm{SO}_{4}$. Gebunden $3,0 \mathrm{ccm} \frac{\mathbf{n}}{10,2803} \mathrm{H}_{2} \mathrm{SO}_{4}=0,0040854 \mathrm{~g}$ N. In $20,9 \mathrm{~g} \operatorname{Kot} \mathbf{0 , 0 8 4 4} \mathrm{g} \mathbf{N}$. Kaninchen Nr. 23. Ebenso. Titriert $22,0 \mathrm{ccm} \frac{\mathrm{n}}{\mathrm{x}} \mathrm{NaOH}=17,4 \mathrm{ccm} \frac{\mathbf{n}}{10,2803} \mathrm{H}_{2} \mathrm{SO}_{4}$. Gebunden $7,6 \mathrm{ccm} \frac{\mathrm{n}}{10,2803} \mathrm{H}_{2} \mathrm{SO}_{4}=0,01034968 \mathrm{~g} \cdot \mathrm{N}$. In $19,7 \mathrm{~g}$ Kot $0,2089 \mathrm{~g} \mathrm{~N}$.

11. Oktober 1907. $25 \mathrm{ccm} \frac{\mathrm{n}}{10,2803} \mathrm{H}_{2} \mathrm{SO}_{4}$ vorgelegt.

Kaninchen Nr. 19. 1 g Kot zerstört. Titriert $21,9 \mathrm{ccm} \frac{\mathrm{n}}{\mathrm{x}} \mathrm{NaOH}=17,4 \mathrm{ccm} \frac{\mathbf{n}}{10,2803}$ $\mathrm{H}_{2} \mathrm{SO}_{4}$. Gebunden $7,6 \mathrm{ccm} \frac{\mathrm{n}}{10,2803} \mathrm{H}_{2} \mathrm{SO}_{4}=0,01034968 \mathrm{~g} \mathrm{~N}$. In $5,3 \mathrm{~g} \mathrm{Kot}$ $0,0548 \mathrm{~g} N$.

Kaninchen Nr. 20. Ebenso. Titriert 24,2 ccm $\frac{\mathrm{n}}{\mathrm{x}} \mathrm{NaOH}=19,2 \mathrm{~cm} \frac{\mathrm{n}}{10,2803} \mathrm{H}_{2} \mathrm{SO}_{4}$. Gebunden $5,8 \mathrm{ccm} \frac{\mathrm{n}}{10,2803} \mathrm{H}_{2} \mathrm{SO}_{4}=0,00789844 \mathrm{~g} \mathrm{~N}$. In $34,3 \mathrm{~g}$ Kot $0,9709 \mathrm{~g} \mathbf{N}$. 
Der Einfluss des Alkohols am Hungertier anf Lebensdauer etc.

Kaninchen Nr. 21. Ebenso. Titriert 23,0 $\mathrm{ccm} \frac{\mathrm{n}}{\mathrm{x}} \mathrm{NaOH}=18,2 \mathrm{ccm} \frac{\mathrm{n}}{10,2808} \mathrm{H}_{2} \mathrm{SO}_{4}$. Gebinden $6,8 \mathrm{~cm} \frac{\mathrm{n}}{10,2803} \mathrm{H}_{2} \mathrm{SO}_{4}=0,00926024^{\circ} \mathrm{g} \mathrm{N}$. In 23,2 g Kot $0,2148 \mathrm{~g}$ N.

Kaninchen Nr. 22. Ebenso. Titriert 21,1 ccm $\frac{n}{x} \mathrm{NaOH}=16,7 \mathrm{ccm} \frac{\mathrm{n}}{10,2803} \mathrm{H}_{2} \mathrm{SO}_{4}$. Gebunden $8,3 \mathrm{ccm} \frac{\mathrm{n}}{10,2803} \mathrm{H}_{2} \mathrm{SO}_{4}=0,01130294 \mathrm{~g} \mathrm{~N}$. In $27,1 \mathrm{~g}$ Kot 0,3063 g N.

Kaninchen Nr. 23. Ebenso. Titriert 16,7 ccm $\frac{\mathrm{n}}{\mathrm{x}} \mathrm{NaOH}=13,3 \mathrm{ccm} \frac{\mathrm{n}}{10,2803} \mathrm{H}_{2} \mathrm{SO}_{4}$. Gebunden $11,7 \mathrm{ccm} \frac{\mathrm{n}}{10,2803} \quad \mathrm{H}_{2} \mathrm{SO}_{4}=0,01593306 \mathrm{~g}$ N. In 25,5 g Kot $0,4063 \mathrm{~g}$ N.

13. Oktober 1907. $25 \mathrm{ccm} \frac{\mathrm{n}}{10,2803} \mathrm{H}_{2} \mathrm{SO}_{4}$ vorgelegt.

Kaninchen Nr. 19. $1 \mathrm{~g}$ Kot durch $\mathrm{H}_{2} \mathrm{SO}_{4}$ zerstört. Titriert $15,2 \mathrm{ccm} \frac{\mathrm{n}}{\mathrm{x}} \mathrm{NaOH}=$ $12,1 \mathrm{ccm} \frac{\mathrm{n}}{10,2808} \mathrm{H}_{2} \mathrm{SO}_{4}$. Gebunden $12,9 \mathrm{ccm} \frac{\mathrm{n}}{10,2803} \mathrm{H}_{2} \mathrm{SO}_{4}=0,0176 \mathrm{~g} \mathrm{~N}$. In $2,26 \mathrm{~g}$ Kot $0,0388 \mathrm{~g} \mathrm{~N}$.

Kaninchen Nr. 22. Ebenso. Titriert $12 \mathrm{ccm} \frac{\mathrm{n}}{\mathrm{x}} \mathrm{NaOH}=9,5 \mathrm{~cm} \frac{\mathrm{n}}{10,2803} \mathrm{H}_{2} \mathrm{SO}_{4}$. Gebumden 15,5 ccm $\frac{\mathrm{n}}{10,2803} \mathrm{H}_{2} \mathrm{SO}_{4}=0,0211 \mathrm{~g} \mathrm{~N}$. In 1,52 $\mathrm{g}$ Kot 0,0322 $\mathrm{g} \mathbf{N}$. 15. Oktober 1907. $25 \mathrm{ccm} \frac{\mathrm{n}}{10,2808} \mathrm{H}_{2} \mathrm{SO}_{4}$ vorgelegt.

Kaninchen Nr. 19. $1 \mathrm{~g}$ Kot durch $\mathrm{H}_{2} \mathrm{SO}_{4}$ zerstört. Titriert 17,2 cem $\frac{\mathrm{n}}{\mathrm{x}} \mathrm{NaOH}=$ $13,7 \mathrm{ccm} \frac{\mathrm{n}}{10,2803} \mathrm{H}_{2} \mathrm{SO}_{4}$. Gebunden $11,3 \mathrm{ccm} \frac{\mathrm{n}}{10,2808} \mathrm{H}_{2} \mathrm{SO}_{4}=0,0154 \mathrm{~g} \mathrm{~N}$. In $1,7 \mathrm{~g}$ Kot $0,0269 \mathrm{~g} \mathbf{N}$.

Schwefelbestimmungen in 5 cem Urin.

7. Oktober 1907.

Kaninchen Nr. 19. a) $\left.0,01663 \mathrm{~g} \mathrm{BaSO}_{4}=0,0023 \mathrm{~g} \mathrm{~S}\right\}$

b) $\left.0,01598 \mathrm{~g} \mathrm{BaSO}_{4}=0,0022 \mathrm{~g} \mathrm{~S}\right\}$ Nittel $0,00225 \mathrm{~g} \mathrm{~S}$. In $600 \mathrm{ccm}$ Urin + Spülwasser $0,2700 \mathrm{~g} \mathrm{~S}$.

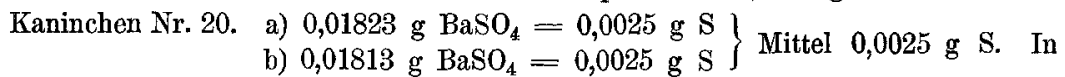
$490 \mathrm{ccm}$ Urin + Spülwasser $0,2450 \mathrm{~g} \mathrm{~S}$.

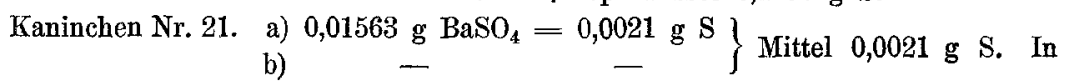
$500 \mathrm{ccm}$ Urin + Spülwasser $0,2100 \mathrm{~g} \mathrm{~S}$. 


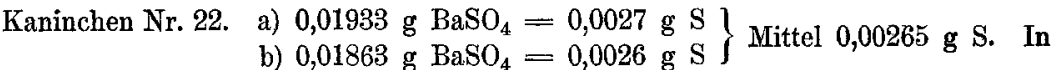
$565 \mathrm{ccm}$ Urin + Spülwasser $0,2994 \mathrm{~g} \mathrm{~S}$. .

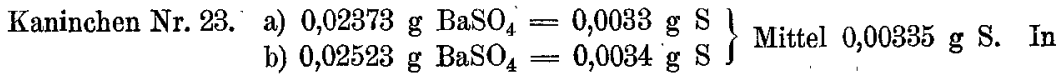
$415 \mathrm{ccm}$ Urin + Spülwasser $0,2780 \mathrm{~g} \mathrm{~S}$.

9. Oktober 1907.

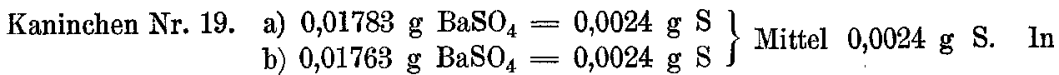
$465 \mathrm{ccm}$ Urin + Spülwasser $0,2232 \mathrm{~g} \mathrm{~S}$.

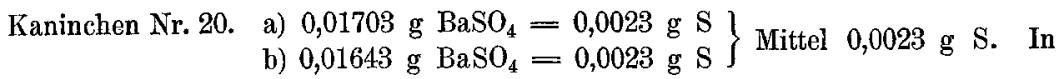
$415 \mathrm{ccm}$ Urin + Spülwasser $0,1909 \mathrm{~g} \mathrm{~S}$.

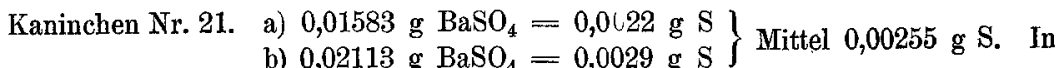
$455 \mathrm{ccm}$ Urin + Spülwasser $0,2830 \mathrm{~g} \mathrm{~S}$.

Kaninchen Nr. 22. a) $0,01963 \mathrm{~g} \mathrm{BaSO}_{4}=0,0027 \mathrm{~g} \mathrm{~S} \cdot \mathrm{gittel} 0,00265 \mathrm{~g}$ S. In $460 \mathrm{cem}$ Urin + Spülwasser $0,2438 \mathrm{~g} \mathrm{~S}$.

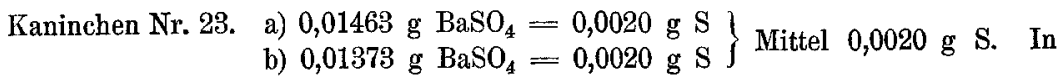
$545 \mathrm{ccm}$ Urin + Spülwasser $0,2180 \mathrm{~g} \mathrm{~S}$.

11. Oktober 1907.

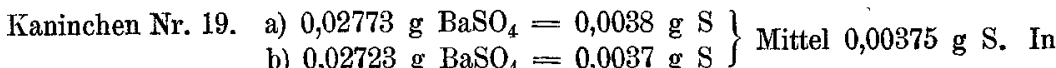
$250 \mathrm{ccm}$ Urin + Spülwasser $0,1875 \mathrm{~g} \mathrm{~S}$."

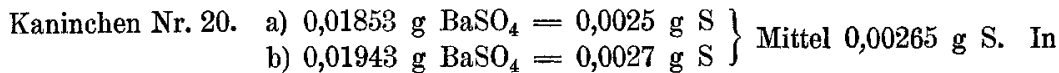
$160 \mathrm{ccm}$ Urin + Spülwasser $0,0848 \mathrm{~g} \mathrm{~S}$.

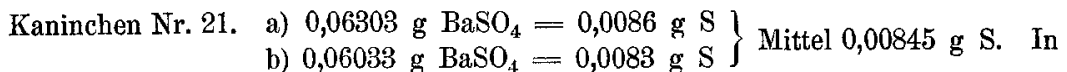
$205 \mathrm{ccm}$ Urin + Spülwasser 0,3464 g S.

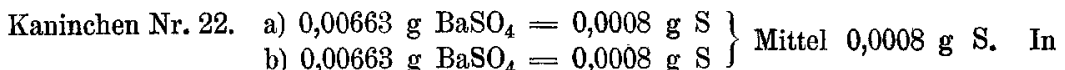
$270 \mathrm{ccm}$ Urin + Spülwasser $0,0432 \mathrm{~g} \mathrm{~S}$.

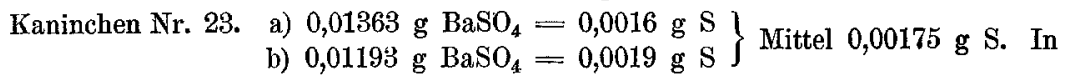
$185 \mathrm{ccm}$ Urin + Spülwasser $0,0647 \mathrm{~g} \mathrm{~S}$.

13. Oktober 1907.

Kaninchen Nr. 19. a) $0,01363 \mathrm{~g} \mathrm{BaSO}_{4}=0,0019 \mathrm{~g} \mathrm{~S}$

b) $\left.0,01233 \mathrm{~g} \mathrm{BaSO}_{4}=0,0017 \mathrm{~g} \mathrm{~S}\right\}$ Mittel $0,0018 \mathrm{~g} \mathrm{~S}$. In $190 \mathrm{ccm}$ Urin + Spülwasser $0,0684 \mathrm{~g} \mathrm{~S}$.

Kaninchen Nr. 20 . a) 0,01813 g $\left.\mathrm{BaSO}_{4}=0,0025 \mathrm{~g} \mathrm{~S}\right\}$ Mittel 0,0025 g S. In 165 ccm Urin + Spülwasser $0,0825 \mathrm{~g} \mathrm{~S}$. 
Kaninchen Nr. 21. a) $\left.0,01423 \mathrm{~g} \mathrm{BaSO}_{4}=0,0020 \mathrm{~g} \mathrm{~S}\right)$

b) $\left.0,01623 \mathrm{~g} \mathrm{BaSO}_{4}=0,0022 \mathrm{~g} \mathrm{~S}\right\}$ Mittel $0,0021 \mathrm{~g} \mathrm{~S}$. In

$185 \mathrm{ccm}$ Urin + Spülwasser $0,0777 \mathrm{~g} \mathrm{~S}$.

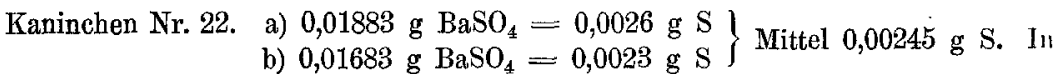

$180 \mathrm{ccm}$ Urin + Spülwasser $0,0825 \mathrm{~g} \mathrm{~S}$.

Kaninchen Nr. 23. a) 0,02239 g $\left.\begin{array}{l}\mathrm{BaOS}_{4}=0,0030 \mathrm{~g} \mathrm{~S} \\ \text { b) } 0,02443 \mathrm{~g} \mathrm{BaSO}_{4}=0,0033 \mathrm{~g} \mathrm{~S}\end{array}\right\}$ Mittel $0,00315 \mathrm{~g} \mathrm{~S}$. In

$195 \mathrm{ccm}$ Urin + Spülwasser $0,1228 \mathrm{~g} \mathrm{~S}$.

15. Oktober 1907.

Kaninchen Nr. 19. a) 0,01303 g $\left.\mathrm{BaSO}_{4}=0,0018 \mathrm{~g} \cdot \mathrm{S}\right\}$

b) $\left.0,01503 \mathrm{~g} \mathrm{BaSO}_{4}=0,0021 \mathrm{~g} \mathrm{~S}\right\}$ Mittel $0,00195 \mathrm{~g} \mathrm{~S}$. In

$140 \mathrm{ccm}$ Urin + Spülwasser $0,0546 \mathrm{~g} \mathrm{~S}$.

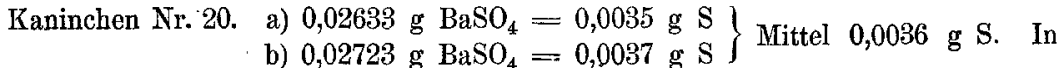

$130 \mathrm{ccm}$ Urin + Spülwasser 0,0936 g S.

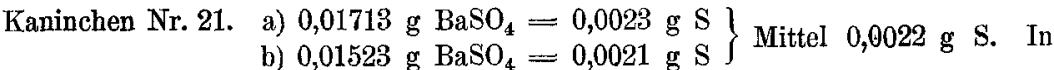

$145 \mathrm{ccm}$ Urin + Spülwasser $0,0638 \mathrm{~g} \mathrm{~S}$.

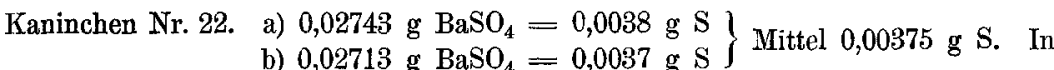

$145 \mathrm{ccm}$ Urin + Spülwasser 0,1087. $\mathrm{g} \mathrm{S}$.

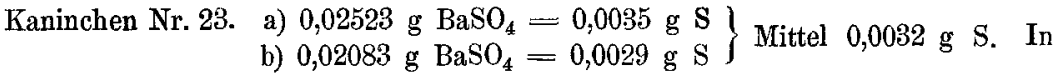

$150 \mathrm{ccm}$ Urin + Spülwasser 0,0960 g S.

17. Oktober 1907.

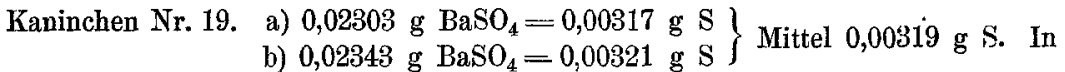

$155 \mathrm{ccm}$ Urin + Spülwasser $0,0989 \mathrm{~g} \mathrm{~S}$.

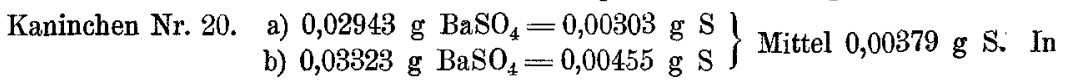

$110 \mathrm{ccm}$ Urin + Spülwasser $0,0834 \mathrm{~g} \cdot \mathrm{S}$.

Kaninchen Nr. 21. a) 0,02023 g $\left.\mathrm{BaSO}_{4}=0,00277 \mathrm{~g} \mathrm{~S}\right\}$ Mittel $0,00277 \mathrm{~g}$ S. In

$145 \mathrm{ccm}$ Urin + Spülwasser $0,0803 \mathrm{~g} \mathrm{~S}$.

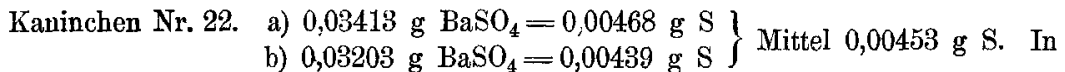

$180 \mathrm{ccm}$ Urin + Spülwasser $0,16308 \mathrm{~g} \mathrm{~S}$.

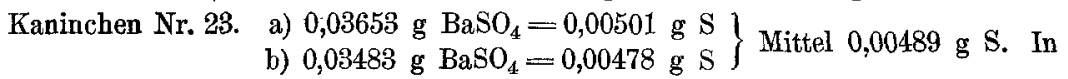

$160 \mathrm{ccm}$ Urin + Spülwasser $0,1565 \mathrm{~g} \mathrm{~S}$.

19. Oktober 1907.

$\left.\begin{array}{l}\text { Kaninchen Nr. 19. a) } 0,05403 \mathrm{~g} \mathrm{BaSO}_{4}=0,00741 \mathrm{~g} \mathrm{~s} \\ \text { b) } 0,05433 \mathrm{~g} \mathrm{BaSO}_{4}=0,00745 \mathrm{~g} \mathrm{~S}\end{array}\right\}$ Mittel 0,00743 g S. In $155 \mathrm{ccm}$ Urin + Spülwasser $0,2303 \mathrm{~g} \mathrm{~S}$. 


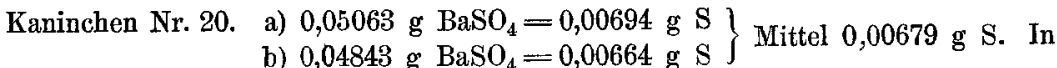
$140 \mathrm{ccm}$ Urin + Spülwassser $0,1901 \mathrm{~g} \mathrm{~S}$.

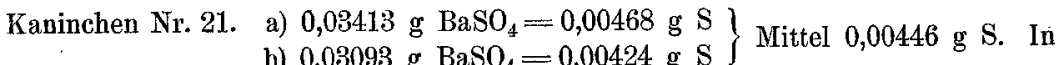

b) $0,00093 \mathrm{~g} \mathrm{BaSO}_{4}=0,00424 \mathrm{~g} \mathrm{~S}$. Spülwasser $0,1026 \mathrm{~g} \mathrm{~S}$.

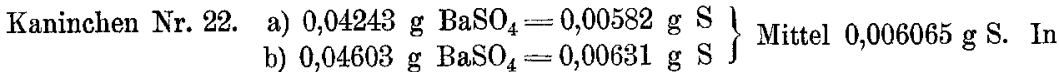
$210 \mathrm{ccm}$ Urin + Spülwasser 0,2545 g S.

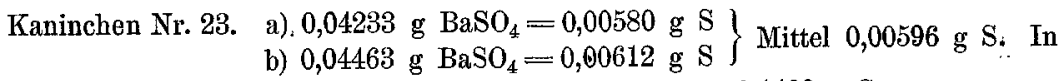
$125 \mathrm{ccm}$ Urin + Spülwasser $0,1490 \mathrm{~g}$. S.

21. Oktober 1907.

Kaninchen Nr. 19 . a) $\left.0,02843 \mathrm{~g} \mathrm{BaSO}_{4}=0,00390 \mathrm{~g} \mathrm{~S}\right\}$ Mittel $0,003835 \mathrm{~g} \mathrm{~S}$. In b) $\begin{array}{r}0,02753 \mathrm{~g} \mathrm{BaSO}=0,00377 \mathrm{~g} \mathrm{~S} \text { / Mittel } 0,0038 \mathrm{~g} \mathrm{~S} . \\ 125 \mathrm{ccm} \text {. Urin + Spülwasser } 0,0959 \mathrm{~g} \mathrm{~S}\end{array}$ b) $0,06683 \mathrm{~g} \mathrm{BaSO}=0,00916 \mathrm{~g} \mathrm{~S}$ Mittel 0,0090

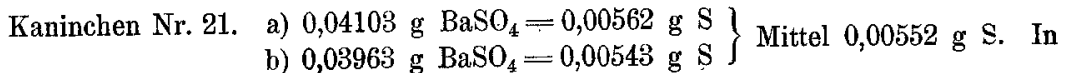
$125 \mathrm{ccm}$ Urin + Spülwasser $0,1380 \mathrm{~g} \mathrm{~S}$.

23. Oktober 1907.

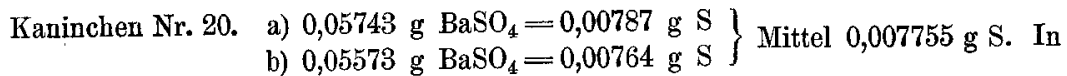

$150 \mathrm{ccm}$ Urin + Spülwasser $0,2326 \mathrm{~g} \mathrm{~S}$.

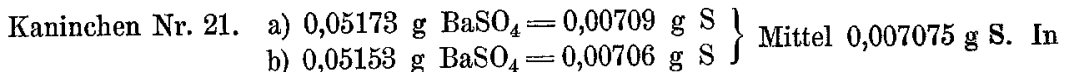
$165 \mathrm{ccm}$ Urin + Spülwasser $0,2335 \mathrm{~g} \mathrm{~S}$.

Phosphorbestimmungen in 25 cem Urin.

7. Oktober 1907.

Kaninchen Nr. 19. Titriert a) 2,9 Y. Mittel 2,95 ccm Urannitrat $=0,0065 \mathrm{~g} \mathrm{P}$. In b) 3,0$\} \quad 600 \mathrm{ccm}$ Urin + Spülwasser $0,2058 \mathrm{~g} \mathbf{P}$.

Kaninchen Nr. 20. Titriert a) 6,2 \} Mittel 6,25 ccm Urannitrat $=0,0139$ g P. In

b) 6,3$\} \quad 490 \mathrm{ccm}$ Urin + Spülwasser $0,2724 \mathrm{~g} \mathrm{P}$.

Kaninchen Nr. 21. Titriert a) 3,4 ) Mittel $3,4 \mathrm{ccm}$ Urannitrat $=0,0076 \mathrm{~g}$ P. In

b) 3,4$\} 500 \mathrm{ccm}$ Urin + Spülwasser $0,1560 \mathrm{~g} \mathrm{P}$.

Kaninchen Nr. 22. Titriert a) 1,4 $\}$. Mittel 1,4 ccm Urannitrat $=0,0031 \mathrm{~g} \mathrm{P}$. In

b) 1,4$\} \quad 565 \mathrm{ccm}$ Urin + Spülwasser $0,071 \mathrm{~g} \mathrm{P}$.

Kaninchen Nr. 23. Titriert a) 5,6 Mittel $5,55 \mathrm{ccm}$ Urannitrat $=0,0124 \mathrm{~g} \mathrm{P}$. In

b) 5,5$\} \quad 415 \mathrm{ccm}$ Urin + Spülwasser $0,2058 \mathrm{~g} \mathrm{P}$.

9. Oktober 1907.

Kaninchen Nr. 19. Titriert a) 4,8 Mittel $4,85 \mathrm{ccm}$ Urannitrat $=0,0108 \mathrm{~g}$ P. In

b) 4,9$\} \quad 465 \mathrm{ccm}$ Urin + Spülwasser $0,2009 \mathrm{~g} \mathrm{P}$.

Kaninchen Nr. 20. Titriert a) 6,3 $\}$ Mittel $6,25 \mathrm{ccm}$ Urannitrat $=0,0139 \mathrm{~g} \mathrm{P}$. In

b) 6,2$\} \quad 415 \mathrm{ccm}$ Urin + Spülwasser $0,2307 \mathrm{~g} \mathrm{P}$. 
Der Einfluss des Alkohols am Hungertier auf Lebensdauer etc.

Kaninchen Nr. 21. Titriert a) 4,9 | Mittel $5,0 \mathrm{ccm}$ Urannitrat $=0,0111 \mathrm{~g}$ P. In

b) 5,1$\} \quad 455 \mathrm{ccm}$ Urin + Spülwasser $0,2020 \mathrm{~g} \mathrm{P}$.

Kaninchen Nr. 22. Titriert a) 3,8 \Mittel 3,7 ccm Urannitrat $=0,0082$ g P. In

b) 3,6$\} \quad 460 \mathrm{ccm}$ Urin + Spülwasser $0,1509 \mathrm{~g} \mathrm{P}$.

Kaninchen Nr. 23. Titriert a) 3,3 . Mittel 3,4 ccm Urannitrat $=0,0076$ g P. In

b) 3,5$\} \quad 545 \mathrm{ccm}$ Urin + Spülwasser $0,1657 \mathrm{~g} \mathrm{P}$.

11. Oktober 1907.

Kaninchen Nr. 19. Titriert a) 16,1 Mittel $16,2 \mathrm{ccm}$ Urannitrat $=0,0361 \mathrm{~g}$ P. In

b) 16,3$\} \quad 250 \mathrm{ccm}$ Urin + Spülwasser $0,3610 \mathrm{~g} \mathrm{P}$.

Kaninchen Nr. 20. Titriert a) 17,4 Mittel 17,4 ccm Urannitrat $=0,0387$ g P. In

b) 17,4$\} 160 \mathrm{ccm}$ Urin + Spülwasser $0,2477 \mathrm{~g} \mathrm{P}$.

Kaninchen Nr. 21. Titriert a) 16,0$\}$ Mittel $16,05 \mathrm{ccm}$ Urannitrat $=0,0357 \mathrm{~g}$ P. In

b) 16,1$\} 205 \mathrm{ccm}$ Urin + Spülwasser $0,2927 \mathrm{~g} \mathrm{P}$.

Kaninchen Nr. 22. Titriert a) 6,2 Mittel 6,1 ccm Urannitrat $=0,0136 \mathrm{~g} \mathrm{P}$. In

b) $6,0 \mathrm{~J} 270 \mathrm{ccm}$ Urin + Spülwasser $0,1469 \mathrm{~g} \mathrm{P}$.

Kaninchen Nr. 23. Titriert a) 11,7$\}$ Mittel $11,8 \mathrm{ccm}$ Lrannitrat $=0,0263 \mathrm{~g}$ P. In

b) 11,9$\} 185 \mathrm{ccm}$ Urin + Spülwasser $0,1946 \mathrm{~g} \mathrm{P}$.

Phosphorbestimmungen in 10 cem Urin.

13. Oktober 1907.

Kaninchen Nr. 19. Titriert a) 4,8 Mittel 4,8 ccm Urannitrat $=0,0110$ g P. In b) 4,8$\} 190 \mathrm{ccm}$ Urin + Spülwasser $0,2033 \mathrm{~g} \mathrm{P}$.

Kaninchen Nr. 20. Titriert a) 6,2 \ittel 6,2 ccm Urannitrat $=0,0138$ g P. In

b) $6,2 \mathrm{~J} 165 \mathrm{cem}$ Úrin + Spülwasser $0,2277 \mathrm{~g} \mathrm{P}$.

Kaninchen Nr. 21. Titriert a) 5,0 Mittel 5,05 ccm Urannitrat $=0,0112 \mathrm{~g} \mathrm{P}$. In

b) 5,1$\} 185 \mathrm{ccm}$ Urin + Spülwasser $0,2072 \mathrm{~g} \mathrm{P}$.

Kaninchen Nr. 22. Titriert a) 8,1 $\}$ Mittel $8,1 \mathrm{ccm}$ Urannitrat $=0,0180 \mathrm{~g} \mathrm{P}$. In

b) 8,1$\} 180 \mathrm{ccm}$ Urin + Spülwasser $0,3240 \mathrm{~g} \mathbf{P}$.

Kaninchen Nr. 28. Titriert a) 6,5 Mittel 6,55 ccm Urannitrat $=0,0146 \mathrm{~g}$ P. In

b) 6,6$\} 195 \mathrm{ccm}$ Urin + Spülwasser $0,2847 \mathrm{~g} \mathrm{P}$.

15. Oktober 1907.

Kaninchen Nr. 19. Titriert a) 4,9 $\}$ Mittel 4,9 $\mathrm{cm}$ Urannitrat $=0,0109 \mathrm{~g}$ P. In

b) 4,9$\} 140 \mathrm{ccm}$ Urin + Spülwasser $0,1526 \mathrm{~g}$ P.

Kaninchen Nr. 20. Titriert a) 5,1 Mittel $5,15 \mathrm{ccm}$ Urannitrat $=0,0115 \mathrm{~g}$ P. In

b) 5,2$\} 130 \mathrm{ccm}$ Urin + Spülwasser $0,1495 \mathrm{~g} \mathrm{P}$.

Kaninchen Nr. 21. Titriert a) 4,8 Mittel $4,9 \mathrm{ccm}$ Urannitrat $=0,0109 \mathrm{~g}$ P. In

b) 5,0$\} 145 \mathrm{ccm}$ Urin + Spülwasser $0,1581 \mathrm{~g} \mathrm{P}$.

Kaninchen Nr. 22. Titriert a) 5,9 Mittel $5,75 \mathrm{ccm}$ Urannitrat $=0,0128 \mathrm{~g} \mathrm{P}$. In

b) $5,6 \mathrm{f} 145 \mathrm{ccm}$ Urin + Spülwasser $0,1856 \mathrm{~g} \mathrm{P}$.

Kaninchen Nr. 23. Titriert a) 6,3 | Mittel 6,2 $\mathrm{ccm}$ Urannitrat $=0,0138 \mathrm{~g}$ P. In

17. Oktober 1907.

b) 6,1$\} \quad 150 \mathrm{ccm}$ Urin + Spülwasser $0,2070 \mathrm{~g} \mathrm{P}$.

Kaninchen Nr. 19. Titriert a) 5,1 Mittel 5,2 ccm Urannitrat $=0,0116 \mathrm{~g}$ P. In

b) 5,3$\} \quad 155 \mathrm{ccm}$ Urin + Spülwasser $0,1798 \mathrm{~g} \mathrm{P}$.

Kaninchen Nr. 20. Titriert a) 5,1 Mittel $5,1 \mathrm{ccm}$ Urannitrat $=0,0114 \mathrm{~g} \mathrm{P}$. In

b) 5,1$\} 110 \mathrm{ccm}$ Urin = Spülwasser $0,1254 \mathrm{~g} \mathrm{P}$. 
356 Martin Kochmann und W. Hall: Der Einfluss des Atrohols ete.

Kaninchen' Nr. 21. Titriert a) 5,7 'Mittel $5,65 \mathrm{ccm}$ Urannitrat $=0,0126 \mathrm{~g}$ P. In b) $5,6 \mathrm{f} 145 \mathrm{ccm}$ Urin + Spülwasser $0,1827 \mathrm{~g} \mathrm{P}$.

Kaninchen Nr. 22. Titriert a) 4,6 Mittel $4,6 \mathrm{~cm}$. Urannitrat $=0,0102 \mathrm{~g}$ P. In b) 4,6 . 180 cém Urin + Spülwasser $0,1836 \mathrm{~g} \mathrm{P}$.

Kaninchen Nr. 28. Titriert a) 6,2 \} Mittel $6,3 \mathrm{~cm}$ Urannitrat $=0,0140 \mathrm{~g} \mathrm{P.AIn}$ b) 6,4$\} \quad 160 \mathrm{lcm} \cdot$ Jrin + Spülwasser $0,2240 \mathrm{~g} \mathrm{P}$.

19. Oktober 1907.

Kaninchen Nr. 19. Titriert a) 6,0$\}$ Mittel $5,9 \mathrm{ccm}$ Urannitrat $=0,0126 \mathrm{~g}$. In b) 5,8$\} \quad 155 \mathrm{ccm}$ Urin + Spülwasser $0,1953 . \mathrm{g} \mathrm{P}$.

Kaninchen Nr. 20. Titriert a) 8,0 \} Mittel $8,05 \mathrm{ccm}$ Urannitrat $=0,0172 \mathrm{~g}$ P." In

b) 8,1$\}, \quad 140 \mathrm{ccm}$ Urin + Spülwasser. 0,2408.g P.

Kaninchen Nr. 21. Titriert a) 6,1 Mittel $6,1 \mathrm{~cm}$ Urannitrat $=0,0130 \mathrm{~g}$. In b) 6,1$\} \quad 145 \mathrm{~cm}$ Urin + Spülwasser $0,1495 \mathrm{~g} P$.

Kaninchen Nr. 22. Titriert a) 7,0 | Mittel $7,0 \mathrm{~cm}$ Urannitrat $=0,0149$ g P. In b) $7,0 \mathrm{~J}^{-\infty} 210 \mathrm{~cm}$ Urin + Spülwasser $0,3129 \mathrm{~g} \mathrm{P}$.

Kaninchen Nr. 23. Titriert a) 6,9 , Mittel $6,9 \mathrm{ccm}$ Urannitrat $=0,0147 \mathrm{~g}$ P. In b) 6,9$\} \quad 125 \mathrm{ccm}$ Urin + Spülwasser $0,1836 \mathrm{~g} \mathrm{P}$

21. Oktober 1907.

Kaninchen Nr. 19. Titriert a) 4,4 Mittel 4,4 ccm Urannitrat $=0,0094 \mathrm{~g}$ P. In b) 4,4$\}$. $\quad 125 \mathrm{ccm}$ Urin + Spülwasser $0,1175 \cdot \mathrm{g} \mathrm{P}$.

Kaninchen Nr. 20. Titriert a) 10,0$\}$ Mittel $10,1 \mathrm{ccm}$ Urannitrat $=0,0216 \mathrm{~g}$ P. In b) 10,2$\}: 140 \mathrm{ccm}$ - Urin + Spülwasser $0,3024 \mathrm{~g} \mathrm{P}$.

Kaninchen Nr. 21. Titriert a) 4,9: Mittel $5,05 \mathrm{ccm}$ Urannitrat $=0,0108 \mathrm{~g} \cdot \mathrm{P}$. In b) 5,2 i 125 cdm Urin + Spülwasser $0,1350 \mathrm{~g} \mathrm{P}$.

23. Oktober 1908 .

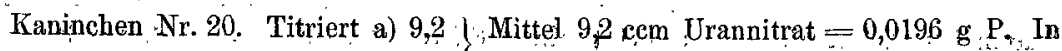
b) 9,2$\}$ : $150 \mathrm{xcm}$. Urin + Spülwasser $0,2940 \mathrm{~g} \mathrm{P}$.

Kaninchen Nr. 21. Titriert a) 5,5$\}$ Mittel 5,6 cen Urannitrat $=0,0120$ g P. In

b) $5,7 \int_{-,} \quad 165$ cem, Urin + Spülwasser $0,1980 \mathrm{~g} \mathrm{P}$. 